Faculty of Engineering of the University of Porto

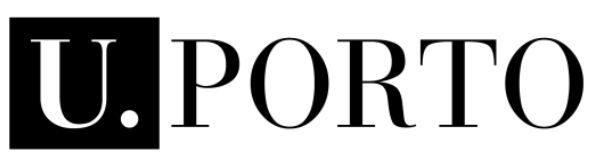

FEUP FACULDADE DE ENGENHARIA
UNIVERSIDADE DO PORTO

\title{
Quantitative Assessment of Central Serous Chorioretinopathy in Angiographic Sequences of Retinal Images
}

Carlos Alexandre Nunes Ferreira

MASTER DISSERTATION

Master in Bioengineering

Major in Biomedical Engineering

Supervisor: Prof. Dr. Ana Maria Mendonça

Co-supervisor: Prof. Dr. Jorge Alves da Silva 
(c) Carlos Alexandre Nunes Ferreira, 2017 


\section{Abstract}

Central serous chorioretinopathy is a retinal disease in which there is a leakage of fluid into the subretinal space through a retinal pigment epithelium lesion that may cause a serous detachment of the neurosensory retina. Fluorescein angiography images allow the identification of these leaks. In this type of images, the lesions and the blood vessels appear bright and the remaining anatomical structures of the retina appear dark. The area related to the leakage increases throughout the angiographic sequences and, in general, the leakage can only be visualized completely in the later phases of the exam.

In this work, computational methods of image processing and image analysis are used for the detection, characterization, and determination of the size progression of dye leaks along the angiographic sequences. These methods were integrated into a computer-aided diagnosis tool.

To the best of our knowledge, a computer-aided diagnosis tool that allows the automatic characterization of leakage of central serous chorioretinopathy in fluorescein angiography images is not described in the literature. The central serous chorioretinopathy leakage segmentation problem is similar to leakage diseases like the diabetic macular edema, the macular retinopathy or the choroidal neovascularization. The segmentation methods for these three diseases are divided into three main categories: comparative by subtraction of images, comparative with classification and saliency detection. The main challenges to characterize the leakage are the difference in luminosity between images of the angiography sequence, the similar pixel intensities of the leaks and the vessels, and the late staining of the optic disc.

The comparative methods by subtraction of images are used for the automatic characterization of the leakage of central serous chorioretinopathy in angiographic sequences because they use temporal information. As the leakage area grows during examination, temporal information helps with identification. Furthermore, algorithm steps were introduced to reduce the influence of anatomical elements (such as the background, vessels, and optic disc) in localization and segmentation of leaks. The steps are frame selection, image denoising, image registration, vessel segmentation, candidate selection in early frames, vessel inpainting, 
optic disc detection, intensity normalization, background removal, image subtraction and leakage segmentation. The leakage segmentation is processed in three phases. First, candidates are selected in the subtraction image with the Otsu method. Second, the region growing algorithm is applied to the candidate regions to segment leaks in the last frame of the sequence. Finally, the segmentation of the leaks in the remaining frames of the sequence is achieved through an algorithm of active contours, i.e., in each frame, the leaks are segmented using as input the contours of the segmented leaks in the frame immediately later. As all the leaks are segmented in all frames of the sequence, the size progression of the leak over time is also analyzed.

The results were compared with the available manual annotations. They are adequate for the training set with $100 \%$ detection and mean Jaccard index of $0.72 \pm 0.17$. In the test set, the results are also satisfactory. The manual annotations of the test set corresponded to the most deviant cases and even so a mean Jaccard index of $0.69 \pm 0.12$ was achieved. The detection only failed for the smaller leakages and when there are leakages with very different intensity means in the same image. The segmentation problems happen when the leaks have prolongated angiographic haze regions.

Keywords: central serous chorioretinopathy, fluorescein angiography, angiographic sequence, image subtraction, leakage segmentation, leakage grow. 


\section{Resumo}

A corioretinopatia central serosa é uma doença retiniana caracterizada pelo desprendimento da retina neurosensorial da camada do epitélio pigmentado da retina, com consequente acumulação de líquido no espaço subretiniano vindo da coroide. Estas lesões podem ser detetadas em imagens de exames de angiografia fluoresceínica nas quais as lesões aparecem claras, como os vasos sanguíneos, e as restantes estruturas anatómicas da retina aparecem escuras. A área referente ao derrame aumenta ao longo das sequências angiográficas pelo que só em fases tardias do exame é possível visualizá-lo totalmente.

Neste trabalho, métodos computacionais de processamento e análise de imagem foram usados para detetar, caracterizar e analisar a progressão do crescimento de derrames ao longo das sequências angiográficas. Estes métodos foram integrados numa ferramenta de apoio ao diagnóstico.

Não foram encontrados métodos computacionais de apoio ao diagnóstico que permitam a caraterização automática do derrame da corioretinopatia central serosa em imagens de angiografia fluoresceínica. No entanto, o problema de segmentação é similar a outras doenças que originam derrames como o edema macular diabético, a retinopatia macular ou a neovascularização coroidal. Os métodos de segmentação nestas doenças dividem-se em três principais grupos: comparativos por subtração de imagens, comparativos com classificação e de deteção de saliência. Os principais desafios para caraterizar o derrame são as diferenças de iluminação nas imagens da sequência angiográfica, as intensidades dos píxeis do derrame serem semelhantes às dos vasos e a coloração tardia do disco ótico.

Os métodos comparativos por subtração de imagens foram escolhidos para a caraterização automática dos derrames da corioretinopatia central serosa em imagens de sequências angiográficas pois usam informação temporal. Como o derrame tende a crescer durante o exame, a informação temporal pode auxiliar na sua identificação. Além do mais são introduzidos passos que permitem a redução da influência dos elementos anatómicos (como o fundo, vasos e disco ótico) na localização e segmentação dos derrames. Os passos introduzidos foram a seleção de imagens, a redução de ruído, o alinhamento, a segmentação dos vasos, a 
deteção de candidatos nas imagens iniciais, a substituição das intensidades dos vasos por valores das suas vizinhanças, a deteção do disco ótico, a normalização da intensidade, a remoção do fundo, a subtração de imagens e por fim, a segmentação dos derrames. A segmentação dos derrames é realizada em três fases. Em primeiro lugar, são determinados candidatos na imagem diferença através do método Otsu. Em segundo lugar, um algoritmo de crescimento de região é aplicado para que seja possível obter a segmentação da lesão no último frame da sequência. Por fim, a segmentação dos restantes derrames da sequência é determinada utilizando um algoritmo de contornos ativos, ou seja, para cada frame, a área do derrame é determinada utilizando na primeira iteração do algoritmo o contorno do derrame determinado no frame imediatamente mais tardio. Como todos os derrames são segmentados em todos os frames da sequência, é possível analisar a progressão de crescimento do derrame ao longo do tempo.

Os resultados foram comparados com marcações manuais. Para o conjunto de treino, foram bastante satisfatórios já que houve uma deteção de 100\% e uma média do índice Jaccard de $0,72 \pm 0,17$. Para o conjunto de teste, os resultados também são aceitáveis. As marcações manuais disponíveis apenas correspondiam aos casos mais complicados, mas mesmo assim a média do índice Jaccard foi de 0,69 $\pm 0,12$. Além do mais, a deteção apenas falhou para casos de pequenos derrames, principalmente em situações em que os derrames tinham diferentes intensidades médias. Já os piores resultados de segmentação verificam-se quando os derrames têm uma região de haze angiográfico prolongado.

Palavras-chave: corioretinopatia central serosa, angiografia fluoresceínica, sequência angiográfica, subtração de imagens, segmentação do derrame, crescimento do derrame. 


\section{Agradecimentos}

O trabalho de dissertação é o culminar de cinco anos de emoções muito distintas, umas vezes positivas, outras vezes negativas. No entanto, as coisas boas acabaram ainda assim por se sobressair e tenho de agradecer a um conjunto de pessoas que foram muito importantes neste meu percurso.

Em primeiro lugar, lembro-me dos meus pais. Nunca tiveram oportunidade de concluir os estudos e sempre incentivaram para que eu, o filho mais velho, acabasse com sucesso esta etapa. Mesmo com as dificuldades económicas que passámos nos últimos tempos, nunca me faltaram com nada, abdicando por vezes de regalias pessoais para que eu pudesse ter as melhores condições de estudo possíveis. Os meus avós são, por seu lado, uma grande fonte de carinho. Parece que dizem sempre as palavras certas nos momentos adequados e de facto isto é muito revitalizante nos dias que me sinto mais em baixo. 0 meu irmão também é uma fonte de alegria. Arranja sempre brincadeiras para se entreter, ele diverte-se e diverte-nos. Por fim, uma palavra de ressalva ao meu tio Dinis, tio Jorge, tia Alice e prima Sandra. São incansáveis e sei que sempre poderei contar com eles.

Uma palavra de apreço também tem de ser dada aos meus amigos de Lamego, apesar de termos tomado rumos diferentes sempre nos vamos encontrado e apoiando nos momentos mais críticos. Mas uma nova família também me acolheu no Porto, Metal\&Bio: um grupo de estudantes irreverentes, diferentes e que sabe conciliar os diversos momentos da vida académica. Foi um grande orgulho ter contribuído para o crescimento deste grupo.

Ficam também os meus agradecimentos ao José Alves por ter sido um fiel parceiro de grupo, ao Carlos Guimarães por ter sido um fiel companheiro de diversão e ao João Araújo por ter sido um fiel companheiro de aleatoriedade. É formidável verificar que este grupo mais restrito de amigos se manteve desde o primeiro ano.

A vida académica também é uma vida descoberta. Descobri que gosto muito da gestão de equipas. Foi um gosto comandar o NEB e o EMBS ao longo destes dois anos.

Por fim, uma palavra de apreço às pessoas que me conduziram nesta dissertação. Obrigado professora Ana Maria Mendonça e professor Jorge Silva. Nem sempre me conseguia explicar da 
melhor maneira, mas sempre fui ouvido e sempre fui ajudado em todas as dificuldades que me fui deparando ao longo do meu trabalho de dissertação. De igual forma a médica Susana Penas foi essencial em todo o processo, dado que forneceu as imagens de angiografia e as marcações manuais, mostrando-ainda disponível para clarificar qualquer dúvida de interpretação que eu tivesse dos resultados. 
"Caminha devagar, vive intensamente, o melhor aparece de repente."

Carlos Alexandre Nunes Ferreira 


\section{Index}

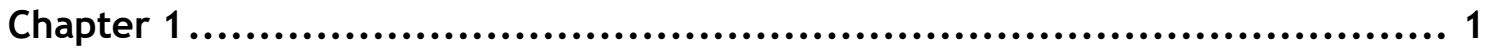

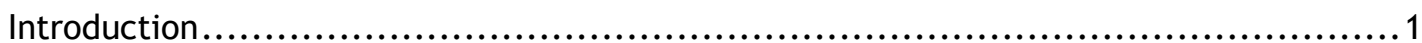

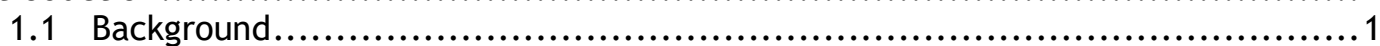

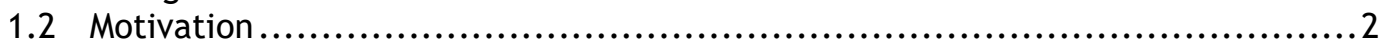

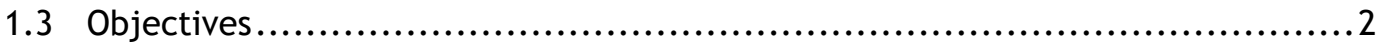

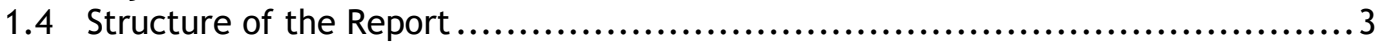

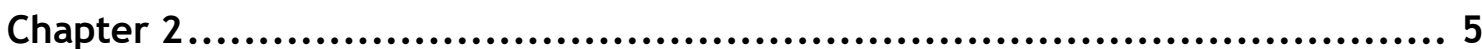

Central Serous Chorioretinopathy....................................................... 5

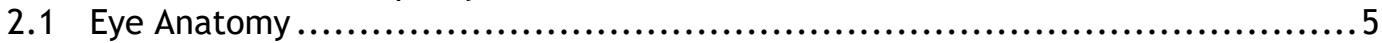

2.2 Central Serous Chorioretinopathy Description .................................

2.3 Imaging Modalities for CSC Diagnosis......................................... 8

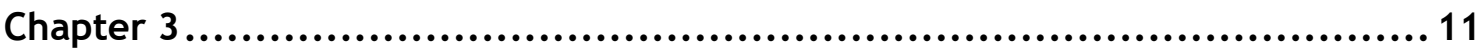

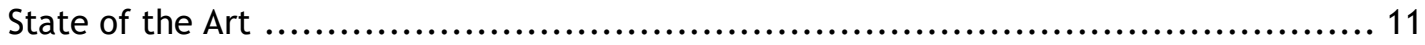

3.1 Challenges................................................................... 11

3.2 Leakage Assessment: a Literature Review ................................. 13

3.2.1 Frame Selection ..................................................... 15

3.2.2 Image Registration ................................................... 16

3.2.3 Vessel Segmentation ................................................. 17

3.2.4 Optic Disc Segmentation........................................... 18

3.2.5 Modeling the Background Intensity ....................................... 19

3.2.6 Saliency Detection ..................................................... 19

3.2.7 Segmentation in Image Subtraction Approaches ........................ 20

3.2.8 Segmentation in Classification Approaches ................................ 20

3.2.9 Segmentation in Saliency Approaches ................................. 21

3.2.10 Evaluation ............................................................ 21

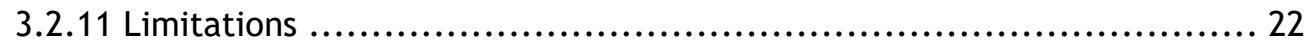

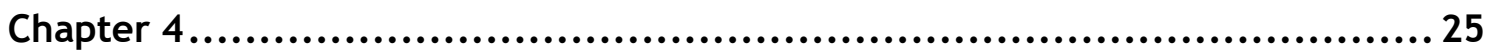

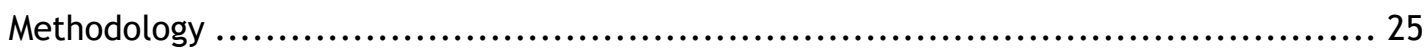

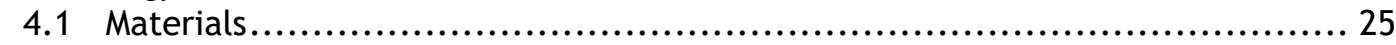

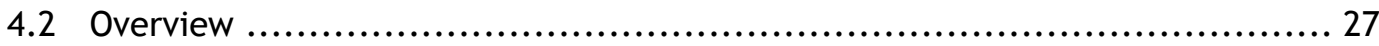

4.3 Frame Selection .................................................................. 30

4.4 Time Detection ....................................................................... 31

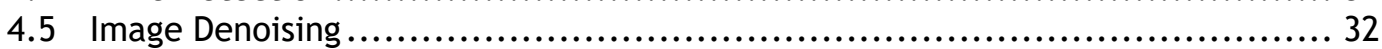

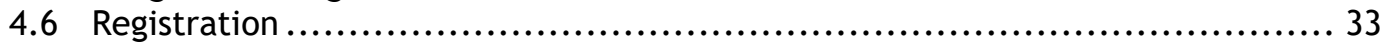

4.7 Vessel Segmentation ........................................................... 36

4.8 Candidate Selection in the Early Frames ................................................... 38

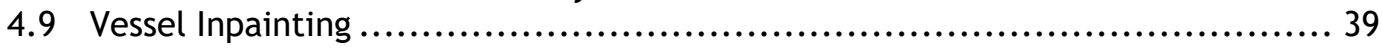


4.10 Optic Disc Detection ..................................................... 41

4.11 Intensity Normalization ................................................ 45

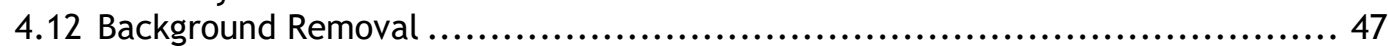

4.13 Image Subtraction ........................................................ 48

4.14 Leakage Segmentation ............................................... 49

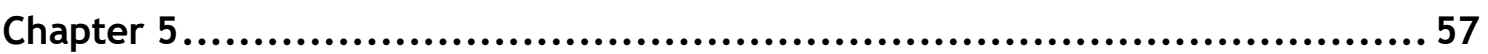

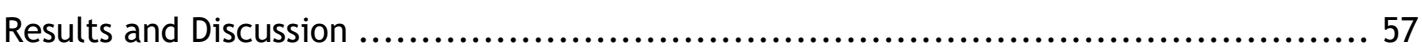

5.1 Pros and Cons of the Algorithm Steps ........................................ 57

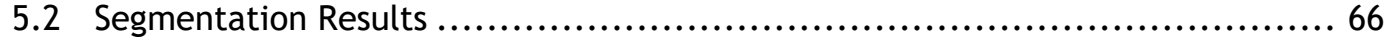

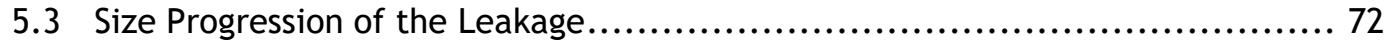

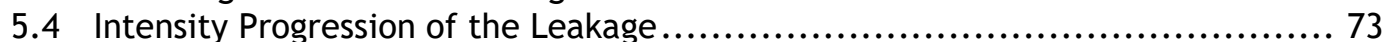

5.5 Computational Time of the Algorithm ....................................... 74

Chapter 6........................................................................ 77

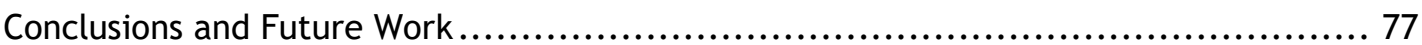

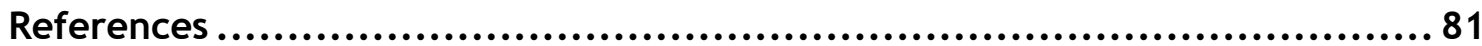

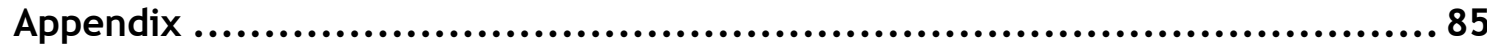

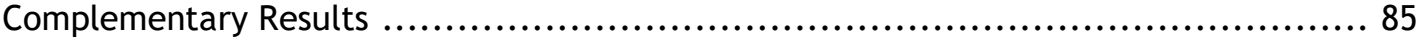




\section{List of Figures}

Figure 2.1 - Schematic cross-section of the human eye. ..................................

Figure 2.2 - Summary diagram of the major functions of RPE. ............................6

Figure 2.3 - Schematic representation of the central serous chorioretinopathy. ..............

Figure 2.4 - FA image with CSC leakage (orange circle). ................................

Figure 3.1 - Two different moments of two angiographic sequences where it is possible to visualize the differences between the two different types of leaks..................... 12

Figure 3.2 - Different appearances of FA images at different moments (0.39 min, 1.07 $\mathrm{min}, 3.52 \mathrm{~min}$ and $8.46 \mathrm{~min}$ ) of a CSC sequence. ................................... 13

Figure 3.3 - Steps of comparative methods by image subtraction. ......................... 14

Figure 3.4 - Steps of comparative methods by classification. ............................ 14

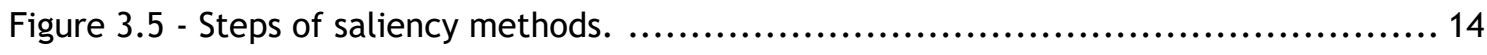

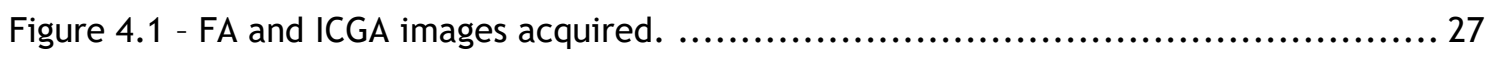

Figure 4.2 - Diagram representing the implemented process of the quantitative assessment of CSC leakages in sequences of FA images................................ 28

Figure 4.3 - Example of frame selection in an angiographic sequence ....................... 31

Figure 4.4 - Time recognition for less than (a) and greater than (b) 10 minutes. .............. 32

Figure 4.5 - The border of the image before and after the application of denoising

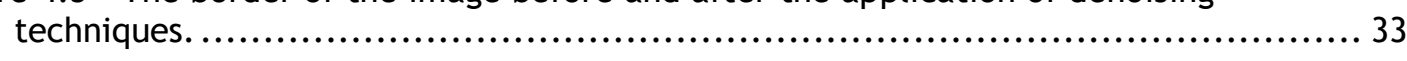

Figure 4.6 - Example of implementation of the registration developed. .................... 33

Figure 4.7 - Haar wavelet filters to compute the responses in $x$ (left) and y (right) [back regions and white regions are equal to -1 and 1 respectively].......................... 35

Figure 4.8 - Representative images of the registration process: target image (a), source image (b), registered image (c), mean of the registered image and the target images (d) and mosaicism between the registered image and the target image (e) .............. 36 
Figure 4.9 - Steps of the vessel segmentation algorithm: original image (a), preprocessing

(b), detection of the centerlines (c) and final segmentation of vessels (d)

Figure 4.10 - Steps of the candidate selection in the early frames: original image (a), original image with detected regions (b), binary detected regions (c), image after vessel segmentation algorithm (d) and final binary vessel map used in the vessel inpainting (e).

Figure 4.11 -Steps of vessel inpainting: input image (a), input image with vessels replaced by 0 (b) and image after vessel inpainting (c) . ..................................... 40

Figure 4.12 - Example of a window used in vessel inpainting.

Figure 4.13 - Regions $1 / 6$ of the width of the image border compared in the first step of the optic disc detection algorithm: image with the segmented vessels on the left border (a), first frame on the left border (b), image with the segmented vessels on the right border (c) and first frame on the right border (d).

Figure 4.14 - Diagram representing the sliding window (representing $i$ the window number and $\mathrm{m}$ an arbitrary value).

Figure 4.15 -Plot of the variation of the angle of inclination as the sliding window descends in the analysis region.

Figure 4.16 - Several steps of the algorithm with the top, the center and the bottom of the optic disc marked (red circle): first frame (a), region after step 1 (b), region after step 2 (c) and region after the last step (d).

Figure 4.17 - Example of a situation where the luminosity affects the intensity of a leakage: early image (a) and late image (b).

Figure 4.18 - Example of intensity normalization: before (a) and after (b). 47

Figure 4.19 - Several steps of the background removal: image with inpainted vessel (a), background estimation with morphological opening (b), background removal (c).

Figure 4.20 - Image subtraction steps: first image of the sequence (a), last image of the sequence (b), mean of the early frames after removal of background and optic disc (c), mean of the late frames after removal of background and optic disc (d) and subtraction between late and early processed frames (e).

Figure 4.21 - Candidates identification with Otsu method: last frame of the sequence (a), subtraction between late and early processed frames (b), rescaled (0 to 1$)$ image of the subtraction between late and early processed frames (c) and segmentation after Otsu method (d).

Figure 4.22 - Images of several steps until the region growing segmentation: late frame of the sequence (a), Segmentation of the lesion after the Otsu method (yellow) and segmentation of the lesion after the region growing method (yellow and red) (b), late frame of the sequence with the contour of the segmentation after the Otsu method (c) e after the region growing method (d).

Figure 4.23 - Effect of luminosity on apparent leakage size at different times: 9.4 minutes (a) and 9.6 minutes (b).

Figure 4.24 - Decision tree for the contraction bias.

Figure 4.25 - Contour progression of the lesions in the segmentation algorithm: last frame of the sequence without contour (a) and with contour (white - contour after Otsu 
method, yellow - contour after region growing, green - contour after active contour algorithm) (b); second-last image of the sequence without contour (c) and with contour (green - contour of the frame immediately later, white - contour after active contour algorithm).

Figure 5.1 - Intermediate and final results of the developed algorithm. ...................6 60

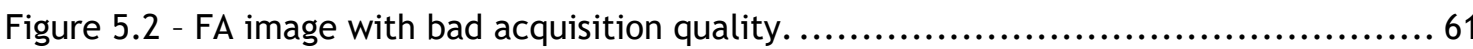

Figure 5.3 - Example of a registration that had minor distortions: target image (a), source image (b), registered image (c) and mean between the target and registered images (d).

Figure 5.4 - Example of a vessel segmentation that had some problems: original image (a and $b$ ), vessel tree with connected vessels and filling problems (c) and vessel tree with leakage detection and filling problems (d).

Figure 5.5 - Example of detecting a region that does not correspond to leakage (yellow circle).

Figure 5.6 - Example of a situation where there is a region that is blurred and the leakage intensities decrease a little: vessel inpainting image (a), output image (b).

Figure 5.7 - Algorithm segmentation results: original images $(\mathrm{a}-\mathrm{c})$, worst segmentation (b) (yellow - manual annotation, green - achieved segmentation), best segmentation (d) (yellow - manual annotation, green - achieved segmentation).

Figure 5.8 - Example of difficult determination of the region by presenting a haze region with large dimensions and different intensities: original images (a), original image with segmentation result (b) (yellow - manual annotation, black - achieved segmentation).

Figure 5.9 - Example of a sequence without a leakage: first frame (a), last frame (b). 70

Figure 5.10 - Example of a test image: original image (a), original image with annotations (b) (yellow - manual annotation, black - estimated segmentation of the leakage 1, white - estimated segmentation of the leakage 2 , red - possible missing leakage regions).

Figure 5.11 - Leakage with dark regions inside: original image (a), original image with the dark regions marked (b) and the leakage segmentation with the implemented method (red) and the manual annotation (yellow) (c).

Figure 5.12 - Size progression of different leakages in different sequences.

Figure A.1 - F sequence: original image with the segmentation achieved (in red) (left) and without (right); early frame (a), late frame (b).

Figure A.2 - S sequence: original image with the segmentation achieved (in red) (left) and without (right); early frame (a), mid-time frame (b) and late frame (c).

Figure A.3 - H sequence: original image with the segmentation achieved (in red) (left) and without (right); early frame (a), mid-time frame (b) and late frame (c).

Figure A.4 - B sequence: original image with the segmentation achieved (in green) and annotated manually (in yellow) (left), and without (right); early frame (a), mid-time frame (b) and late frame (c). 
Figure A.5 - C sequence: original image with the segmentation achieved (in purple) and annotated manually (in yellow) (left), and without (right); early frame (a), mid-time frame (b) and late frame (c)....

Figure A.6 -G sequence: original image with the segmentation achieved (with white in (a) and (b), and black in (c)) and annotated manually (in yellow) (left), and without (right); early frame (a), mid-time frame (b) and late frame (c).

Figure A.7 - A sequence: original image with the segmentation achieved (in red) (left) and without (right); early frame (a), mid-time frame (b) and late frame (c)................ 91

Figure A.8 - E sequence: original image with the segmentation achieved (in red) and annotated manually (in yellow) (left), and without (right); early frame (a), mid-time frame (b) and late frame (c).

Figure A.9 - D sequence: original image with the segmentation achieved (in red) and annotated manually (in yellow) (left), and without (right); early frame (a), mid-time frame (b) and late frame (c). 


\section{List of Tables}

Table 3.1 - Schematic/Summary representation of the state of the art for leakage

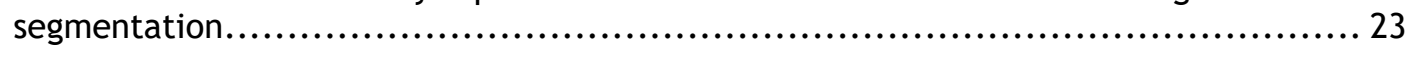

Table 4.1 - Acquisition times of different image series..................................... 26

Table 4.2 - Type of input images in each step of the implemented algorithm ................ 28

Table 4.3 - Conditions to guarantee monotony of leaks growth.............................. 56

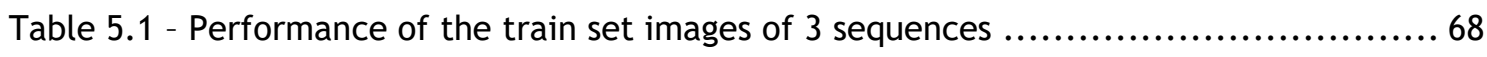

Table 5.2 - Performance of the test set images that have manual annotations................ 71

Table 5.3 - Computational time of the algorithm...................................... 75 


\section{Abbreviations}

$\begin{array}{ll}\text { ACC } & \text { Accuracy } \\ \text { ACWE } & \text { Active contour without edges } \\ \text { ART } & \text { Automatic real-time mode } \\ \text { AUC } & \text { Area under the curve } \\ \text { CAD } & \text { Computer-aided diagnosis } \\ \text { CHT } & \text { Circular Hough transform } \\ \text { CI } & \text { Convergence index } \\ \text { CNV } & \text { Choroidal neovascularization } \\ \text { CSC } & \text { Central serous chorioretinopathy } \\ \text { CV } & \text { Coefficient of variation } \\ \text { DME } & \text { Diabetic macular edema } \\ \text { DSE } & \text { Dice similarity coefficient } \\ \text { ED-DB-ICP } & \text { Edge-driven dual-bootstrap iterative closest point } \\ \text { FA } & \text { Fluorescein angiography } \\ \text { FEUP } & \text { Faculty of Engineering of the University of Porto } \\ \text { FN } & \text { False negative } \\ \text { FoV } & \text { Field of view } \\ \text { FP } & \text { False positive } \\ \text { GMM } & \text { Gaussian mixture model } \\ \text { ICGA } & \text { Indocyanine green angiography } \\ \text { IPACHR } & \text { Infinite perimeter active contour with hybrid region } \\ \text { JI } & \text { Jaccard index } \\ \text { LP } & \text { Laser photocoagulation } \\ \text { MI } & \text { Mutual information } \\ \text { MR } & \text { Macular retinopathy } \\ \text { MSAC } & \text { M-estimator sample and consensus } \\ \text { NCC } & \text { Normalized cross correlation } \\ \text { NMI } & \text { Normalized mutual information } \\ \text { OCT } & \text { Optical coherence tomography } \\ & \end{array}$


OD

OLS

PDT

$\mathrm{RF}$

ROC

RPE

SBF

SIFT

SLIC

SN

SSD

SSE

SP

SURF

$\mathrm{TaP}$

TN

TP
Optic Disc

Ordinary least squares

Photodynamic therapy

Random forest

Receiver operating characteristic

Retinal pigment epithelium

Sliding band filter

Scale invariant feature transform

Simple linear clustering

Sensitivity

Sum of squared differences

Sum of squared errors

Specificity

Speeded up robust features

Target pixel

True negative

True positive 


\section{Chapter 1}

\section{Introduction}

\section{$1.1 \quad$ Background}

Vision is one of the most complex mechanisms of the human body. The eyes capture a huge amount of information and a large part of the brain is needed to process it and put it into use. The interpretation of information provides the characteristics of the visual stimulus as shape, color, size, texture, weight, and a meaning. The diseases or problems that appear in the eyes should be prevented, diagnosed, and treated because visual perception can be compromised and cause problems in daily life.

The most problematic eye diseases come from problems in the retina. It is a thin layer of tissue, located in the inner part of the eye. Any type of change may indicate risk of partial or total loss of vision because the retina is the part of the eye where the photoreceptors are, i.e., the cells that have as function to transmit to the brain the luminous stimuli to be transformed into visual stimuli [1].

The central serous chorioretinopathy (CSC) is an important retinal disease characterized by subretinal accumulation of choroid fluid through a retinal pigment epithelium (RPE) lesion [2]. A detachment of the neural retina (photoreceptors and other cells associated with the sensing and processing of light stimulus) allows this leakage [3, 4]. In this case, the diagnosis and the adequate follow-up of the CSC are also important to know the size, the evolution, and the severity of the leak and to decide the treatment.

Fluorescein angiography (FA) is the most widely used imaging modality for the diagnosis and the visualization of leaks in retinal diseases. A fluorescent dye is injected into the bloodstream and highlights the normal and the abnormal structures of the ocular fundus that interact with fluorescein so they can be photographed. If the retinal pigment epithelium or retinal vascular system have problems, leakages can be identified in FA images [5]. In the past years, computer- 
aided diagnosis (CAD) came as a strong complementary tool to support the diagnosis in retinal diseases and the leaks became easily, quickly, and automatically detected and quantified.

\subsection{Motivation}

The central serous chorioretinopathy is one of ten most common diseases of the posterior segment of the eye and a frequent cause of mild to moderate visual acuity. Although cases of acute CSC usually can be solved in a few months and without severe visual problems, recurrent or chronic CSC can lead to permanent loss of visual acuity [2]. Indeed, vision acuity is affected not only by the duration of the disorder but also by the height of the detachment [6] because larger detachments lead to larger leaks and a greater damage to the photoreceptors. Therefore, detection and evaluation of these retinal abnormalities are essential for a proper follow-up and treatment of the disease.

In the CSC, fluorescein angiography shows rapid filling of the subretinal neovascular net [7] and a leakage point in the early phases. The dye leakage area in images increases along the exam because the fluorescein passes through small breaks in the RPE until it slowly reaches the subretinal space and interacts with the leakage of fluid. Thus, fully leakage is only visible in the late phases of the exam. This area of leakage measured in FA images is correlated to the height of the neurosensory detachment [8]. Therefore, fluorescein angiography is a powerful imaging modality to identify the presence, location, and size of the CSC lesions. The analysis and the interpretation of FA sequences have been performed by ophthalmologists or other health graduate technicians. Usually, the ophthalmologist selects only three frames from the acquired images (early, mid, and late phases) and qualitatively observes the dye leakage. Important temporal information can be lost. Furthermore, manual segmentation is often used and it is a tedious, subjective, and time-consuming process. Lack of reproducibility in intra and inter subject is also a problem due to the dependence on the ophthalmologist own expertise [9].

For these reasons, there is a strong need to provide the ophthalmologist with an automatic CAD tool for a better analysis of CSC leaks in FA images. There is a hope in a substantial improvement in health care with this CAD tool since the prevalence and effects of CSC are currently quite considerable.

\subsection{Objectives}

This work uses fluorescein angiography sequences to find central serous chorioretinopathy leaks. If there is a CSC lesion, the dye leakage area increases throughout the exam. Then, one of the objectives of the work is to develop a CAD algorithm with the ability to detect the presence of lesions and, if present, to segment and calculate the area of the leaks in all images 
of the angiographic sequence. Another objective of the work is to analyze the growth of lesions through the examination.

\section{$1.4 \quad$ Structure of the Report}

This work has five other chapters besides this first, the introduction, where it is presented the context, motivation, and objectives of the work.

As the master thesis is focused on the automatic detection of CSC leaks in FA images, in chapter 2 , the anatomy of the eye is described since it is essential to understand the processes and terms associated with the disease. The etiology, the consequences, the prevalence, the symptoms, the risk factors, the treatments, and the diagnostic options of the disease are also presented.

Chapter 3 presents the challenges inherent to the characterization of the disease as well as a literature review of the main methods already developed for the characterization of leaks.

In chapter 4 , the data set is described. Likewise, in this chapter, the methodology implemented to reach the segmentation and characterization of leaks is presented. The methodology has two mains parts: steps that allow the detection of leakage candidates and the leakage segmentation itself. Each step is explained in detail.

Results are shown in the chapter 5 . There, the achieved segmentation of the leaks is compared with the manual annotations performed by an ophthalmologist and graphs of size and intensity progression of the lesion over time are presented. The results are also analyzed, and the pros and cons of each step of the algorithm are identified.

The last chapter comprehends conclusions notes about the results and the accomplished work. In addition, some suggestions for future work are made. 


\section{Chapter 2}

\section{Central Serous Chorioretinopathy}

\subsection{Eye Anatomy}

Eyes allow humans to have the perception of the outside world. Three layers of tissue enclose the eye (figure 2.1):

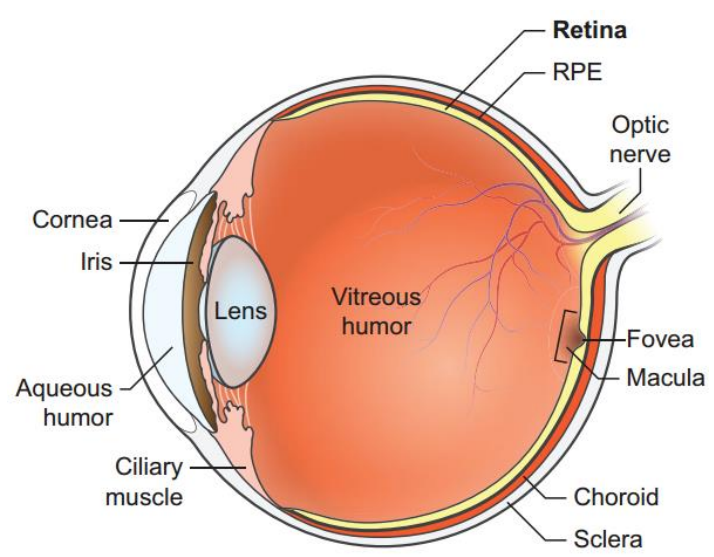

Figure 2.1 - Schematic cross-section of the human eye. Image source: [10].

- Sclera: This is the exterior layer of the eye and a tough white fibrous tissue. It provides structure, protection, and strength to the eye. The cornea, an anterior portion of sclera, is transparent and permits the entrance of light rays into the ocular globe;

- Choroid: It is highly vascularized and continuous with the iris (the colored part of the eye within central opening - pupil). The main objective of this layer is enabling the diffusion of nutrients and oxygen to the retina and regulate ocular pressure and temperature [11]. The structure of the choroid is generally divided into four layers: Haller's layer (large diameter vessels), Sattler's layer (medium diameter vessels), choriocapillaris (small capillaries) and 
Bruch's membrane, from the outer to inner layers [12]. Bruch's membrane plays an important role in the visual acuity because it acts a blood-retinal barrier;

- Retina: This is the layer where the processing of light signals is performed, due to the presence of photoreceptors (type of neurons capable of phototransduction). There are two types of photoreceptors in the human retina: rods (responsible for vision at low light levels) and cones (active at higher light levels). The retinal pigment epithelium, the outermost layer of the retina, provides nutrition (glucose and essential ions) and protection (contains melanin which protects from damaging levels of light) to photoreceptors (figure 2.2). In the center of the retina is the optic disc (OD) (blind spot without photoreceptors) which is the beginning of the optic nerve (which conducts the transduction signal to the brain) and the entry point for the major blood vessels that supply the retina. Near the lateral edge of the optic disc lies a circular portion called macula, that is essential to central vision (responsible for the visualization details). At the center of the macula lies a depression called the fovea [1]. The fovea includes also the foveola which is the region of the eye with highest visual acuity because it contains the highest cones density; rods and blood vessels are absent.

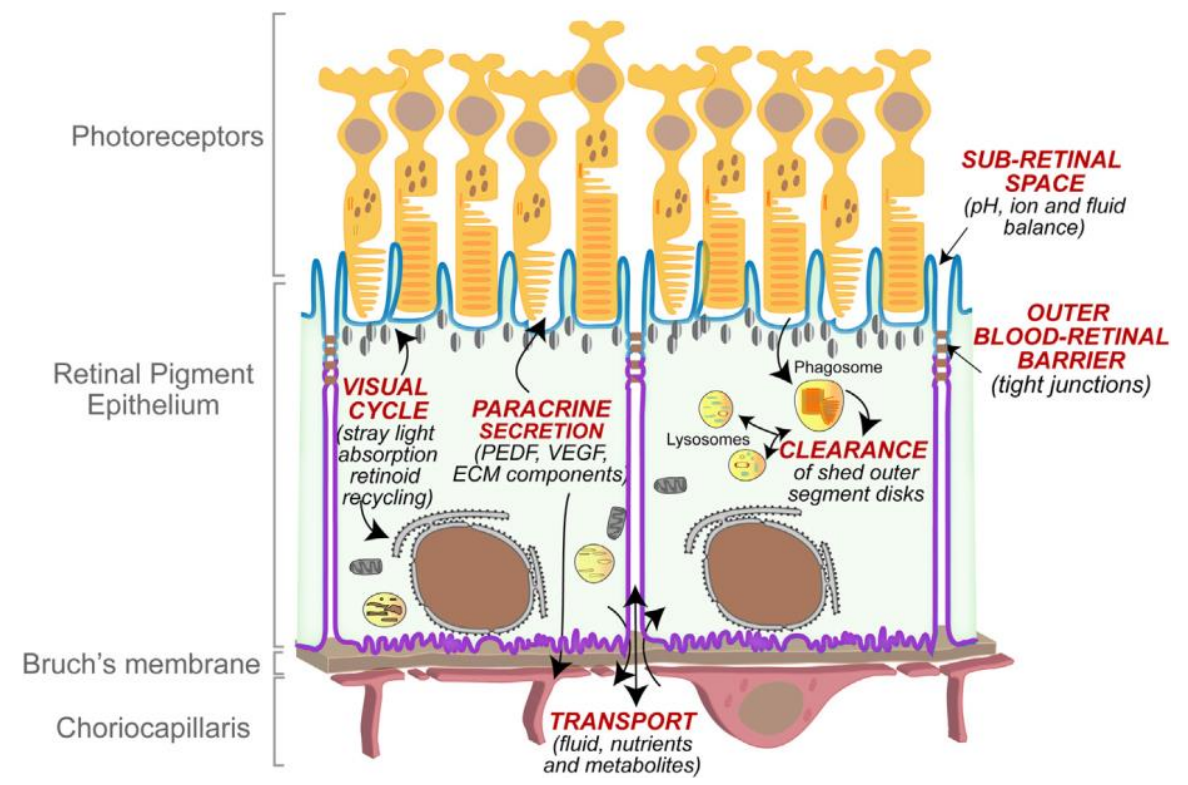

Figure 2.2 - Summary diagram of the major functions of RPE. Image source: [13].

Indeed, the eye is a complex structure. The nutrition of intraocular tissues, particularly the retina, is assured by the retinal arteries and the choroid blood vessels. The choroid receives the greatest blood flow (65\%-85\%) and the remaining 15\%-35\% flows to the retina through the central retinal artery to nourish the inner retinal layers [14]. The retinal pigment epithelium and the Bruch's membrane are essential in this process. They mediate the transport of nutrients to the outer retina (particularly the photoreceptors) and from waste to the choroid. As there is a space between the RPE layer and the rest of the retina, this anatomic arrangement is mechanically unstable. If defects arise in the RPE, nutrients and fluids can be unproperly 
distributed and the photoreceptors can be damaged because they may not receive the usual nutrition. The integrity of photoreceptors is essential to preservation of visual acuity.

\subsection{Central Serous Chorioretinopathy Description}

Central serous chorioretinopathy is an ocular disease in which there is a serous detachment between the neurosensory retina and the retinal pigment epithelium (figure 2.3). The detachment comes from the accumulation of fluid in the subretinal space due to defects in the RPE [15]. It can be characterized by the presence of mono (one) or paucifocal (some) RPE lesions [5] and is usually manifested in one eye.

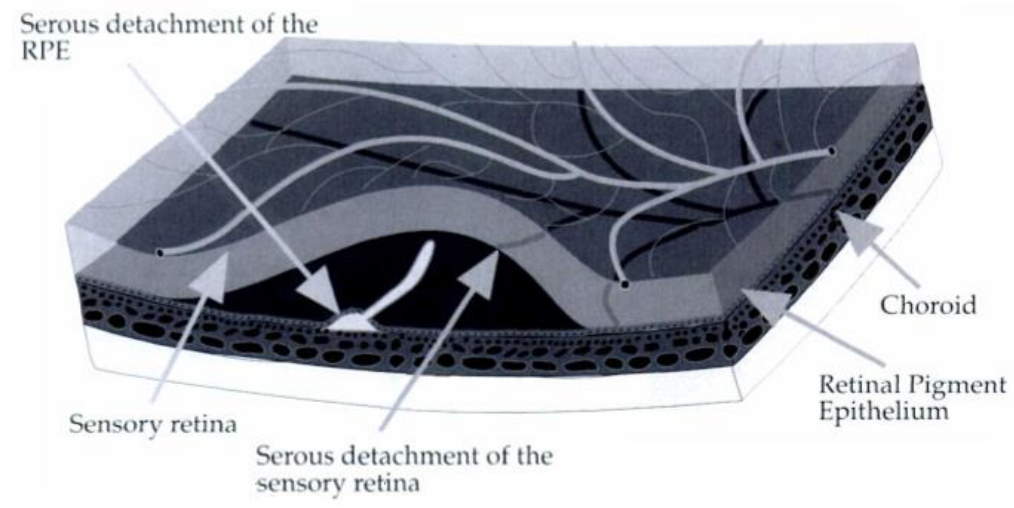

Figure 2.3 - Schematic representation of the central serous chorioretinopathy. Image source:

[7].

Patients with CSC are typically male (male to female ratio is approximately 6:1) [16] with mild-life age (20-50). However, precise data on its prevalence are not available [17].

The leakage of fluid through the RPE often occurs at the macula, resulting in central vision loss (vision becomes without details), disturbed color vision, intolerance to bright sunlight, central scotoma, metamorphosia and/or micropsia [16]. A spot-like dark area in the centre of the field of vision appears in central scotoma, straight lines appear wavy in metamorphosia and objects are perceived to be smaller than they are in micropsia [18].

Two main forms of CSC can be distinguished: acute and chronic. Acute CSC typically disappears naturally within a few months with total reabsorption of the subretinal fluid, visual acuity is recovered close to premorbid values but a small impairment in contrast sensitivity and color discrimination may be maintained. A single episode of acute CSC may be converted to multiple recurrences of the serous retinal detachment. Eyes with chronic CSC persist for more than three months. The persistent presence of the subretinal fluid associated with a progressive deterioration of the RPE functions may be a cause of vision loss secondary to progressive retinal damage [17]. 
The exact etiology is unknown but conditions like use of corticosteroids, the stress and hypertension are identified as main risk factors for CSC [16]. In general, laser photocoagulation (LP) and photodynamic therapy (PDT) are used to reduce the time recovery and the recurrence, when disease does not improve spontaneously during observation. Laser photocoagulation uses the heat from a laser to seal or destroy abnormal, leaking blood vessels in the retina. In PDT, a drug, injected into the patient's arm, reacts to light. Once it moves up to the eye, it pools in damaged blood vessels of the retina. When exposed to the laser, the drug seals off the leaking vessels.

\subsection{Imaging Modalities for CSC Diagnosis}

Fluorescein angiography (FA) is the most common image modality used for the detection and follow-up the CSC. However, indocyanine green angiography (ICGA) and optical coherence tomography (OCT) are also used.

In FA, there is a camera equipped with excitation and barrier filters. Fluorescein dye is injected intravenously. White light from a flash is passed through a blue excitation filter. Blue light (wavelength $465-490 \mathrm{~nm}$ ) is then absorbed by unbound fluorescein molecules and molecules fluoresce, emitting light with a longer wavelength in the yellow-green spectrum (520-530 nm) [19]. The walls of retinal blood vessels are transparent and, in the CSC leaks, dye molecules can pass through the choriocapillaris and penetrate through the defects of the RPE. CSC leaks exhibit a hyperfluorescence behavior (abnormal presence of fluorescein at a given time and place). So, in FA grayscale images, vessels and leaks appear bright and the other structures of the retina appear dark (figure 2.4). The complete filling of the vessels is accomplished until 1 minute after the injection of the dye and it remains until approximately 10 minutes. The CSC leak is called pooling because fluorescein gradually fills a fluid-filled space. The dye leakage increases throughout the examination and it is not possible to visualize it completely in the first moments of analysis of the FA images (unlike the vessels) because the dye molecules travel from the choriocapillaris (choroid), they pass through small defects of $\mathrm{RPE}$, until the retinal space is reached. The diameter of the regions corresponding to the leakage in FA images often varies between $100 \mu \mathrm{m}$ and $1000 \mu \mathrm{m}$. Various patterns of dye leakage are seen like the smoke-stack and the ink-blot. On one hand, a smoke-stack leakage increases only vertically as the fluorescein angiography exam progresses. On the other hand, an ink-blot leakage increases both vertically and horizontally in the fluorescein angiographic sequence [20]. Despite the general acceptance of FA, its application must be restricted because of the possibility of severe complication and the discomfort to the patients, as well as the time needed to make the exam [21]. 


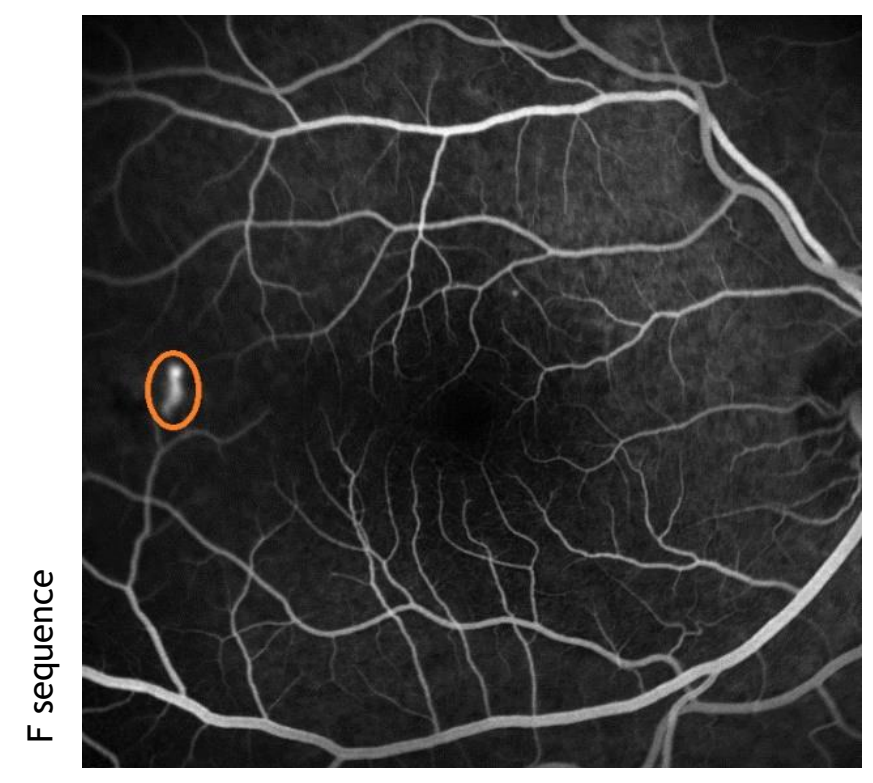

Figure 2.4 - FA image with CSC leakage (orange circle).

In ICGA, a tricarbocyanine dye is injected into the bloodstream but indocyanine green fluoresces with the infra-red light. The infra-red wavelengths can penetrate the retinal layers making the circulation in deeper layers visible when photographed with an infra-red sensitive camera [22]. So, ICGA allows the visualization of changes involving the deeper choroidal vascularization. Although it does not contribute to treatment deliberations, it is particularly important in chronic CSC patients, because sometimes it is very difficult to interpret areas of leakage on FA images, and in patients with no marks of fluorescein, because the increased choroid vascular hyperpermeability can be a sign of the disease [3]. Indeed, numerous recent studies using ICGA allow to describe abnormalities of the choroidal circulation [22].

OCT is an image diagnosis modality which allows ophthalmologist to take a cross-section image of tissues in the back of the eye. It uses a reflected light from a low power laser [23]. OCT is a noninvasive image modality and it is usually performed to visualize the morphologic changes involving the retinal layers [17]. In contrast to FA and ICGA imaging, OCT angiography catches movements of blood cells, instead of serum detachments or staining of vessel walls and tissue. As OCT can provide more information about the blood supply in CSC, it can be useful to better understand the underlying etiology of the disease [16]. 


\section{Chapter 3}

\section{State of the Art}

\subsection{Challenges}

In the FA images, the lesions usually appear bright as well as the blood vessels and the remaining structures appear dark. However, several phenomena may occur in the image acquisition during the sequence, as described below, and they change the intensity of anatomical structures, making the characterization of lesions as a very challenging problem.

There are two distinguishable leakage types in FA images. Figure 3.1 shows the two types. The times between the two images of each sequence are similar to allow comparison of the two types of dye leaks. One, which is the most common, increases considerably in size and intensity and is only identifiable at later stages of the examination ( $\simeq 3 \mathrm{~min}$ ) (figures 3.1 (a) and (b)), and the other is visibly identifiable very early ( $\simeq 1 \mathrm{~min})$ and does not increase much in size throughout the examination (figures 3.1 (c) and (d)).

The complete filling of the blood vessels with fluorescein does not occur until approximately one minute after injection (early phase). Around ten minutes, it is often difficult to distinguish between the background and the vessels due to loss of fluorescence (late phase). The mentioned times are only approximate, so there is a slight delay or an advance in the fill, or in the final loss of fluorescence. As the anatomical structures present in FA images are only differentiable between these two moments (mid phase), the analysis and detection of the leaks must also be confined between these times. Figure 3.2 shows four images of the same sequence from a fluorescein angiography exam. Figure 3.2 (a) represents a situation where vessels are not yet fully filled and it should not be used in the leakage segmentation process. Figure 3.2 (b) is an image of the first minutes of the exam where it is still not possible to visibly detect the presence of lesions but that can be useful if techniques of comparison between frames are used for achieving the segmentation of the leak. In figure 3.2 (c), leak is visibly identifiable. 

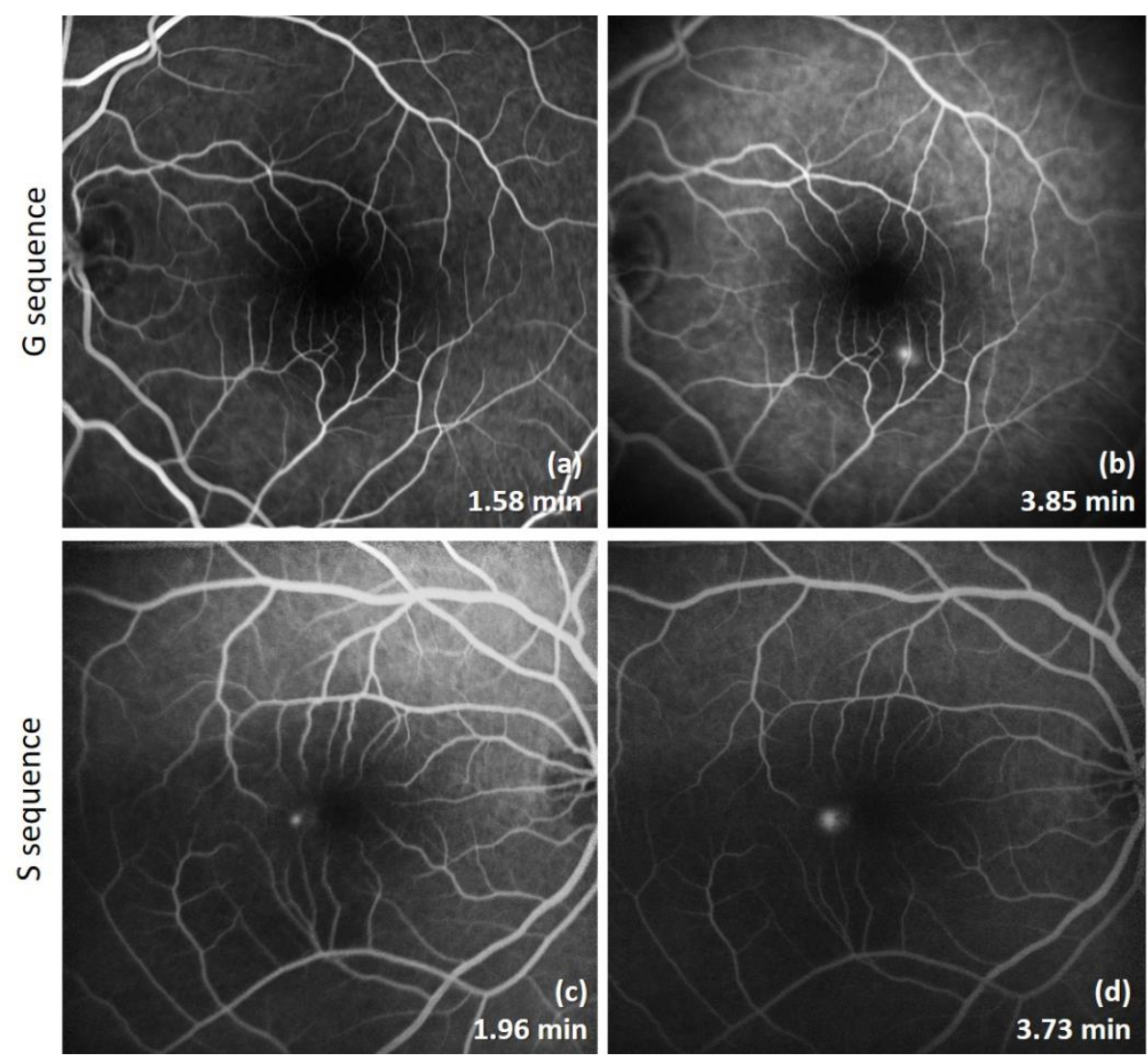

Figure 3.1 - Two different moments of two angiographic sequences where it is possible to visualize the differences between the two different types of leaks.

One of the challenges of the automatic characterization of leaks is to distinguish them from the blood vessels because they have both the similar pixel intensities and leaks can even overlap the blood vessels. However, dye leakages increase in size throughout the examination (unlike the vessels) and sometimes they may not be really visible in the early stages. The influence of the optic disc in the detection of lesions is, in turn, a more complicated challenge to overcome because late staining of the optic disc is a normal finding. Therefore, the intensity and the size of the border of the optic disc seem to increase. This is a similar behavior to one of the lesions. In figure 3.2 (d), the leak overlaps the vessels. Moreover, the optic disc has late staining (inside the drawn red shape).

Additional challenges arise due to the movement of the eye during the exam. This complicates the use of the comparison techniques because the anatomic structures are not at the same position in all frames and images may also become blurred.

At last, the intensity of the anatomical structures may differ along the angiographic sequence due to the natural variations of the fluorescence over time, phenomena that may occur in the choroidal layer and changes in the conditions of the imaging acquisition system. Indeed, the incident angle of the laser beam may be different at different time points. In addition, with the ocular movements, already referenced, the intensities may vary even more because the luminosity conditions are then modified. The areas of great differences should be 
just the lesions, but as shown in figure 3.2 (d), the intensity of the background greatly changed compared to figure 3.2 (b).
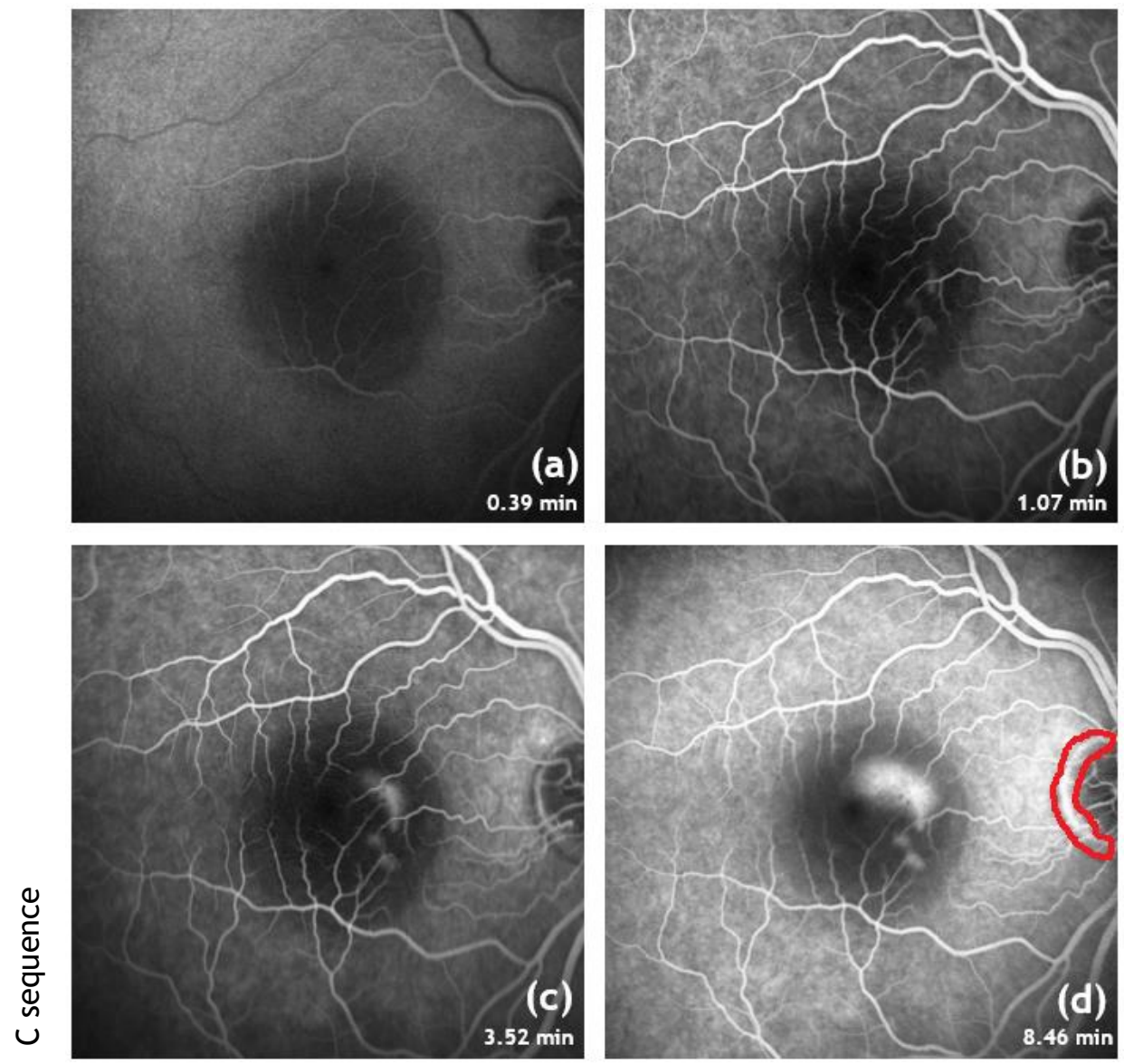

Figure 3.2 - Different appearances of FA images at different moments $(0.39 \mathrm{~min}, 1.07 \mathrm{~min}$, $3.52 \mathrm{~min}$ and $8.46 \mathrm{~min}$ ) of a CSC sequence.

\subsection{Leakage Assessment: a Literature Review}

In the review of the literature no study was found relating to an automatic CAD tool to characterize dye leaks of CSC in FA images. However, the leakage segmentation for diabetic macular edema (DME), choroidal neovascularization (CNV) and macular retinopathy (MR) is similar to the problem of CSC leakage segmentation. In DME, there is an abnormal permeability of the blood-retina barrier which results in leakage and accumulation of fluids in the retina layer. In CNV, new (abnormal) blood vessels start to grow in the choroidal layer beneath the center of the macula and, as they grow, they leak fluid or blood. MR is any pathological condition of the macula. Although MR can reveal other types of angiographic vascular abnormalities, the situations similar to CSC, which are described in this chapter, are large focal lesions (circular areas where the greatest linear diameter is larger than $125 \mu \mathrm{m}$ ) with late 
hyperfluorescence. Even for these diseases, only a few algorithms were developed for the detection of leaks.

Leakage characterization algorithms can be divided into two main categories: comparative methods and saliency methods [24, 25]. Comparative methods can be further divided into image subtraction approaches $[9,26]$ or classification approaches [27-28]. Comparative methods use temporal information because the variation of the size and the intensity over time in the sequence happens mainly in the lesions. Saliency methods are based on the perceptual qualities that make an element stand out relative to its neighbors, often associated to variations in image attributes like color, gradient, edges, and boundaries. In this case, the leaking regions may be defined as high salience regions because the leakage of fluorescent dye causes a considerable difference in brightness between the leak and surrounding non-leaking areas. The steps of the comparative methods by image subtraction, the comparative methods by classification and the saliency methods are presented in figures 3.3, 3.4 and 3.5 respectively. Table 3.1 (at the end of the chapter) summarizes state of the art methods that can be used for the segmentation of leaks in FA images.

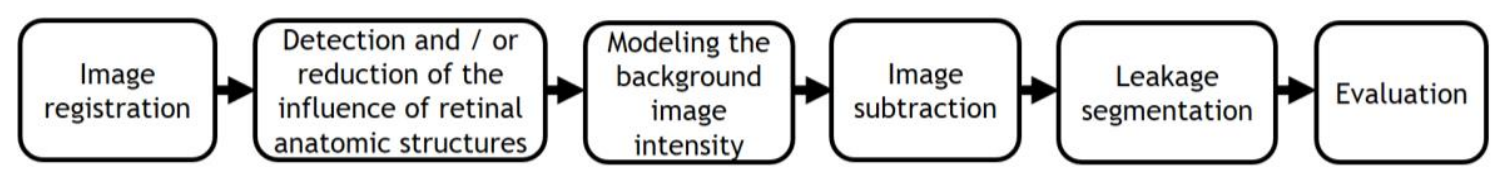

Figure 3.3 - Steps of comparative methods by image subtraction.

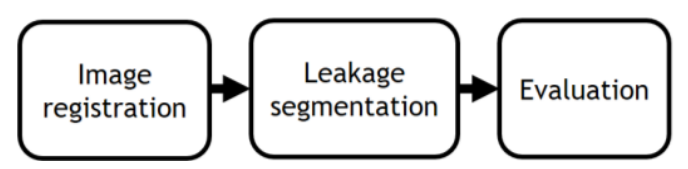

Figure 3.4 - Steps of comparative methods by classification.

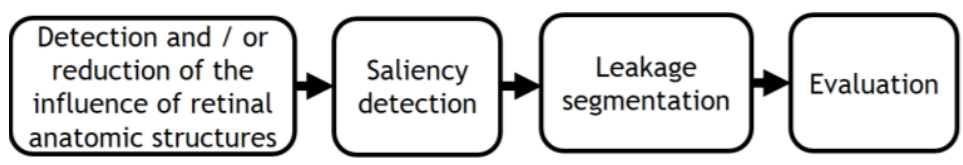

Figure 3.5 - Steps of saliency methods.

As comparative methods use temporal information, registration is essential to have the same retinal structures in the same position in all images. Registration is only omitted from studies if images appear to be well aligned after the acquisition. On other hand, in the saliency methods, this step is irrelevant since the location of the lesion is determined individually in each image.

The methods with classification compare the intensities of the pixels at same position along the angiographic sequence. Then, the pixel intensity values in the images at specific times will be the mainly extracted features. Classification methods use supervised learning or 
unsupervised learning. Supervised learning uses a priori knowledge involving the ground truth (GT) of a training set of images. In unsupervised learning, there is no idea what results should look like. With training, supervised classifiers may overcome the challenges of the similar pixel intensities of the leaks and the vessels, the late staining of the optic disc and the background intensity variation. On the other hand, in the subtraction methods and saliency methods, the anatomical elements need to be detected and their influence must be reduced in the final segmentation to overcome the challenges of the characterization. Likewise, the variation of the background intensity should be reduced in the subtraction approaches. Finally, segmentation and evaluation are steps common to all approaches.

\subsubsection{Frame Selection}

Manual choice, time-based choice and intensity-based choice have been the main strategies used for frame selection. The first approach allows an effective selection of the images but is a non-automatic method that spends attention and time of the healthcare professional. The second approach selects automatically the middle phase images of the exam (from 1 to 10 minutes). The last approach uses measures of intensity similarity between images. The measures of similarity can include sum of squared differences (SSD) (equation 3.1), normalized correlation coefficient (NCC) (equation 3.2), mutual information (MI) (equation 3.3) and normalized mutual information (NMI) (equation 3.4) between two images.

$$
S S D=\sum_{i} \sum_{j}[f(i, j)-g(i, j)]^{2}
$$

where $f(i, j), g(i, j)$ are $M \times N(i=1,2, \ldots, M ; j=1,2, \ldots, N)$ images.

$$
N C C=\frac{\sum_{i} \sum_{j} f_{c}(i, j) g_{c}(i, j)}{\sqrt{\sum_{i} \sum_{j}\left(f_{c}(i, j)\right)^{2} \sum_{i} \sum_{j}\left(g_{c}(i, j)\right)^{2}}}
$$

where $f_{c}(i, j)=f(i, j)-\bar{f}, g_{c}(i, j)=g(i, j)-\bar{g}$ and, $\bar{f}$ and $\bar{g}$ are the mean value of image $f$ and $\bar{g}$ respectively.

$$
\begin{gathered}
M I=I(X, Y)=H(X)+H(Y)-H(X, Y)= \\
=-\sum_{k} P\left(x_{k}\right) \log _{2}\left(P\left(x_{k}\right)\right)-\sum_{z} P\left(y_{z}\right) \log _{2}\left(P\left(y_{z}\right)\right)+\sum_{k} \sum_{z} P\left(x_{k}, y_{z}\right) \log _{2}\left(P\left(x_{k}, y_{z}\right)\right) \\
N M I=\frac{I(X, Y)}{\sqrt{H(X) \cdot H(Y)}}
\end{gathered}
$$

where $P\left(x_{k}\right), P\left(y_{z}\right)$ represents the normalized histogram of two images; $P\left(x_{k}, y_{z}\right)$ represents the joint normalized histogram of the two images; $k, z$ are the number of levels in the histogram; $H(X), H(Y)$ are entropies of the images $\mathrm{X}$ and $\mathrm{Y}$ respectively and $H(X, Y)$ is joint entropy. 
H. Rabbani et al. [9] do the frame selection by removing frames with a normalized cross correlation less than 0.7 with the last frame.

The datasets used in the different algorithms, described in this chapter, contain only adequate images to achieve the segmentation. So, frame selection was not necessary.

\subsubsection{Image Registration}

State of the art algorithms for registration vary essentially in the type of similarity measures used and the type of transformation model to enable a source image to be changed to match the target. Generically, registration can be divided into feature approaches and intensity approaches. Intensity approaches match intensity patterns in each image using mathematical or statistical criteria. Features approaches frequently use identifiable anatomical elements or regular elements in each image. In fact, features approaches have been more widely used in retinal imaging. Usually they search for confidence points of the total image content or of the blood vessels to match in both frames (target and source). In the first, different match must not be concentrated in the lesion. In the second, it is necessary to detect the vessels before the registration.

Rigid, affine, and quadratic have been the major transformation models. Although these are used, movements in the retina can be approximated by a projective transformation. The retina surface can be considered almost spherical due to the shape of the eyeball and it only has slight depressions in the region of the macula and the optic disc. It is also reasonable to assume that the retina is rigidly attached to the back of the eye so the projective transformation can be supposed because all points in the surface move almost uniformly. Furthermore, the projective group englobes affine cases and rigid cases. Then, the projective transformation produces identical or better results than the previous two mentioned transformations.

In H. Rabbani et al. [9], speeded-up robust features (SURF) methodology [29] was performed in binary vessel maps previously constructed to detect scale and rotation-invariant interest points. After, these points are matched between the source and target images. Since SURF point detection is based on the Hessian matrix, interest points correspond to blobs, i.e., they mainly are in bifurcations of vessels. Outliers were excluded using M-estimator sample and consensus (MSAC) algorithm [30] and with remaining points an affine geometric transformation was found to apply to the source image. To improve the gross global registration, patch-based local registration was utilized too. The local registration was a multi-resolution approach applied on local $40 \times 40$ pixel rectangular patches.

M. S. El-Shahawy et al. [26] and A. S. Fahmy et al. [27] estimated the relative translation and rotation between the images using (rigid) phase correlation method. Phase correlation is a Fourier-based technique. Different rotations are applied to the source image to find the rotation parameter in which the source and the target images are just shifted. Two shifted 
images have the same Fourier amplitude and a phase difference proportional to the translation. However, the first also used an extracted vessel tree to align the frames and the second used consecutive original images.

C. Tsai et al. [28] adopted the Edge-Driven Dual-Bootstrap Iterative Closest Point (ED-DBICP) with a quadratic transformation [31] to map the total content of both images (target and source). As ED-DB-ICP uses the Lowe's scale invariant feature transform (SIFT) points from the gradient magnitude image and enriches the point descriptor with global-shape context, the non-linear intensity changes in the feature extraction and matching between images can be overcome. SIFT points are scale and rotation invariant too. As the SIFT points are detected based on difference of Gaussians, blob points on the vessel bifurcations are predominantly extracted.

\subsubsection{Vessel Segmentation}

Leaks must not be mistakenly considered and segmented together with the blood vessels especially those that overlap vessels. Different authors have applied very different vessel segmentation methods in FA images with leaks [9, 24-26].

H. Rabbani et al. [9] resorted to Dijkstra forest algorithm of Estrada et al. [32] to segment vessels. In their method, after preprocessing, in each iteration, the best unvisited vessel pixel in the image is repeatedly chosen as a starting point for a Dijkstra exploration of the unvisited part of the image, which results in a new tree in the growing forest of vessels. Forest growth stops when the best unvisited vessel pixel is worse than a predefined threshold.

In M. S. El-Shahawy et al. [26], vessels were enhanced with matched filters. After that, a fuzzy K-means classifier was used with number of clusters $(K=3)$ to extract vessels. The cluster from the original image with the highest intensity was consider containing the vessels.

Y. Zhao et al. [24], firstly, used local phase enhancement. Local phase enhancement filters are invariant to intensity differences within the image and produce more reliable results when compared to intensity based filters. To extract the vesselness map was used the graph cut method by Chan-Vese's model [33]. The Chan-Vese model is an active contour without edges (ACWE) region based segmentation method. The model begins with an initial contour in the image and then it evolves according to some evolution equation. The goal is to evolve the contour in such a way that it stops on the boundaries between the vessels and the background. Exterior pixels were removed iteratively by using a thinning algorithm and a least-squared cubic spline technique was used to obtain smoother trajectories for the centerlines in the final segmented vessels.

In Y. Zhao et al. [25], the infinite perimeter active contour with hybrid region (IPACHR) method [34] was used for segmenting retinal vessels. This method is also based on active contour, but considers the geometric information and the intensity of the image. The regularization of the infinite perimeter also helps to reach the retinal vasculature. 
Once the anatomical structures are detected, their influence in the final segmentation can be mitigated. H. Rabbani et al. [9] and M. S. El-Shahawy et al. [26] substituted the pixel values of blood vessels with surrounding background. In Y. Zhao et al. [24] and in Y. Zhao et al. [25], vessels are set to 0 (black) in the final segmentation process.

There are other approaches for segmenting blood vessels that have very good results but have not yet been tested in FA images with these types of leaks [35-37].

Mendonça et al. [35] proposed an approach based on centerline detection by using directional information from a set of four difference of offset Gaussian filters. After that, a region growing procedure guided by a few image statistics connects the candidate points. The final segmentation is achieved using an iterative region growing that mixes the contents of different binary images resulting from vessel width dependent morphologic filters.

Wang et al. [36] present a hybrid approach to obtain a vesselness map. A convolutional neural network was used for extracting a set of hierarchical features and, after that, ensemble random forests (RF) was trained to obtain a vessel classifier.

Qin Li et al. [37] present a multi-scale approach using Gabor filters and scales multiplication (the product of two Gabor filter responses at two adjacent scales). After that, a line tracking algorithm is used to guide the selection of the threshold to apply.

\subsubsection{Optic Disc Segmentation}

The optic disc is an entrance point for the major blood vessels that supply the retina. In FA images, it corresponds to a region with great number of crossing of blood vessels. So, the optic disc is often detected using segmentation information of vessel segmentation.

In Y. Zhao et al. [24] and in Y. Zhao et al. [25], the leakage segmentation is only performed on the last image of the sequence where the complete filling of the detachment has already occurred, so at this stage the optic disc should already present late staining. It thus appears as a bright region and should appear along with the vessel segmentation. Therefore, it was assumed to be a region where more than 5 vessels are crossed in these works.

There are algorithms for segmenting the optic disc that have not yet been tested in FA images with these types of leaks and these methods can provide a more accurate detection than the previously consideration [38-39].

Aquino et al. [38] proposed a template based method which matches the OD template with different parts of the input image and finds the similarity. This allow to define a sub-image and the OD boundaries were approximated using the Circular Hough Transform (CHT).

Cheng et al. [39] proposed a method which classifies each superpixel of the image as disc or non-disc. Superpixels are connected regions of the images that have similar intensity properties. Simple linear clustering (SLIC) was used to aggregate nearby pixels into superpixels. After that, the final contour is obtained with a deformable model. 


\subsubsection{Modeling the Background Intensity}

In H. Rabbani et al. [9], local histogram equalization and a top-hat filter (disk-shaped structuring element with a radius of 20 pixels) was applied to perform background normalization. The top-hat filtering computes the morphological opening of the image and then subtracts the result from the original image. Opening is an erosion (equation 3.5) followed by an erosion operation (equation 3.6).

$$
\begin{aligned}
& (A \ominus \mathrm{B})(\mathrm{x}, \mathrm{y})=\min \left\{A\left(x+x^{\prime}, y+y^{\prime}\right) \mid\left(x+x^{\prime}\right),\left(y+y^{\prime}\right) \in D_{A,},\left(x^{\prime}, y^{\prime}\right) \in D_{B}\right\} \\
& (A \bigoplus \mathrm{B})(\mathrm{x}, \mathrm{y})=\max \left\{A\left(x-x^{\prime}, y-y^{\prime}\right) \mid\left(x-x^{\prime}\right),\left(y-y^{\prime}\right) \in D_{A,},\left(x^{\prime}, y^{\prime}\right) \in D_{B}\right\}
\end{aligned}
$$

where $D_{A}$ is the domain of the structuring element $\mathrm{A}, D_{B}$ is the domain of the image $\mathrm{B},\left(x^{\prime}, y^{\prime}\right)$ are the coordinates of the flat structuring element and $(x, y)$ is the coordinate of a pixel of interest in the image A. As the diameter of the disc is larger than the width of the vessels and the major axis length of the leakage, after the opening operating, in the resulting image the background maintains approximately the same intensity values. The regions of the blood vessels and leakage became with intensity values comparable to the background. So, in the resulting image from the subtraction of "opened" image from the original one, the background pixels have values close to 0 and the pixel vessels values decrease a bit.

In M. S. El-Shahawy et al. [26], the region of analysis is considered to have decreasing pixel intensities values to the center (macula). A Gaussian surface can be used to model this intensity variation. Two Gaussian functions were used because one Gaussian function was not totally sufficient in some abnormal cases.

A. S. Fahmy et. al. [27] modeled the image intensity with gamma functions (equation 3.7) [27].

$$
I(t)=A\left(t^{\alpha} \cdot e^{-\frac{t}{\beta}}\right)
$$

where $A$ represents the peak amount of fluorescein that accumulates in a circulation of dye, $\alpha$ and $B$ are arbitrary parameters found with Levenberg-Marquardt Least Square method [40] and $\mathrm{t}$ is the acquisition time of the image. Indeed, A. S. Fahmy et. al. [27] performed a number of convolutions of gamma functions also dependent on the number of dye fluorescein circulations, i.e., one gamma function models the first circulation of fluorescein in the retina while the convolution of two gamma functions models the second circulation. As the FA exam typically covers a time interval of three circulation of the dye (10 $\mathrm{min})$, three gamma functions were required.

\subsubsection{Saliency Detection}

Y. Zhao et al. (2015) [24] applied an intensity cue [24] and a Retinex correction of inhomogeneity [41] for estimating the saliency map for the FA images. 
In Y. Zhao et al. (2016) [25], a superpixel approach was firstly employed to divide the image into meaningful patches at different levels. The saliency map of each individual superpixel at each level was estimated with two saliency cues, intensity [25] and compactness [25]. The saliency maps at different levels over the same cues were fused using an averaging operator. The final saliency map also conjugated the two-saliency maps over different cues with a pixelwise multiplication operator.

\subsubsection{Segmentation in Image Subtraction Approaches}

In H. Rabbani et al. [9], before segmentation, the mean-early-FA frames are subtracted from the late-FA frames. An opening operation utilizing a disk-shaped structuring element (with a radius of 2 pixels) was used to remove the small remaining vessels. Afterwards, by inpainting the removed vessels (previous step) and with dilation, followed by eroding the image using a disk-shaped structuring element (with radius of 5 and 3 pixels), it was possible to maintain the leakage areas that overlap vessels. Finally, Chan-Vese's active contour segmentation algorithm [33] was used to locate the leak.

In M. S. El-Shahawy et al. [26], the subtraction of the mean early time frames from the mean late frames and a Gaussian Mixture Model (GMM) [42] allowed the segmentation. GMM using expectation maximization was used to segment the images into 2 classes: background and lesion. Lesions were considered as the pixels belonging to the class with the highest mean.

Segmentations based on region growing could also work well, since the subtraction itself allows a good approximation of the location of the lesions.

\subsubsection{Segmentation in Classification Approaches}

Classification-based segmentation algorithms assume that each image consists of different elements that present unique and distinct features throughout the angiographic sequence and this allows to assign different classes to these elements, i.e., the FA sequence in the leak region has unique properties compared to other regions of the image.

In C. Tsai et al. [28], the system used the intensity variation of spatially-corresponding pixels across the angiographic sequence with the targeted leakage pattern marked by an ophthalmologist. The learning strategy was Adaboost [43] and the intensity variation of the dye leak has 12 features: the average and the difference between intensity pixel values in 5 different time intervals and the slope and intercept of the straight line from linear least-squares regression of the time-intensity data points from the entire sequence. The result of Adaboost classification was a severity map for the sequence (each point in the global space was assigned a degree of severity). Lesions were segmented using Random Walker [44] algorithm initialized using seed points of very high or very low severity as leaks or background points, respectively. The probability that the random walker of each pixel first arrives at a seed with each label is 
calculated. After computing these probabilities for each pixel, the pixel is assigned to the label for which it is most likely to send a random walker.

A. S. Fahmy et al. [27] extracted three features of each coordinate of the image along the FA sequence to classify the FA image into three types: background, occult, and classic lesions. Classic lesions are those that appear from the beginning and the occult are shown as time passes. Vessels were ignored because the zone of analysis was not the whole image and the manually selected zones were considered without any vessel occurrence. The first feature was the time-to-peak intensity, i.e., the acquisition time for which the maximum intensity of each pixel was achieved. The lesion region presents a maximum intensity for each pixel in later times of the exam. The second feature was the pixel intensity of the second circulation of the dye (near 4 minutes after dye injection). In the background, pixel intensities of the second circulation are low. A third feature was the normalized difference between the intensity of the first (near 1 minute after dye injection) and the third circulation of the dye (near 7 minutes after dye injection). The difference is large in background regions. Unsupervised fuzzy C-means classifier was used to automatically achieve the classification.

\subsubsection{Segmentation in Saliency Approaches}

In Y. Zhao et al. [24] and in Y. Zhao et al. [25], a threshold was applied in the saliency maps to achieve a binary image which essentially contains the leak. Other anatomical structures like vessels and optic disc previously determined were set to 0 and any small and/or isolated objects were eliminated using a disk-shaped opening operation (with a radius of 2 pixels). The remaining regions corresponded to leakage areas.

\subsubsection{Evaluation}

The automatic segmentation is frequently compared with manual segmentation to access the performance of the algorithm. Accuracy (Acc) (equation 3.8), specificity (SN) (equation 3.9) and specificity (SP) (equation 3.10) are the statistical measures which are most commonly used to evaluate performance.

$$
\begin{gathered}
A c c=\frac{(T P+T N)}{(T P+T N+F P+F N)} \\
S N=\frac{T P}{(T P+F N)} \\
S P=\frac{T N}{(F P+T N)}
\end{gathered}
$$

where the true positive (TP) is the number of pixels classified as leak in the ground truth and in the segmented image, the true negative (TN) is the number of pixels identified as a non-leak in the ground truth and in the segmented image, the false negative (FN) is the number of pixels 
marked as non-leak in the segmented image but as a leak pixel in the ground truth image and the false positive (FP) is number of the segmented image pixels that are in the leak but are in the background in the ground truth image.

Accuracy is calculated by the ratio between the total number of correctly classified pixels and the number of pixels in the image. On one hand, sensitivity reflects the ability to segment the leak pixels, on the other hand, specificity is the capability to detect non-leak pixels.

In addition, the performance of the algorithm can also be measured with the area under the receiver operating characteristic (ROC) curve. The ROC curve is plot of the true-positive fraction versus the false-positive fraction for different cut-off points of a parameter.

The sensitivity and the specificity are calculated in M. S. El-Shahawy et al. [26]. In C. Tsai et al. [28] only the accuracy is present. On other hand, in $H$. Rabbani et al [9], the previous three metrics are available. In Y. Zhao et al. (2015) [24] and Y. Zhao et al. (2015) [25], the area under the curve (AUC) is a metric calculated in addition to the previous ones.

\subsubsection{Limitations}

All the described algorithms allow the segmentation of lesions in the last frame of the sequence. In this dissertation work, the segmentation of the remaining images of the angiographic sequence is also desired. Through the contours of the leaks of the last frame, an active contour segmentation algorithm can potentially be a solution to fulfill the segmentations of the other frames.

Although the studies in this section have shown some good results (table 3.1), they have some important limitations. In H. Rabbani et al [9] and M. S. El-Shahawy et al. [26], the detection areas of leakage are achieved in a rectangular region centered at the macula and performance metrics have low values. A. S. Fahmy et al. [27] study does not allow the leakage characterization in the whole image too. Moreover, it uses unsupervised classification, so it is not very robust to the variations of image intensity and occurrences of lesions that overlap vessels. C. Tsai et al. [28] applied AdaBoost to classify the leakage regions of FA images. Although, with training, supervised classifiers may overcome some of the inherent challenges of the leakage characterization, they are extremely dependent on the good quality of the manual annotation. Y. Zhao et al. (2015) [24] only use the intensity information to generate the saliency map for the detection, which may suffer when some non-leakage areas also have high intensities. In Y. Zhao et al. (2016) [25], the parameters have been adjusted through tryand-error. 
Table 3.1 - Schematic/Summary representation of the state of the art for leakage segmentation (n.a. - not applicable).

\begin{tabular}{|c|c|c|c|c|c|c|c|}
\hline \multirow[b]{2}{*}{ Study } & \multirow[b]{2}{*}{ Approach } & \multicolumn{4}{|c|}{ Registration } & \multirow[b]{2}{*}{ Vessel detection } & \multirow[b]{2}{*}{ Optic disc detection } \\
\hline & & Feature extraction & Object & $\begin{array}{c}\text { Nature of } \\
\text { transformation }\end{array}$ & $\begin{array}{l}\text { Domain of } \\
\text { transformation }\end{array}$ & & \\
\hline \multirow{2}{*}{$\begin{array}{l}\text { H. Rabbani et } \\
\text { al. [9] }\end{array}$} & \multirow{2}{*}{$\begin{array}{l}\text { Comparative and with image } \\
\text { subtraction of mean early } \\
\text { frames from mean late frames }\end{array}$} & SURF method with MSAC & $\begin{array}{c}\text { Vessel } \\
\text { tree }\end{array}$ & Affine & Global & \multirow{2}{*}{$\begin{array}{c}\text { Dijkstra forest } \\
\text { algorithm of Estrada et } \\
\text { al. }\end{array}$} & \multirow[t]{2}{*}{ n.a. } \\
\hline & & Multi-resolution registration & Image & Non-rigid & Local & & \\
\hline $\begin{array}{c}\text { M. S. El- } \\
\text { Shahawy et al. } \\
{[26]}\end{array}$ & $\begin{array}{l}\text { Comparative and with image } \\
\text { subtraction of mean early } \\
\text { frames from mean late frames }\end{array}$ & Phase-correlation & $\begin{array}{l}\text { Vessel } \\
\text { tree }\end{array}$ & Rigid & Global & $\begin{array}{l}\text { Matched filters + Fuzzy } \\
\text { K-means classifier }(\mathrm{K}=3)\end{array}$ & n.a. \\
\hline $\begin{array}{l}\text { C. Tsai et al. } \\
\text { [28] }\end{array}$ & $\begin{array}{l}\text { Comparative and } \\
\text { classification-based }\end{array}$ & ED-DB-ICP & Image & Quadratic & Global & n.a. & n.a. \\
\hline $\begin{array}{l}\text { A. S. Fahmy et } \\
\text { al. [27] }\end{array}$ & $\begin{array}{l}\text { Comparative and } \\
\text { classification-based }\end{array}$ & Phase-correlation & Image & Rigid & Global & n.a. & n.a. \\
\hline $\begin{array}{l}\text { Y. Zhao et al. } \\
\text { [24] }\end{array}$ & Saliency-based & n.a. & n.a. & n.a. & n.a. & $\begin{array}{c}\text { Local phase } \\
\text { enhancement }+ \text { Chan- } \\
\text { Vese segmentation }\end{array}$ & $\begin{array}{l}\text { Region where more than } 5 \\
\text { vessels are crossed }\end{array}$ \\
\hline $\begin{array}{c}\text { Y. Zhao et al. } \\
{[25]}\end{array}$ & Saliency-based & n.a. & n.a. & n.a. & n.a. & IPACHR & $\begin{array}{l}\text { Region where more than } 5 \\
\text { vessels are crossed }\end{array}$ \\
\hline
\end{tabular}

\begin{tabular}{|c|c|c|c|c|}
\hline Study & $\begin{array}{l}\text { Modeling the background } \\
\text { intensity }\end{array}$ & Saliency detection & Complementary operations in the final segmentation & Leakage segmentation \\
\hline $\begin{array}{l}\text { H. Rabbani et } \\
\text { al. [9] }\end{array}$ & $\begin{array}{l}\text { Local histogram equalization + } \\
\text { top-hat filter (disk with radius } \\
\text { of } 20 \text { pixels) }\end{array}$ & n.a. & $\begin{array}{ll}\text { (i) } & \text { Vessels are substituted by surrounding mean pixel values } \\
\text { (ii) } & \text { Opening operation (disk with radius of } 2 \text { pixels) } \\
\text { (iii) } & \text { Inpainting of the removed vessels, dilation (disk with radius } \\
\text { of } 5 \text { pixels) and eroding the image (disk with radius of } 3 \text { pixels) }\end{array}$ & Chan-Vese \\
\hline $\begin{array}{c}\text { M. S. El- } \\
\text { Shahawy et al. } \\
{[26]}\end{array}$ & Gaussian functions & n.a. & Vessels are substituted by surrounding mean pixel values & GMM \\
\hline $\begin{array}{c}\text { C. Tsai et al. } \\
{[28]}\end{array}$ & n.a. & n.a. & n.a. & Random Walks algorithm \\
\hline $\begin{array}{l}\text { A. S. Fahmy et } \\
\text { al. [27] }\end{array}$ & Gamma functions & n.a. & n.a. & Classic and occult classes \\
\hline $\begin{array}{l}\text { Y. Zhao et al. } \\
{[24]}\end{array}$ & n.a. & $\begin{array}{c}\text { Intensity estimation of the } \\
\text { saliency map + Retinex } \\
\text { correction }\end{array}$ & $\begin{array}{l}\text { Vessels and optic disc are set to } 0 \\
\text { Opening operation (disk with radius of } 2 \text { pixels) }\end{array}$ & Threshold \\
\hline $\begin{array}{l}\text { Y. Zhao et al. } \\
\text { [25] }\end{array}$ & n.a. & $\begin{array}{c}\text { Super-pixel based } \\
\text { intensity and compactness } \\
\text { saliency maps }\end{array}$ & $\begin{array}{l}\text { Vessels and optic disc are set to } 0 \\
\text { Opening operation (disk with radius of } 2 \text { pixels) }\end{array}$ & $\begin{array}{c}\text { Threshold }(=0,65 \text {, scale } 0 \\
\text { to } 1)\end{array}$ \\
\hline
\end{tabular}




\begin{tabular}{|c|c|c|c|}
\hline \multirow{2}{*}{ Study } & \multicolumn{2}{|r|}{ Classification } & \multirow{2}{*}{ Performance metrics } \\
\hline & $\begin{array}{l}\text { Method for leakage } \\
\text { segmentation }\end{array}$ & Features & \\
\hline $\begin{array}{l}\text { H. Rabbani et } \\
\text { al. [9] }\end{array}$ & n.a. & n.a. & $\begin{array}{l}\text { Sensitivity: } 0,69 \pm 0,16 \\
\text { Specificity: } 0,91 \pm 0,09 \\
\text { Accuracy: } 0,86 \pm 0,08\end{array}$ \\
\hline $\begin{array}{l}\text { M. S. El- } \\
\text { Shahawy et al. } \\
\text { [26] }\end{array}$ & n.a. & n.a. & $\begin{array}{l}\text { Specificity: } 0,90 \\
\text { Sensitivity: } 0,70\end{array}$ \\
\hline $\begin{array}{l}\text { C. Tsai et al. } \\
\text { [28] }\end{array}$ & Supervised AdaBoost & $\begin{array}{l}12 \text { features: the intensity value and the intensity change in } 5 \text { time intervals }+ \text { the slope and intercept of } \\
\text { the straight line from linear least-squares regression of the time-intensity data points from the entire } \\
\text { sequence }\end{array}$ & Accuracy: 0,751 \\
\hline $\begin{array}{l}\text { A. S. Fahmy et } \\
\text { al. [27] }\end{array}$ & Unsupervised fuzzy C-means & $\begin{array}{l}3 \text { features: the time-to-peak intensity, the intensity of the second dye circulation and the normalized } \\
\text { difference between the intensity of the first and the third circulations of the dye }\end{array}$ & n.a. \\
\hline $\begin{array}{l}\text { Y. Zhao et al. } \\
{[24]}\end{array}$ & n.a. & 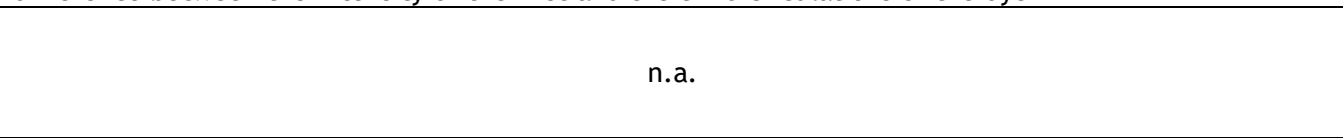 & $\begin{array}{c}\text { Sensitivity: } 0,808 \\
\text { Specificity: } 0,821 \\
\text { Accuracy: } 0,804 \\
\text { AUC: } 0,815\end{array}$ \\
\hline $\begin{array}{l}\text { Y. Zhao et al. } \\
\text { [25] }\end{array}$ & n.a. & n.a. & $\begin{array}{c}\text { Sensitivity: } 0,93 \pm 0,03 \\
\text { Specificity: } 0,96 \pm 0,02 \\
\text { Accuracy: } 0,94 \pm 0,02 \\
\text { AUC: } 0,82 \pm 0,03\end{array}$ \\
\hline
\end{tabular}




\section{Chapter 4}

\section{Methodology}

\subsection{Materials}

The training set and the test set, respectively, have seven and three fluorescein angiographic sequences from different patients (table 4.1). The number of images, the acquisition times, and the number of leaks in each sequence are variable. The image resolution between sequences is also different.

The implementation of the algorithm for the characterization of CSC lesion in FA images was performed in MATLAB R2016a with image analysis and computer vision toolboxes.

The images were acquired with a SPECTRALIS $®$ scanning laser angiography produced by Heidelberg Engineering. For workflow efficiency and diagnostic precision, FA (left part of figure 4.1) and ICGA (right part of figure 4.1) can be acquired simultaneously. SPECTRALIS scanning laser angiography can often be performed with less dye than the traditional fundus cameras, which may reduce the risk of allergic reactions and patient discomfort. Although some dataset sequences present both FA and ICGA images, such as in figure 4.1, in this work only FA images are used. Indeed, fluorescein angiography allows a good visualization of the leaks. A larger area of the retina is photographed, if the field of view (FoV) increases. Turning the sensitivity knob increases or decreases the light sensitivity of the camera into the ART (Automatic Real-Time mode). 
Table 4.1 - Acquisition times of different image series.

\begin{tabular}{|c|c|c|c|c|c|c|c|c|c|c|c|c|}
\hline \multirow{2}{*}{\multicolumn{3}{|c|}{$\begin{array}{l}\text { Dataset } \\
\text { Series }\end{array}$}} & \multicolumn{7}{|c|}{ Train } & \multicolumn{3}{|c|}{ Test } \\
\hline & & & $B$ & C & $\mathrm{F}$ & $G$ & $\mathrm{H}$ & $M$ & $\mathrm{~S}$ & $\mathrm{~A}$ & $\mathrm{D}$ & $\mathrm{E}$ \\
\hline \multirow{37}{*}{$\dot{i}$} & $\begin{array}{ll} \\
\end{array}$ & \multirow{37}{*}{ 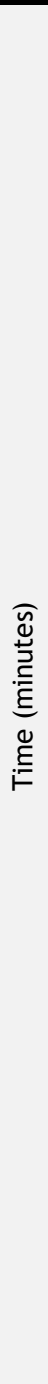 } & 0.69 & 0.36 & 0.43 & 1.14 & 0.42 & 0.43 & 1.97 & 0.36 & 0.59 & 0.50 \\
\hline & 2 & & 0.73 & 0.39 & 0.49 & 1.20 & 0.47 & 0.49 & 2.06 & 0.39 & 0.79 & 0.53 \\
\hline & 3 & & 0.90 & 0.41 & 0.51 & 1.29 & 0.52 & 0.54 & 2.17 & 0.42 & 0.85 & 0.94 \\
\hline & 4 & & 1.10 & 0.42 & 0.53 & 1.44 & 0.54 & 0.65 & 2.26 & 0.45 & 1.36 & 2.03 \\
\hline & 5 & & 1.64 & 0.47 & 0.55 & 1.51 & 0.56 & 0.83 & 2.28 & 0.47 & 2.45 & 3.70 \\
\hline & 6 & & 1.72 & 0.49 & 0.57 & 1.58 & 0.58 & 1.00 & 2.38 & 0.50 & 2.86 & 5.19 \\
\hline & 7 & & 2.10 & 0.54 & 0.59 & 1.67 & 0.60 & 1.06 & 2.41 & 0.52 & 4.11 & 6.02 \\
\hline & 8 & & 2.25 & 0.57 & 0.62 & 1.79 & 0.62 & 1.20 & 2.46 & 0.55 & 4.93 & 9.96 \\
\hline & 9 & & 2.76 & 0.60 & 0.67 & 1.90 & 0.64 & 1.70 & 2.49 & 0.57 & 5.67 & \\
\hline & 10 & & 3.04 & 0.69 & 0.69 & 2.01 & 0.73 & 1.97 & 2.52 & 1.07 & 7.11 & \\
\hline & 11 & & 3.52 & 0.74 & 0.76 & 2.29 & 0.75 & 2.12 & 2.54 & 1.11 & 10.30 & \\
\hline & 12 & & 5.43 & 0.87 & & 2.59 & 0.77 & 2.65 & 2.58 & 1.13 & & \\
\hline & 13 & & 5.52 & 0.93 & & 3.30 & 0.79 & 3.13 & 2.61 & 8.74 & & \\
\hline & 14 & & 6.52 & 0.99 & & 3.86 & 0.82 & 3.22 & 2.74 & & & \\
\hline & 15 & & 9.97 & 1.07 & & 4.08 & 0.84 & 3.62 & 3.79 & & & \\
\hline & 16 & & 10.04 & 1.10 & & 5.00 & 0.86 & 3.72 & 7.01 & & & \\
\hline & 17 & & & 1.54 & & 5.59 & 0.96 & 4.29 & 7.04 & & & \\
\hline & 18 & & & 3.34 & & 5.89 & 0.99 & 4.32 & 7.06 & & & \\
\hline & 19 & & & 3.36 & & 6.66 & 1.01 & 4.65 & 9.39 & & & \\
\hline & 20 & & & 3.37 & & 7.05 & 1.57 & 5.15 & 9.41 & & & \\
\hline & 21 & & & 3.39 & & 7.85 & 1.96 & 5.80 & 9.44 & & & \\
\hline & 22 & & & 3.52 & & 9.63 & 1.99 & & 9.47 & & & \\
\hline & 23 & & & 8.44 & & & 2.02 & & 9.51 & & & \\
\hline & 24 & & & 8.46 & & & 2.60 & & 9.57 & & & \\
\hline & 25 & & & & & & 2.63 & & 9.60 & & & \\
\hline & 26 & & & & & & 3.12 & & 9.66 & & & \\
\hline & 27 & & & & & & 3.14 & & 9.68 & & & \\
\hline & 28 & & & & & & 3.46 & & 9.70 & & & \\
\hline & 29 & & & & & & 3.91 & & 9.74 & & & \\
\hline & 30 & & & & & & 4.02 & & 9.76 & & & \\
\hline & 31 & & & & & & 4.76 & & & & & \\
\hline & 32 & & & & & & 5.15 & & & & & \\
\hline & 33 & & & & & & 6.59 & & & & & \\
\hline & 34 & & & & & & 7.52 & & & & & \\
\hline & 35 & & & & & & 8.42 & & & & & \\
\hline & 36 & & & & & & 8.49 & & & & & \\
\hline & 37 & & & & & & 10.06 & & & & & \\
\hline
\end{tabular}




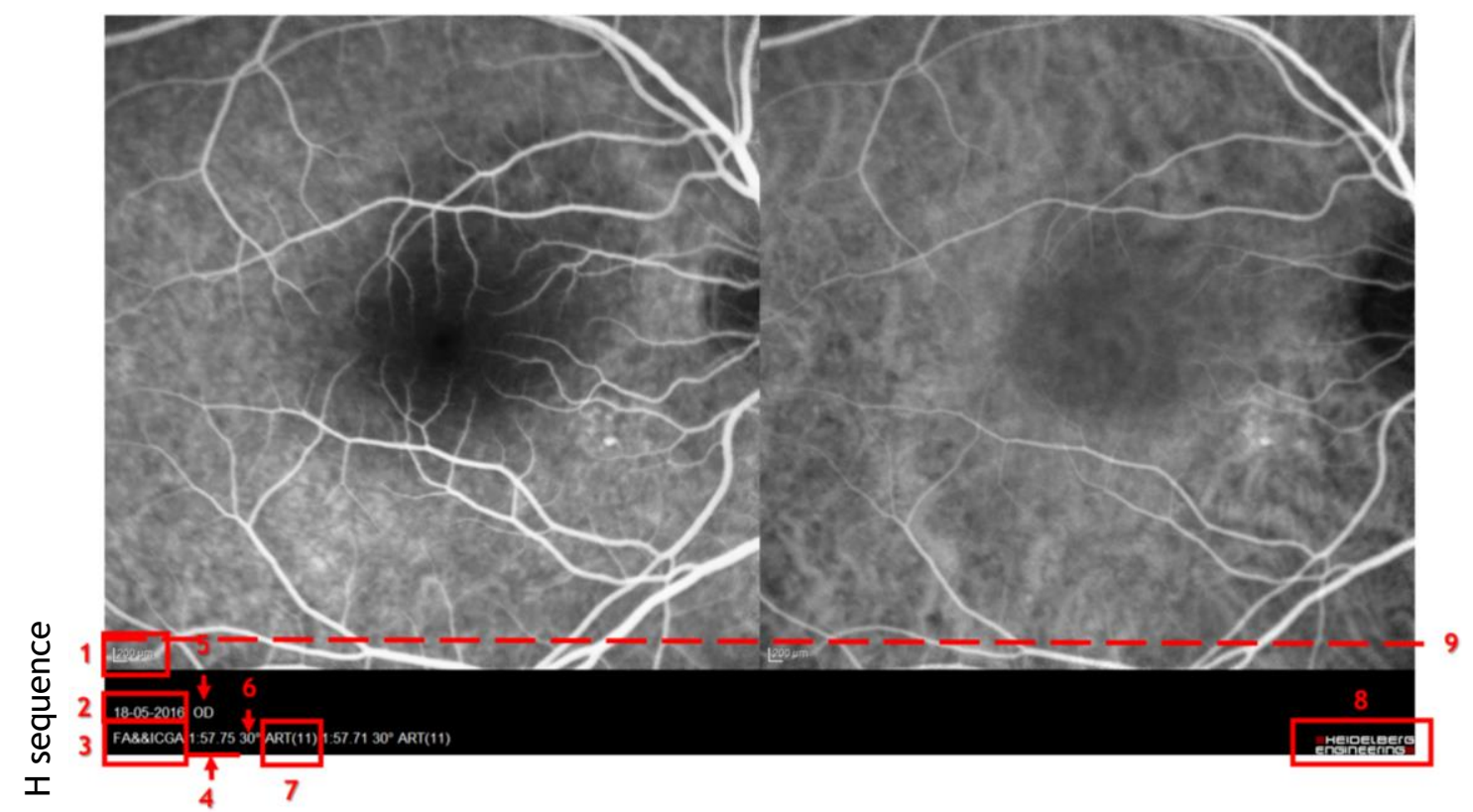

Figure 4.1 - FA and ICGA images acquired.

Label: 1 - scale; 2 - date; 3 - type of the acquired images; 4 - time (minute, second, hundredths of a second); 5 - eye; 6 - field of view; 7 - sensitivity knob; 8 - producer; 9 - dividing line between the image that is used for analysis (above) or the footer (below)

\subsection{Overview}

There are three possible approaches for segmenting leaks: comparative by image subtraction, comparative with (supervised or unsupervised) classification and through saliency detection. Comparative approaches are preferable to salient detection methods because they use temporal information. As the area of leaks grows throughout the examination, the temporal information can be useful in the detection and segmentation of the leaks. Nevertheless, the comparative methods with classification are not appropriate for this work. Approaches with supervised classifiers are very dependent of the amount of the data, and in this case the dataset is too small. They are also dependent of the quality of the manual annotation. Unsupervised classifiers are not robust for leakage characterization challenges. For these reasons, in this work, a comparative approach with image subtraction was chosen to quantitatively assess CSC leaks in angiographic sequences of retinal images. The subtraction approaches may overcome the challenges of image characterization until the final segmentation.

In figure 4.2, a schema of the proposed algorithm is represented. Table 4.2 summarizes which images are used in each step. 
Table 4.2 - Type of input images in each step of the implemented algorithm.

\begin{tabular}{|l|l|}
\hline \multicolumn{1}{|c|}{ Step } & \multicolumn{1}{c|}{ Type of input images } \\
\hline Frame selection & All the acquired frames \\
\hline Time detection & All the selected frames \\
\hline Image denoising & $\begin{array}{l}\text { The earliest selected frame serves as the target for all other selected } \\
\text { frames in the sequence }\end{array}$ \\
\hline Image registration & Earliest selected frame \\
\hline Vessel segmentation \\
$\begin{array}{l}\text { Candidate selection in } \\
\text { the early frames }\end{array}$ & Earliest selected frame \\
\hline Vessel inpainting & All the registered frames \\
\hline Optic disc detection & Earliest inpainted frame and the vessel tree \\
\hline Intensity normalization & Early and late inpainted frames \\
\hline $\begin{array}{l}\text { Background and optic } \\
\text { disc removal }\end{array}$ & Early and late inpainted frames \\
\hline Image subtraction & $\begin{array}{l}\text { The mean of the early frames after the background removal is subtracted } \\
\text { from the mean of the late frames after the background removal }\end{array}$ \\
\hline $\begin{array}{l}\text { Otsu method } \\
\text { Region growing } \\
\text { segmentation }\end{array}$ & frames by processed early frames \\
\hline $\begin{array}{l}\text { Active contour } \\
\text { segmentation }\end{array}$ & All the registered frames \\
\hline
\end{tabular}
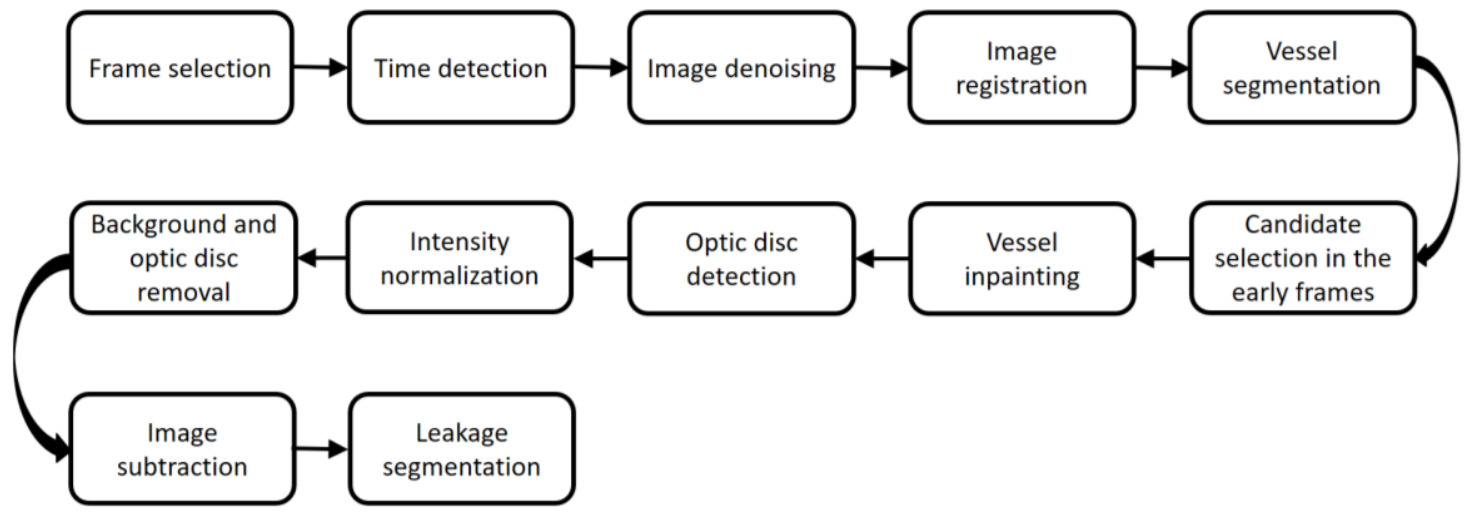

Figure 4.2 - Diagram representing the implemented process of the quantitative assessment of CSC leakages in sequences of FA images.

The proposed algorithm starts by the selection of images from the initial sequence. As mentioned in chapter 3.1, the suitable images to identify the leaks are those in which the retinal anatomical structures are fully distinguishable, i.e., the dye has fully covered these structures and has not yet lost fluorescence. Manual selection of sequence of angiographic sequence was the selected method.

The following step is frame time detection. As one of the work objectives is to analyze the growth of the dye leakage during the examination, the acquisition time of each frame, which can be obtained through the information present in the image footer, needs to be extracted. As the acquisition time always appears in the same position in the image, the recognition was achieved using optical character recognition [45, 46]. In addition, the video frames are automatically cropped to exclude irrelevant data (as the date, the magnification scale and producer). In this way, the useful part remains without the footer, facilitating the processing in later stages of the algorithm. 
The proposed method in this work requires image registration. As it is based on the subtraction of images, the same anatomical point needs to be in the same position over time. However, before image registration, image denoising are applied. Indeed, noise always affects the acquisition process so, Gaussian filters and median filters allows the improvement of the image quality. After that, image registration is performed. The first image of the selected set of the sequence serves as target image from the remaining ones. The detection and matching of the points in the images are achieved using the SURF methodology and SSD, respectively, and afterwards allows a projective transformation to be applied to the source image.

As already mentioned, there are two types of leaks. They can be distinguished by time criteria given that some appear in early stages and other in mid stages, i.e., the first image of the sequence already contains the lesion in the first case unlike the second case. As the images are registered previously, the segmentation of blood vessels in the first image and replacement of these pixels by neighboring values (especially background) in all images of the sequence allow the leaks to become mostly the regions of the image with highest intensities. However, as leaks may appear in the first images and vessel segmentation may include these regions because both have similar pixel intensities, an intermediate step before the vessel inpainting was introduced to try to determine the lesions and discard them in the mask of blood vessels. The lesions appear to have a circular shape in the first images in which they are visible so a blob detection method was used to detect the potential lesions. More specifically the method described in [35] was used for blood vessel segmentation and the sliding band filter (SBF) [47] was used as a blob detector to determine potential regions with lesion in the first image of the sequence.

Since the optic disc may have late staining, it also needs to be detected and its influence reduced. The optic disc has been detected based on three conditions: first, as a region of great concentration of blood vessels; secondly, because it is a region where the inclination of blood vessels is close to $0^{\circ}$; and finally, it is a region darker than the background in the early images of the sequence.

There are late frames where the leakage intensities are very low so even after background removal, these regions can have intensity values very similar to processed background regions. Therefore, image intensity normalization is performed prior to background removal in order to try to estimate the intensity of the background. In addition, image intensity normalization allows the intensity of the leaks to increase in cases of low luminosity, or that it decreases in cases of high luminosity. The normalization process uses well distributed and well-matched points from the first image and another source image of the sequence to process the intensity fit through a second-degree polynomial in the source image. Finally, the background was removed, which also reduces the effects of luminosity on the correct detection of leaks. Background removal is reached with morphological filters (top-hat) and the region containing the optic disc is replaced by 0 's after removing the background in all images. 
As the influence of all retinal structures is reduced in the steps described before, the subtraction between the mean of the late images and the mean of the early images allow good detection of regions that increase in intensity throughout the sequence. If the sequence contains leaks, the histogram of this subtraction images would be biomodal. The peak of the first class corresponds to background pixel intensities and noise, while the second corresponds to leakage pixel values. With the Otsu method is possible get the region that leads to leaks but, as the mean of the late frames is performed, this region does not fully correspond to the lesion, so region growing is used to segment the total area corresponding to the leak in the last image of the sequence. The contour of the leak of the remaining images of the sequence is obtained through an algorithm of active contours with the leak contour of the frame immediately later. The quality of the segmentation is evaluated through the sensitivity, Jaccard index and Dice similarity coefficient.

At last, and through segmentation of images over time, the growth of lesions and the variation of their intensity is analyzed.

\subsection{Frame Selection}

As the method proposed for this work is based on the different properties that distinct anatomical structures present in the angiographic sequence overtime, the selection of reliable images becomes even more important. The time that fluorescein takes to fill the blood vessels and time it begins to lose fluorescence vary slightly in each human organism. On the other hand, the intensity of the images can vary considerably along the sequence. Therefore, a method of manual selection of frames was chosen, discarding the selection methods based by the acquisition time of each image and methods based on the differences of intensities between the images. The user also makes the choice of the early frames and the late frames. Manual selection allows a great security in the choice of the appropriate images. In figure 4.3, some inadequate and adequate sequence images suitable for applying the proposed method are shown. 


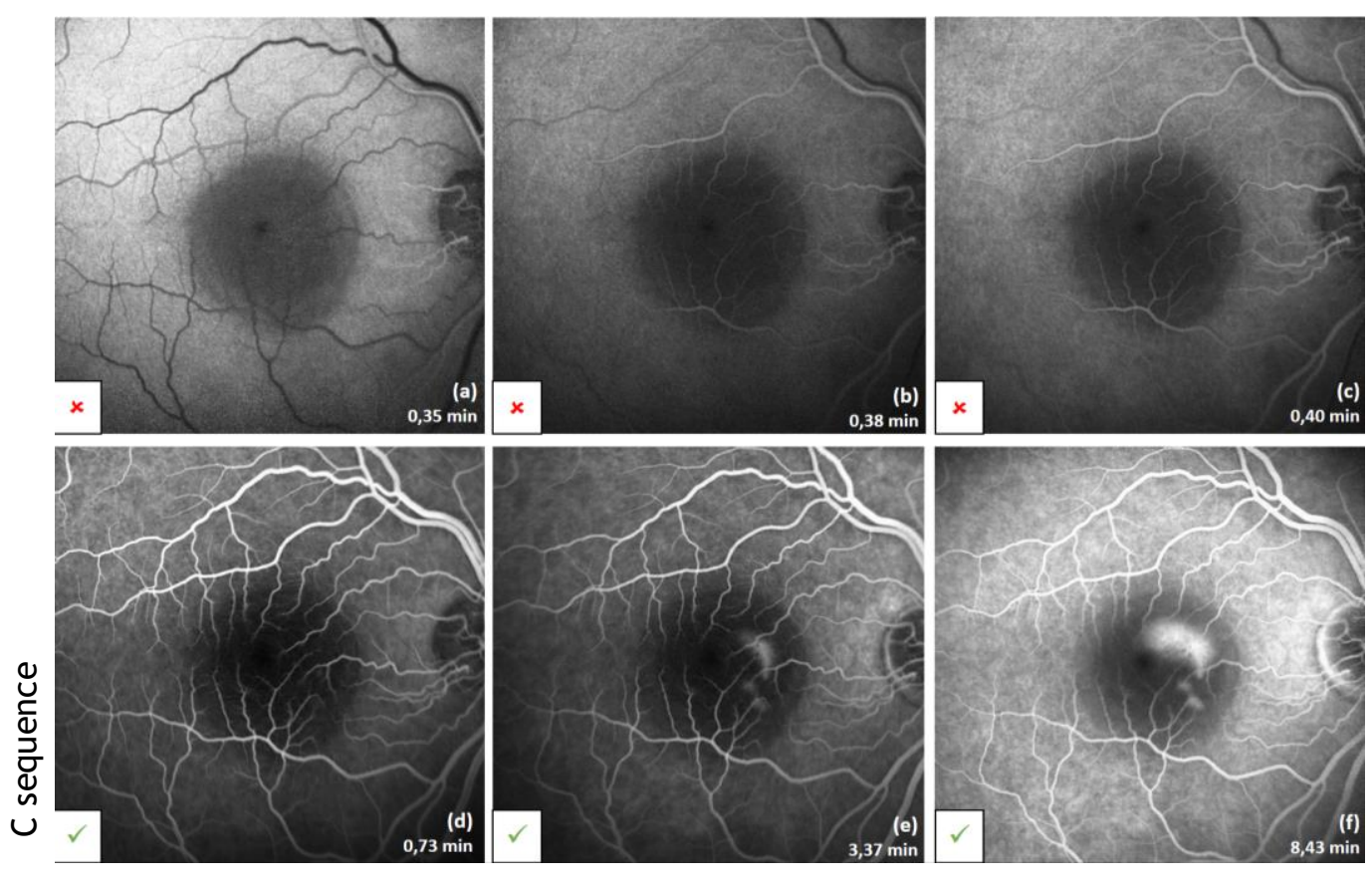

Figure 4.3 - Example of frame selection in an angiographic sequence (selected images represented by $\checkmark$, and discarded images represented by $x$ ).

\subsection{Time Detection}

In the algorithm for recognizing the time of image acquisition is discovered, i.e., the time since fluorescein was injected into the arm or hand. Time recognition is applied to all manually selected images. The algorithm used for this purpose is the optical character recognition (OCR) $[45,46]$ available in Matlab. The main steps of the OCR are preprocessing and character classification (static and adaptive).

Preprocessing techniques include registration, despeckle, binarization, line removal, layout analysis, aspect ratio normalization, scale normalization and character isolation. Registration is important when the lines, which contain the characters, are not perfectly vertical/horizontal. Despeckle techniques allow to remove some spots. Line removal cleans up non-glyph boxes and lines and layout analysis identifies regions with characters in columns, paragraphs, and tables.

Regarding the recognition itself, the first step includes a pass through a static classifier based on a decision tree. Every character successfully matched by the static classifier is sent into the adaptive classifier for training. The training is essential because the characters shapes recognized with high confidence on the first pass are used to recognize better the remaining letters on this second pass [45][46]. Feature extraction decomposes characters into "features" like lines, closed loops, line direction and line intersections. These features are compared with stored features of all representation of characters, with K-nearest neighbor algorithm [48], which might reduce to one or more character prototypes. 
Although FA images may have different resolutions and may come aggregated with ICGA images, the location of the part related to the acquisition time can be predicted. In this way, the OCR algorithm is only applied in the region where this information is supposed to exist (figure 4.4).

The field of view is immediately to the right of the time field at the footer of the image. In this type of images, the field of view has a value of $30^{\circ}$. Field of view characters are also recognized to allow better security in number detection thus, they work as control characters. Moreover, the time characters must always have between 5 and 6 numbers (if the time is less than or greater than 10 minutes respectively).
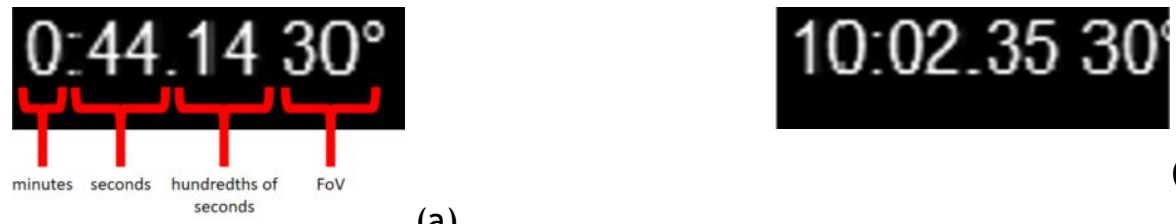

(a)

(b)

Figure 4.4 - Time recognition for less than (a) and greater than (b) 10 minutes.

The frames are cropped to exclude the footer in the remaining steps of the algorithm. After cropping, with the purpose of reducing computational time, the images are rescaled to a height of 768 pixels. The loss of resolution does not affect any step of the algorithm.

\subsection{Image Denoising}

The image denoising techniques are applied to all manually selected images. Some images appear to have salt \& pepper noise, with distributed black and white points. This is visible in the borders of the image. To eliminate this type of noise a $3 \times 3$ median filter is used.

Image acquisition is also affected by Guassian noise. This noise can be reduced using a spatial filter, though when smoothing an image, an undesirable outcome may result is the blurring of fine-scaled image edges and details because they also correspond to reduce high frequencies fluctuations. In this case, Gaussian smoothing (equation 4.1) was applied.

$$
g(x, y)=\frac{1}{2 \pi \sigma^{2}} e^{-\frac{x^{2}+y^{2}}{2 \sigma^{2}}}
$$

where $g(x, y)$ is the Gaussian function, $x$ is the distance from the central pixel of the filter in the horizontal axis, $y$ is the distance from the central pixel of filter in the vertical axis, and $\sigma$ is the standard deviation of the Gaussian distribution. The standard deviation is 0.5 and the kernel size is $3 \times 3\left(4^{*} \sigma+1\right)$. Figure 4.5 shows the image before and after the application of denoising methods. 


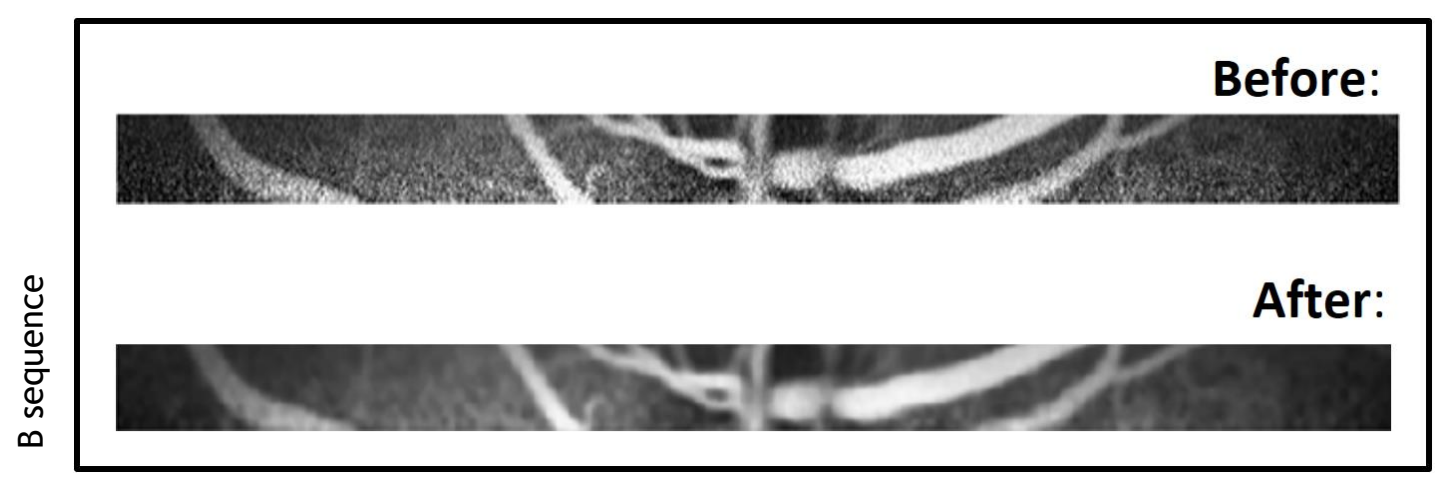

Figure 4.5 - The border of the image before and after the application of denoising techniques.

\subsection{Registration}

The steps of registration are (figure 4.6): point detection and feature extraction (with SURF methodology), matching (with SSD), wrong match elimination (with distance criteria and MSAC methodology) and geometric transformation (projective transformation is applied). The registration is applied between the first image of the sequence (target) and the remaining images of the sequence (source images).
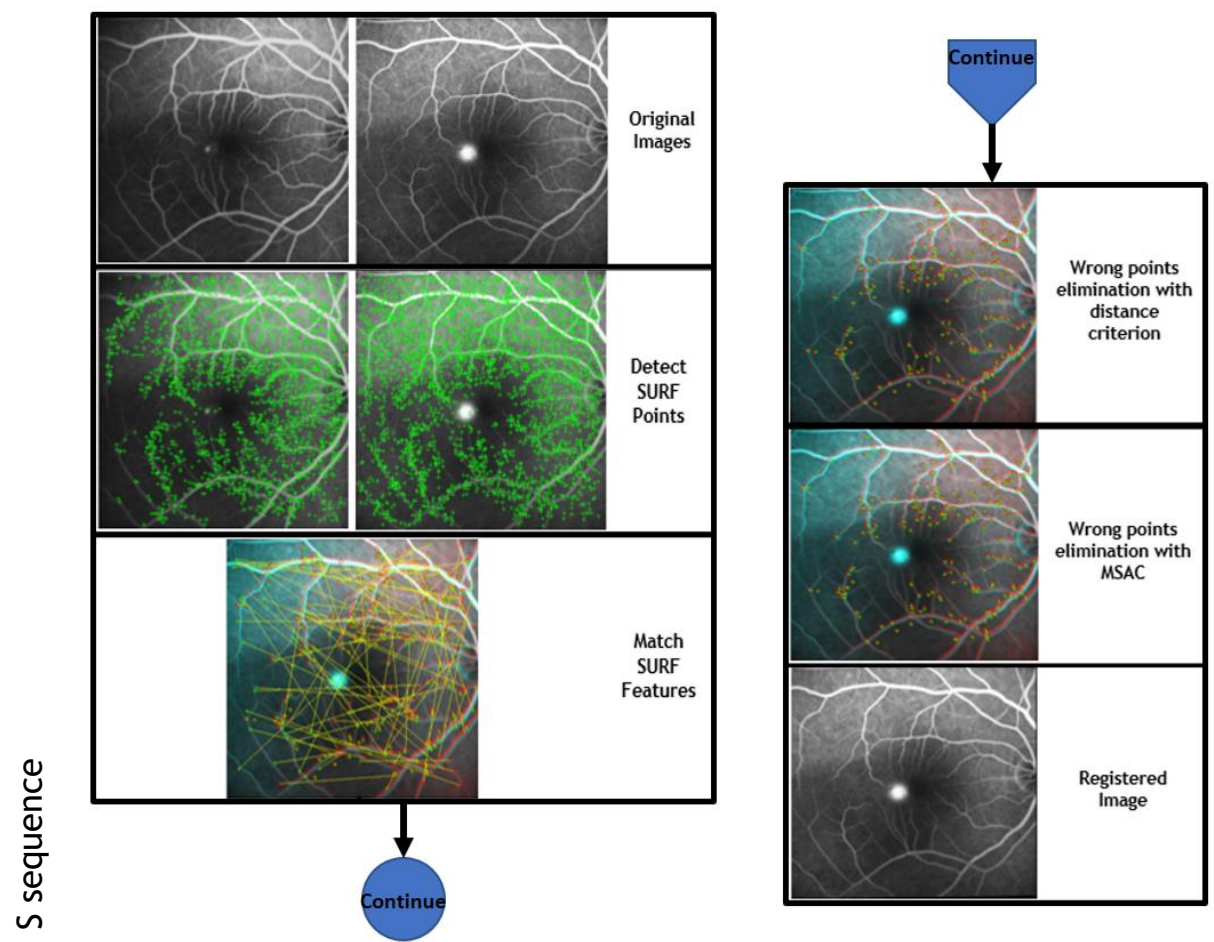

Figure 4.6 - Example of implementation of the registration developed. 
The registration was performed with a feature based approach because it has some advantages when compared with the use of intensity methodologies, such as the low computation cost and the independence of luminosity, rotation, and scaling [49].

The SURF methodology was used as a feature detection and description method in the whole image because it is a fast and invariant strategy for detecting blobs. So, it catches vessels bifurcations. Furthermore, it was described as a better methodology than other local feature detectors for retinal images [50]. The SURF methodology can be summarized in the following steps:

- Feature detection: SURF uses determinants of a modified Hessian matrix to locate image's significant points. The convolution of the integral image with approximated Gaussian kernels second order derivatives replace second order partial derivatives of the Hessian matrix $[29,51]$. The value of each pixel of the integral image is equal to the sum of all the pixel values above, to the left and including the target pixel itself of the original image;

- Scale-space representation and location of points of interest: To achieve scale invariance, algorithm searches for significant points on multiple image scale levels. Instead of replacing the image size, it changes the size of the convolution kernel (which depends of octaves). After convolution in the image with different octaves, the comparison of the responses of convolution between neighborhoods allow the return of the greatest variations;

- Orientation assignment: SURF makes points also rotation invariant. Given the position of a point of interest, an area around the point proportional to the scale, is set to the convolution (Haar wavelet responses) applied using two kernels (figure 4.7). For each pixel belonging to a region, the convolution response of the $x$-axis and the $y$-axis is obtained, so these two values are used and represent a point on a unit circle. The sector in the circle with the highest number of points is chosen and the main direction of the feature point is estimated by calculating the sum of all responses within the sector;

- Descriptor based on the sum of Haar wavelet responses: New windows are formed around the points, which have 20 times the size of the scale at which the point was found. The window is divided into parts 64 or 128 where the grouping of 4 parts form a sub window. The axes are aligned with the main direction of the point of interest [52]. The output values for each sub-window correspond to: 1 ) the sum of wavelet responses in the direction of the $\mathrm{x}$-axis, 2) module of the sum of wavelet responses in the direction of $x$-axis, 3) the sum of wavelet responses in the direction of the $y$-axis, 4) module of the sum of wavelet responses in the direction of the $y$ axis [53]. 


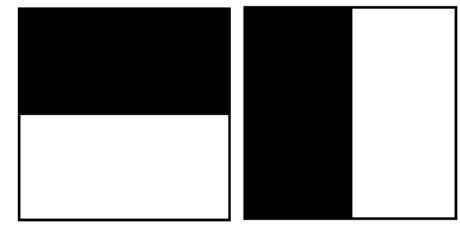

Figure 4.7 - Haar wavelet filters to compute the responses in $x$ (left) and y (right) [back regions and white regions are equal to -1 and 1 respectively].

In feature matching, the feature space distance with SSD was computed between each feature in the target image and the source image. For each feature in the target image, the most similar feature in source image was found. The strongest pairs of points are selected and the unambiguous correspondences are discarded.

The distances between the true matching points in two images are similar. So, with a histogram of distances between the points, a predominant peak must exist in this graphic. Then, pairings selection by distance criteria was used, the predominant peak is detected and only the points whose correspondences distances are between the lower and the upper limits are considered. The bins unitarily increase in width in each iteration until a predominant peak is found (more than $75 \%$ pairs covered).

After the distance criteria, MSAC allows to further filter the pairs. In MSAC cost function there is an inlier penalty based on the deviation from the required functional relation while outliers score a constant penalty [54]. MSAC operates a hypothesize-and-verify framework: a minimal subset of the input data points is randomly selected and model parameters are estimated. This hypothesize and verify loop is repeated until the probability of finding a model with better support than the current best model falls below a predefined threshold (typically $1 \%-5 \%)$ and the percentage of inliers exceeds the user's indications or the number of iterations is too large [55].

Finally, the projective transformation was determined. Equation 4.2 is a generic projective transformation with eight degrees of freedom [56].

$$
\left[\begin{array}{c}
x^{\prime} \\
y^{\prime} \\
1
\end{array}\right]=c \cdot\left[\begin{array}{ccc}
h 11 & h 12 & h 13 \\
h 21 & h 22 & h 23 \\
h 31 & h 32 & 1
\end{array}\right] \times\left[\begin{array}{l}
x \\
y \\
1
\end{array}\right]
$$

where $(x, y)$ are the coordinates of a point in the source image; $\left(x^{\prime}, y^{\prime}\right)$ are the coordinates of a point in the target image; $c, h 11, h 12, h 13, h 21, h 22, h 23, h 31$ and h32 are variables of a projective transformation.

As the grid point in the target image typically do not coincide with another grid point in the transformed space, the pixel values of the transformed image on grid must be estimated. Linear interpolation was the method used in this work which performs the distance-weighted average of the four nearest pixel values to estimate a new pixel value.

A pyramidal study (in which the number of parameter combinations decreased and the number of images increased during each iteration) was carried out to determine the best 
adjustable parameters in the registration method. The parameters are: the metric threshold (for each pixel, determinants of the modified Hessian matrix greater than this threshold will be considered points of interest), the match threshold (percentage of the stronger selected pairs) and the maximum ratio (maximum tolerable ratio to be considered as inlier pair between the nearest feature distance and the second nearest feature distance of the source vector and the target vector). NMI was used to measure the similarity between the target and the registered images. NMI is a recommended parameter for situations where there are luminosity variations of the images over time as in this case [57].

Figure 4.8 (a) is a target image, figure 4.8 (b) is a source image and figure 4.8 (c) is the registered image. The quality of registration process is assessed with the mean of the image which was registered and the target image (figure 4.8 (d)) and mosaicism (representation of alternating blocks taken from two images - figure $4.8(e))$.
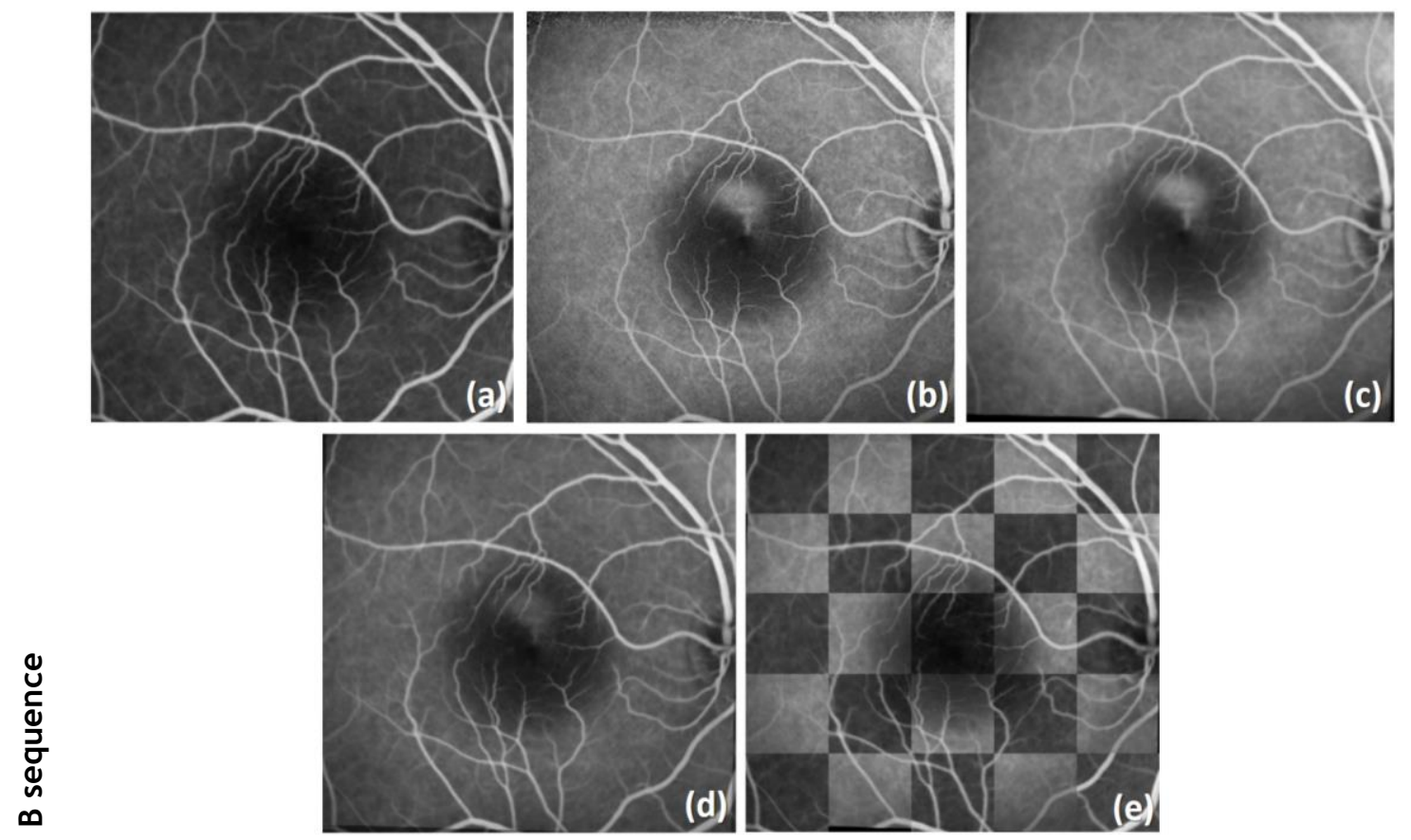

Figure 4.8 - Representative images of the registration process: target image (a), source image (b), registered image (c), mean of the registered image and the target images (d) and mosaicism between the registered image and the target image $(\mathrm{e})$.

\subsection{Vessel Segmentation}

The method proposed by Mendonça et al. [35] was used for segmenting the retinal vasculature. It was briefly described previously in section 3.2.3. In colour retinal fundus images, the algorithm uses the green channel to proceed to segmentation. FA images are grayscale, the entire image content is used and only one additional step has been introduced at the preprocessing level. In general, the method can be divided in three phases (figure 4.9): 
- Preprocessing: To deal with border effects, where normally nearby border pixels reduce their intensity, the original is padded with extra rows and columns. In this case, the regions up to 40 pixels from the borders of the image were reflected.

- Detection of the blood vessels centerlines: Centerlines candidates are detected with the information extracted from a set of four directional Difference of Offset Gaussian filters, then connected into parts by a region growing process, and, at last, the centerline segments are validated based on intensity and length properties;

- Final segmentation of vessels: Multi-scale morphological filters and Gaussian filters are used to enhance the regions belonging to vessels. Morphological reconstruction approaches are followed to generate binary maps of the vessels at different scales. The final image with the segmented vessels is obtained iteratively by combining the image with the centerlines with the set of images that resulted from the vessel reconstruction.
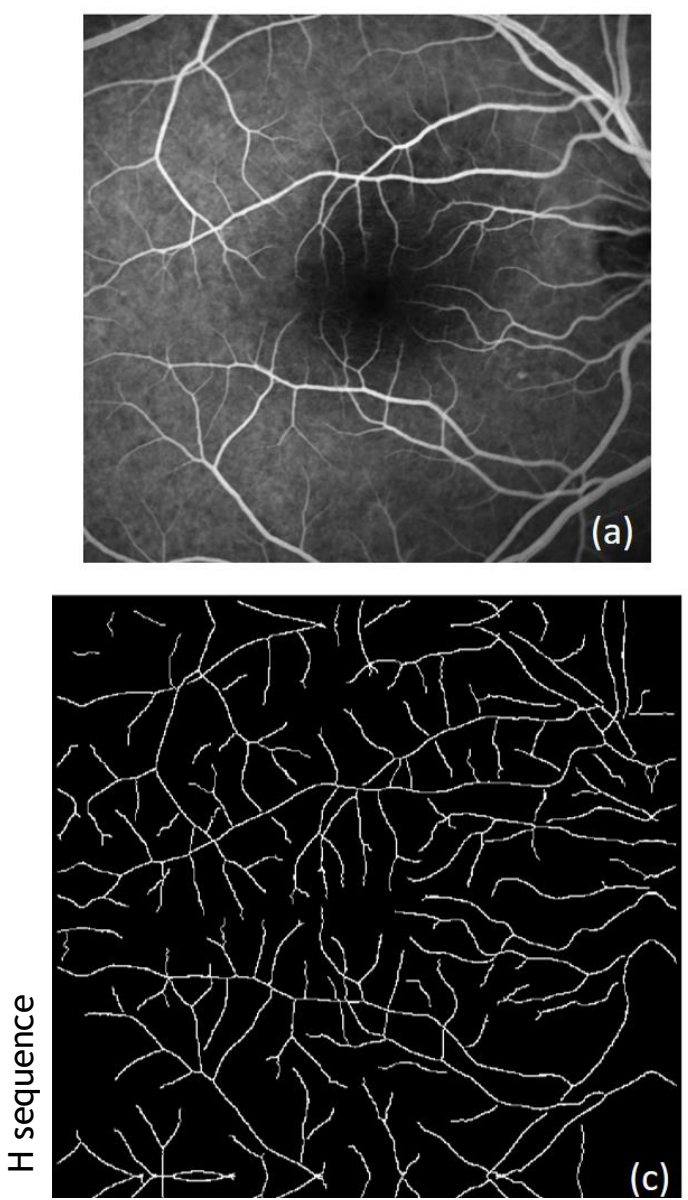
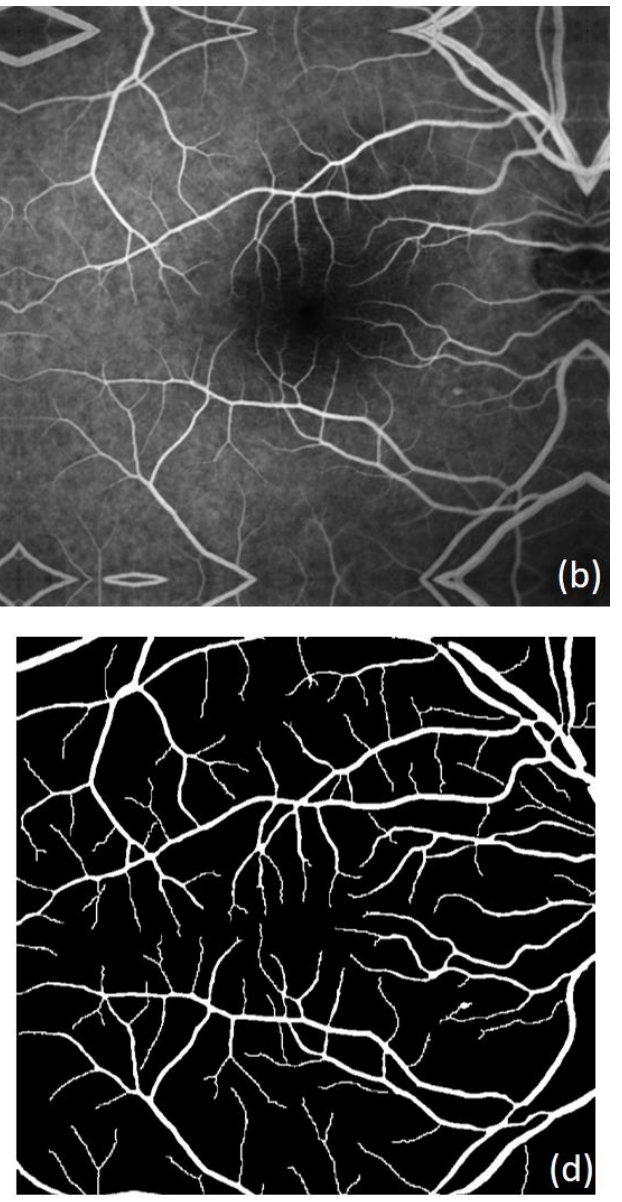

Figure 4.9 - Steps of the vessel segmentation algorithm: original image (a), preprocessing (b), detection of the centerlines (c) and final segmentation of vessels (d). 


\subsection{Candidate Selection in the Early Frames}

The first frame may or may not have leaks depending on the type of lesion. In any case, this situation should be considered. As the vascular network is used for the inpainting of blood vessels, the leak region cannot be present on the segmented binary map. In this way, circular regions (lesions shape in the first frame in which it is present) are searched in the image resulting from the earliest frame of the sequence with the binary vascular network image. The method for detecting these blobs is the sliding band filter [47]. Regions with radius greater than 10 and less than 30, the expected size of the leaks in the first image of the sequence, are searched.

The detected regions are removed from the vessels map (figure 4.10). Regions in vessels with high width can be potentially detected with the SBF because they have an intensity similar to the blood vessels. However, it is expected that, at the end, these regions will have no influence on the final segmentation since there is a subtraction of images in a later step so that the regions of constant intensity along the sequence are not considered as potential candidates for lesions.
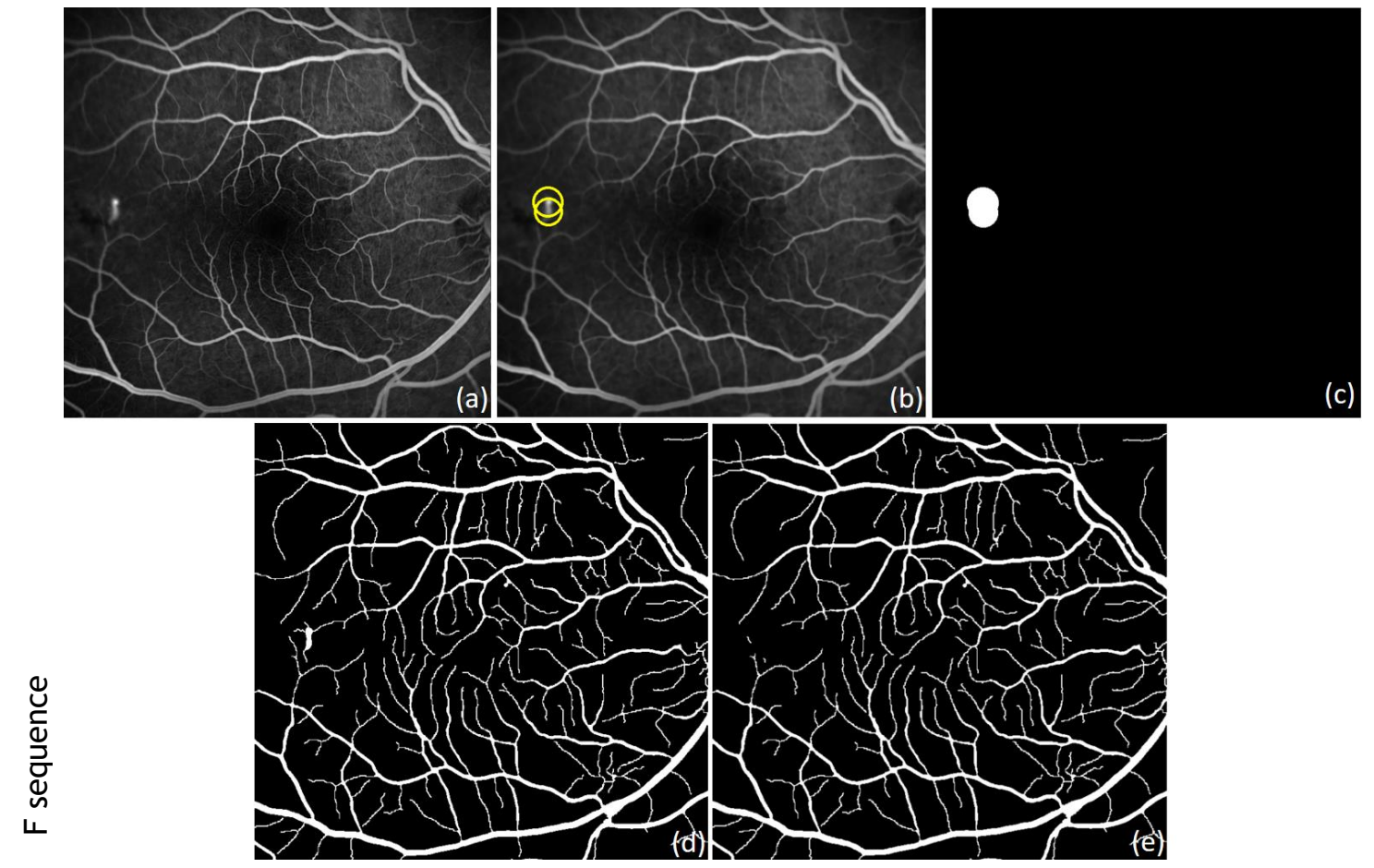

Figure 4.10 - Steps of the candidate selection in the early frames: original image (a), original image with detected regions (b), binary detected regions (c), image after vessel segmentation algorithm (d) and final binary vessel map used in the vessel inpainting (e).

The SBF is a convergence index $(\mathrm{Cl})$ filter and the SBF response at a pixel of a pixel of interest is defined by equations 4.3 and 4.4 . 


$$
\begin{aligned}
& S B F(x, y)=\frac{1}{N} \sum_{i=0}^{N-1} c_{i_{\max }} \\
& c_{i_{\max }}=\max \left(\frac{1}{d} \sum_{m=n}^{n+d} \cos \theta_{i, m}\right), n \in\left[R_{\min }, R_{\max }\right]
\end{aligned}
$$

where $\operatorname{SBF}(x, y)$ represents the filter response for each spatial coordinate $(x, y), c_{i_{\max }}$ is the convergence index for each region line, $N$ is the number of support region lines, $\theta_{i, m}$ represents the angle of the gradient vector at point $m$ pixels away from $P$ in direction $i, d$ corresponds to the width of the band, and $R_{\min }$ and $R_{\max }$ represent, respectively, the inner and outer sliding limits of the band. The SBF searches in each radial direction the band of fixed width that corresponds to the maximum degree of convergence, which implies that the position of this band is variable in each direction. Therefore, the output is a measure of the convergence degree of the gradient vectors calculated for the pixels belonging to the filter support region [58]. As the SBF has a more generic formulation that other $\mathrm{Cl}$ filters, it can be used to extract also elliptical regions. From the SBF filter response map, candidate regions are associated with the locations of filter maxima response, above a given threshold (in this case it is 0.8 ).

\subsection{Vessel Inpainting}

With the inpainting of blood vessels, leak segmentation turns easier since vessel regions, which have pixel intensities similar to the leaks, get lower intensities. The vascular segmentation used for this purpose is the one from the first image of the sequence (figure 4.11).

Due to sudden movements of the patients during the examination, some regions of the vessels would need elastic registration. In order to minimize these or other minor distortions, the mask of the blood vessels is dilated through a disk-shaped structuring element with a 4pixel radius. Each pixel that belongs to this mask is replaced by the mean of the pixels that do not belong to the mask, in a window of $13 \times 13$ pixels centered on the target pixel (TaP). Figure 4.12 represents the described window with the TaP, the mask pixels (intensity equal to 0 ) and the surrounding pixels (intensity greater than 0 ). Since only the pixels that do not belong to the mask are used, the mean of the neighborhood, which is the new pixel value, is 0.43 in the case of the figure 4.12. In the larger vessels, the size above mentioned may not be sufficient to include non-mask pixels. After the first inpainting of the vessels, if there are still pixels that belong to a region of vessels because they continue to have intensity value equal to 0 , then the window is increased to $25 \times 25$ pixels in these cases to ensure that they are also replaced by neighborhood values. 


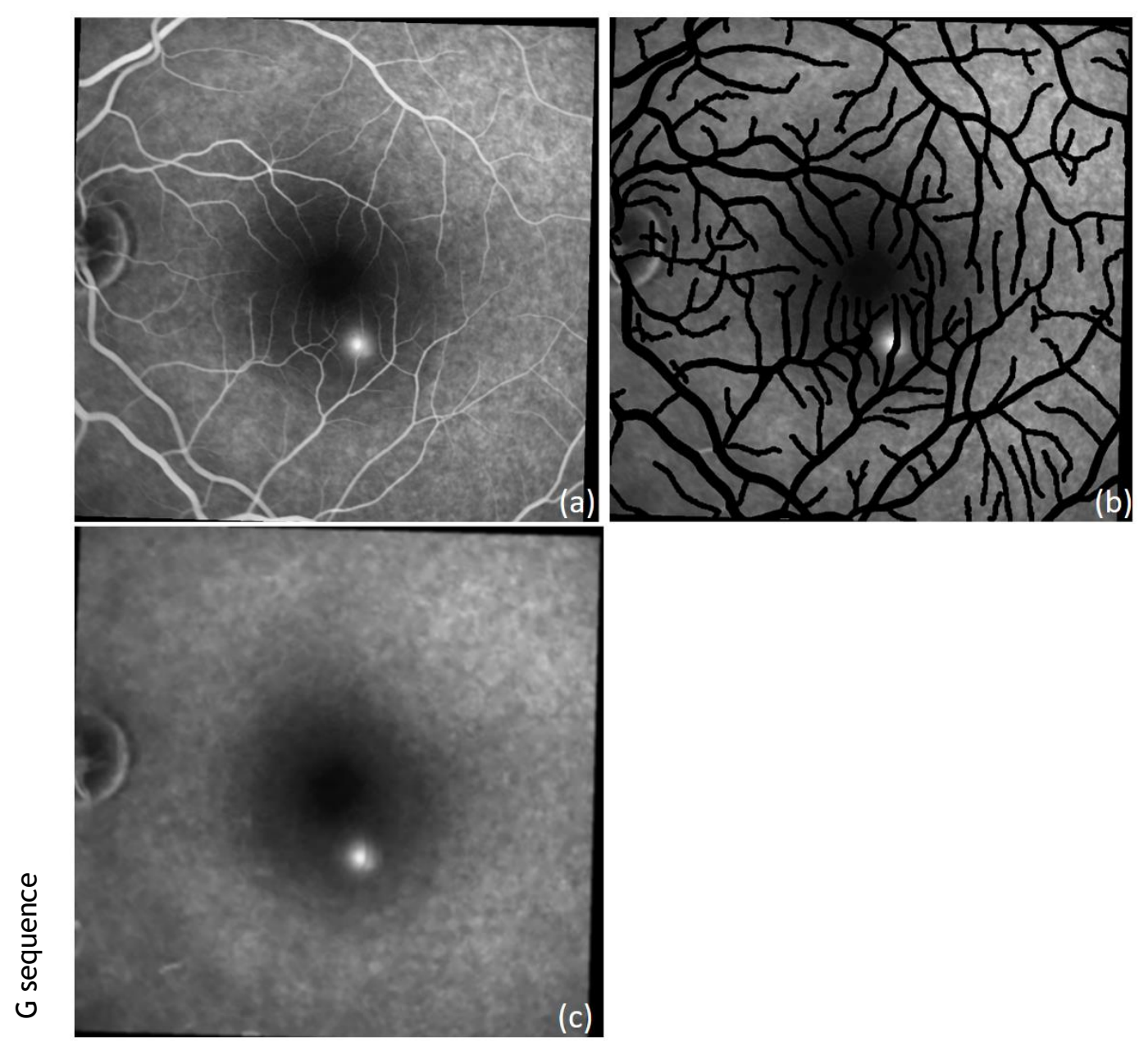

Figure 4.11 -Steps of vessel inpainting: input image (a), input image with vessels replaced by 0 (b) and image after vessel inpainting (c).

In cases where a leakage overlaps a region of the blood vessels, after inpainting, this region continues to have high intensity values because the neighboring pixels, leaks regions, are also regions with high intensity values. This is the only case where a vessel region maintains approximately the same intensity. In other cases, the regions of the blood vessels will have background values. 


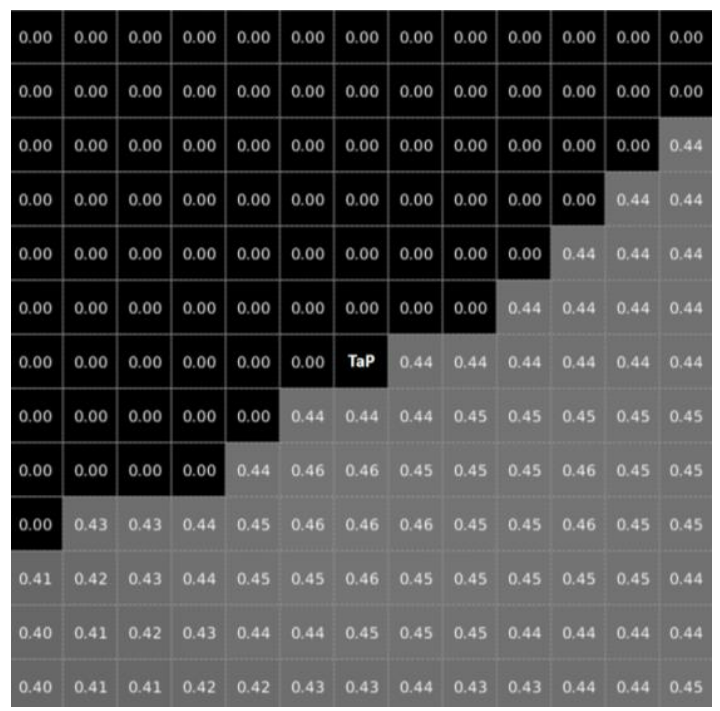

Figure 4.12 - Example of a window used in vessel inpainting.

\subsection{Optic Disc Detection}

The optic disc is the site of exit and entry of retinal vessels. In 2D images like the fluorescein angiography, it can be seen a region where there is large concentration of blood vessels. Furthermore, it appears as a dark region because it is a region without interaction with fluorescein. Since the images are centered on the macula, it is only possible to visualize half of the optic disc on a lateral border of the image. Under these conditions, it holds up to $1 / 6$ width of the image and a $3 / 10$ the height of the image. The optic disc search is performed in three steps. In the first step, it is considered that the optic disc is next to the image border that has the major concentration of blood vessels. In the second step, the optic disc is detected like a region in which the surrounding blood vessels have an inclination close to $0^{\circ}$. Finally, with the region determined in the previous step, the image row that has a smaller sum of the pixels is discovered. Since the optic disc is a dark circular structure, this line must correspond to the center of the optic disc and therefore, as the approximate size of the disc is known, the center detection allows the delimitation of the intended area.

In the first step, the optic disc search region is confined to the region with the largest number of white pixels along $1 / 6$ of the width of the image border. This allows to discover if the optic disc is on the right side (right eye) or the left side (left eye) of the image. For example, in figure 4.13, the next search steps were limited to the region near the right image border. This analysis is performed in the first image of the sequence, the only one where the blood vessels are segmented. 


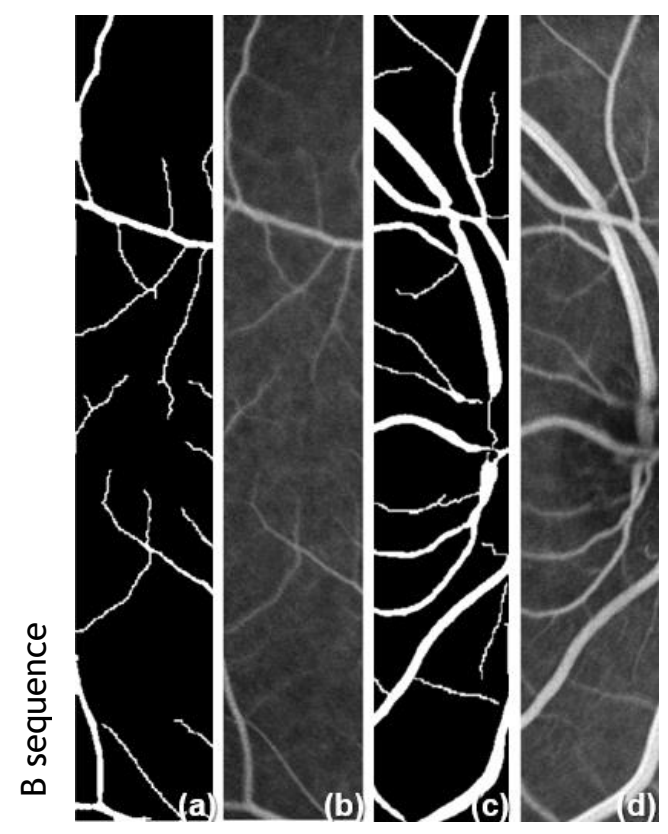

Figure 4.13 - Regions $1 / 6$ of the width of the image border compared in the first step of the optic disc detection algorithm: image with the segmented vessels on the left border (a), first frame on the left border (b), image with the segmented vessels on the right border (c) and first frame on the right border (d).

In the second step, a sliding window is passed through the previously determined region of analysis to search where the inclination of blood vessels is approximately $0^{\circ}$ which potentially corresponds to a location very close to the center of the optic disc (figure 4.14). The angle of inclination specifies the angle between the $x$-axis and the major axis of the ellipse that contains the region. The value ranges from -90 to 90 degrees. However, the achieved location is not accurate because the distribution of blood vessels above and below the optic disc is not uniformly balanced. The sliding window runs through the analysis region in a descendant direction. The size of the window is the same as the size expected for the optic disc. The angle of inclination calculated at each window is assigned to the center pixel window. The angle of inclination is a weighted average (equation 4.5), i.e., the result is the sum of the multiplication of the angles of inclination by the number of white pixels in each vessel, dividing at the end by the number of white pixels of the window.

$$
\theta_{i}=\frac{\sum_{j} \delta_{i, j} * n_{i, j}}{\sum_{j} n_{i, j}}
$$

where $\theta_{i}$ is the weighted mean angle of the vessels in sliding window $\mathrm{i}, \delta_{i, j}$ is the inclination of vessel $\mathrm{j}$ in the sliding window $\mathrm{i}$ and $n_{i, j}$ is the number of white pixels of vessel $\mathrm{j}$ in the sliding window i. In this way, in each window, the larger vessels have more relevance in this calculation.

The inclination for each vessel can only be calculated after the vessels are separated at the bifurcations. With a thinning algorithm, the centerlines of the blood vessels are obtained and 
the center of a bifurcation corresponds to a point where the connectivity is equal to or superior to 3. As the bifurcations are single points, these regions are dilated with a disk-shaped structuring element with a 10-pixel radius such these regions can be subtracted from the binary image with the blood vessels (figure 4.14). The thinning algorithm is composed by two subiteration. In the first one, the analyzed pixel is deleted if the four conditions are satisfied. First, its connectivity is one. Second, it has at least two black neighbors and not more than six around it. Third, at least one of the surrounding pixels on the right, above and left are white; and at least one of the surrounding pixels of the above, below and left are white. In the second sub-iteration, the first and second conditions are equal but the third and four change. This time, at least one of the surrounding pixels on the right, below and left need to be white; and at least one of the surrounding pixels of the above, below and right are white to be eliminated. If at the end of either sub-iteration there are no pixels to be deleted then the algorithm stops [59].

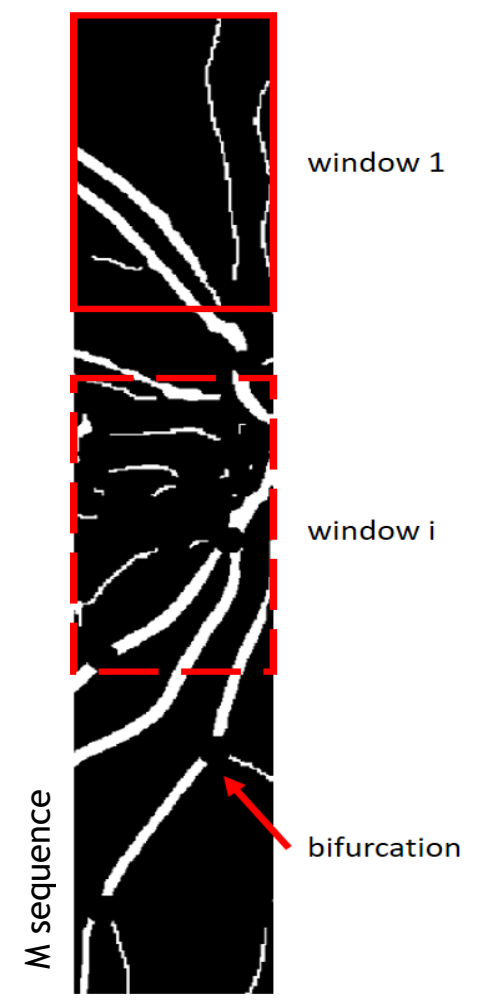

Figure 4.14 - Diagram representing the sliding window (representing $i$ the window number and $m$ an arbitrary value).

At the end of the second step, the optic disk search region becomes smaller. The search region for the third step corresponds to a region $3 / 20$ above and below the row in which the inclination of vessels is $0^{\circ}$. This region, which has the same height as the one expected by the optic disc, is expected to contain the actual center of the disc. For each sliding window corresponds a distance to the top of the image and an angle of inclination. Therefore, it is 
possible to establish a graph of the angles as function of the distance (figure 4.15). If there is more than one location where the graph passes through the $0^{\circ}$ angle, then one where the second derivative at the location is smaller is chosen. The variation of the angle as the sliding window descends in the region should be smooth, whereby the smaller second derivative is a good criterion.

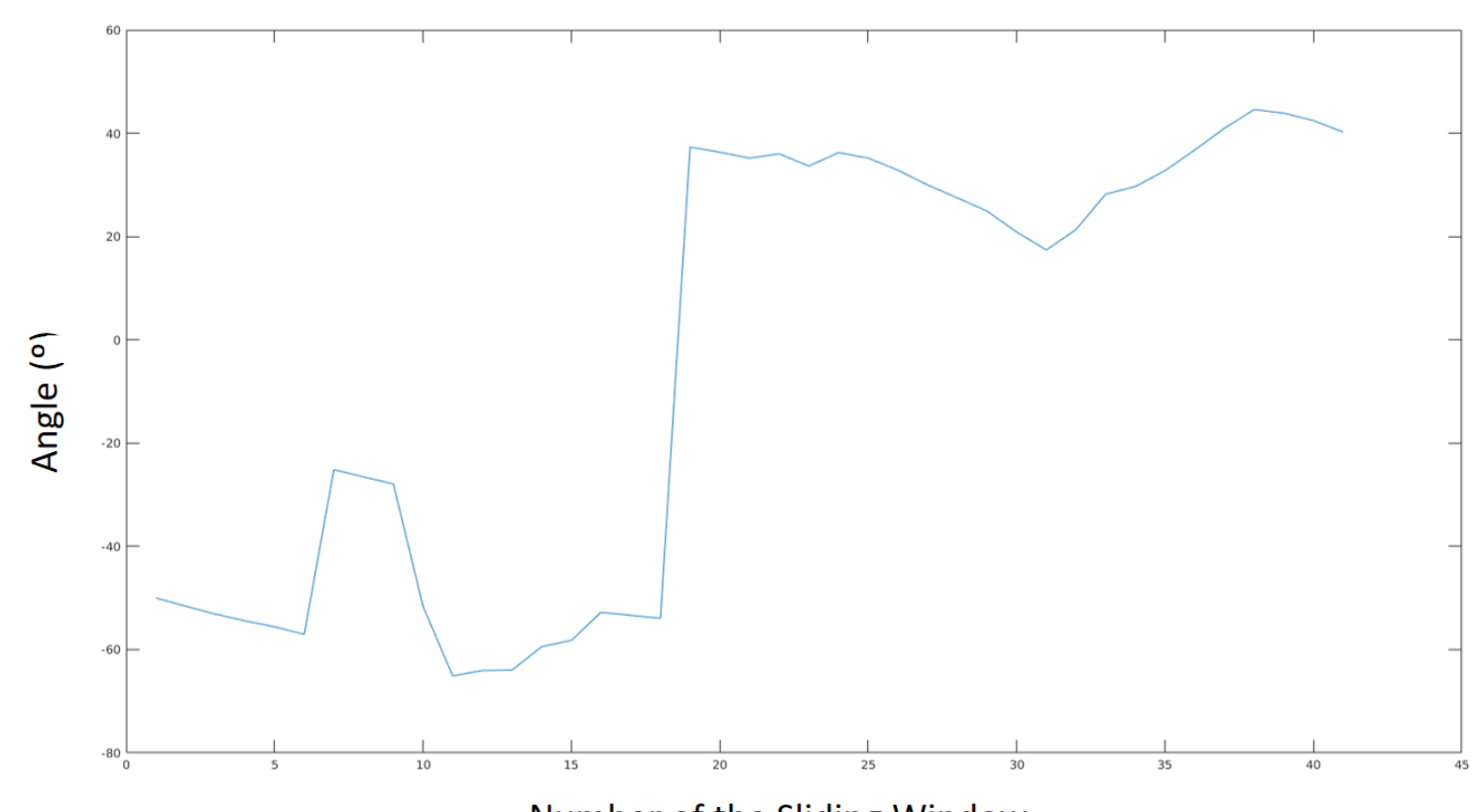

Figure 4.15 -Plot of the variation of the angle of inclination as the sliding window descends in the analysis region.

The process previously described is applied to the first image of the sequence after vessel inpainting. The region that results from the second step is searched for the row which the sum of pixels is smaller. This detection must match the center of the optic disc. As can be seen in the example of figure 4.16, the region in figure 4.16 (d) after this last step is better detected than after step 2 in figure 4.16 (c). The contrast of the figures 4.16 (b), (c) and (d) has been modified to allow better visualization. This step is not performed immediately after the first step because of the different luminosity that various locations of the image may have, the indicated location might not match the one desired. With limitation of the region through the second step, this last criterion allows a better detection. After the subtraction of images in a next step, the region considered to contain the optic disc is replaced by 0 .

The optic disc is not fully segmented because the leaks never appear in the detected region and to minimize the computing time of the overall algorithm. 

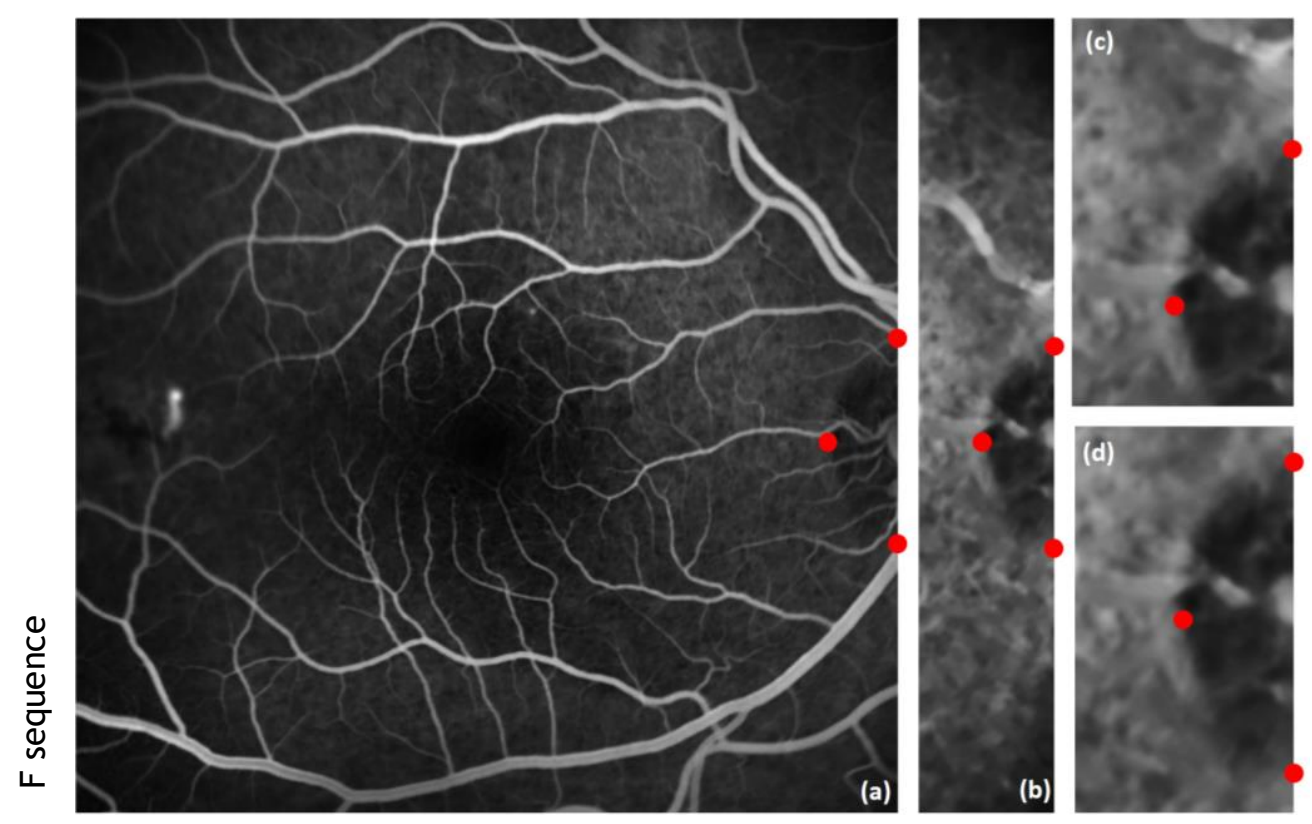

Figure 4.16 - Several steps of the algorithm with the top, the center and the bottom of the optic disc marked (red circle): first frame (a), region after step 1 (b), region after step 2 (c) and region after the last step (d).

\subsection{Intensity Normalization}

With the movement of the eye, the images do not fully correspond to the same region in the eye. After registration, the regions of the first image that do not match with the remaining images of the sequence are represented by pixels with 0 values. For the following steps of the algorithm, only the regions with correspondence are used. In the intensity normalization and background estimation, this fact is import since these regions cannot have influence in the process.

Due to luminosity, the intensity of the leaks and the background may be affected differently in the sequences. Background removal allows the background to have values close to 0 and the intensity of the lesions to be maintained or reduced little. However, if, for instance, the luminosity causes an overall decrease in the intensity of the images, the lesions continue to have low intensities and close to background values even after the background removal. In this way, a method of compensation of the intensity of the images needs to be introduced. The compensation method is intended to stablish the intensity of the background and allows the lesion intensity to become adequate.

Figure 4.17 shows two leakages (within the green and blue rectangles) at different times in the sequence. The luminosity diminished over time, and the red point, a leak point, has a lower intensity at a later stage. In the subtraction step from the early to the later frames, the region inside the green square would then be a low intensity region and could not be detected as a region of leakage. Despite this, the lesions within the blue square increased greatly in intensity. 
A second-degree polynomial is used to approximate the background of the images [60] and compensate the effects that modify the intensity of the retinal structures. To fit the seconddegree polynomial, 1000 well-distributed points are used throughout the image content. The first inpainted frame of the sequence serves as a reference for fitting the intensities of the remaining inpainted frames.
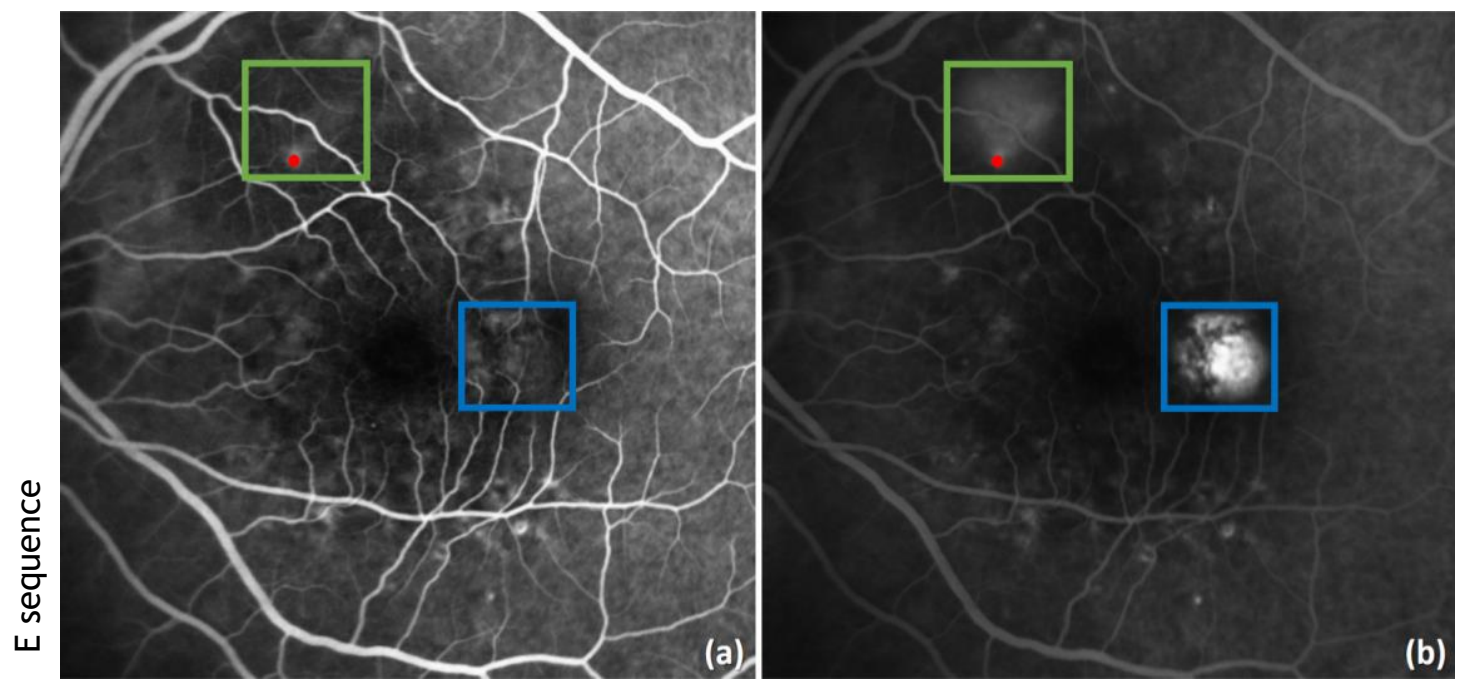

Figure 4.17 - Example of a situation where the luminosity affects the intensity of a leakage: early image (a) and late image (b).

The fit is achieved through an ordinary least squares (OLS) regression. This method minimizes the sum of squared errors (SSE) between the fit values and the selected values. In order to minimize the function SSE, the partial derivatives of the function with respect to each of the unknown coefficients of the fit function are calculated and equaled to zero. Then, the resulting system is solved. The equation 4.6 is the function implemented by SSE.

$$
\begin{gathered}
S S E=\sum_{i=1}^{n}\left(y_{i}-b_{0}-b_{1} x_{1 i}-b_{2} x_{2 i}-b_{3} x_{3 i}-b_{4} x_{1 i} x_{2 i}-b_{5} x_{1 i} x_{3 i}-b_{6} x_{2 i} x_{3 i}-b_{7} x_{2 i}^{2}\right. \\
\left.-b_{8} x_{3 i}^{2}\right)
\end{gathered}
$$

where $b_{j}$ for $j=0,1,3,4,5,6,7,8$ represents coefficients of the fit adjustment, $n$ is the number of selected points, $x_{1}$ are the intensities of the intended image for adjustment, $x_{2}, x_{3}$ are the coordinates of the image and $y$ are the intensities of the first frame.

Even with located border luminosities, the polynomial function can adjust the intensity, because they are regions in which gradually increases or decreases the intensity. On the other hand, leaks are regions that increase considerably in intensity relative to their neighborhoods, there is contrast, so that the polynomial function cannot be adapted, the intensity of lesions continues to be higher but may decrease little. The second-degree term of the intensity of the intended image to be adjusted is not used, precisely because the adjustment would be too 
much and could considerably change the intended intensities of the leaks. Figure 4.18 represents an example of the application of this step.
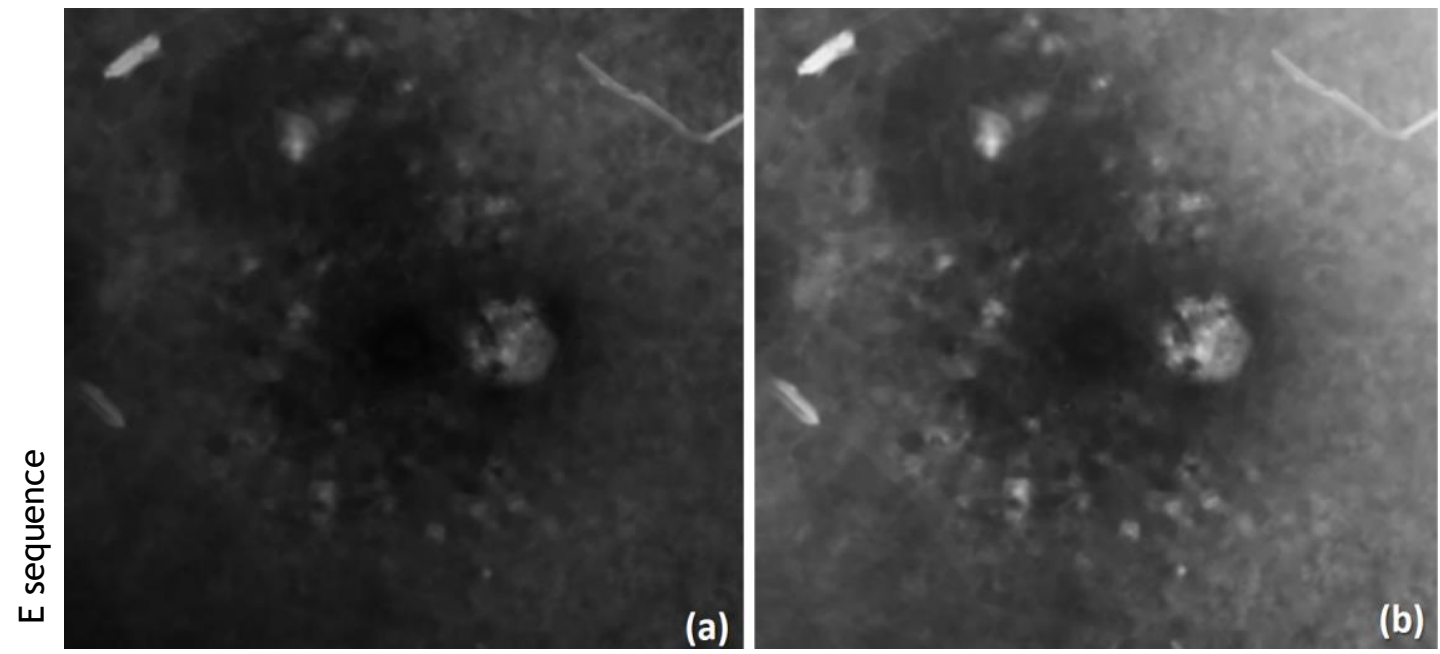

Figure 4.18 - Example of intensity normalization: before (a) and after (b).

\subsection{Background Removal}

The goal of background removal is also to reduce uneven luminosity of the image and this step is performed on the images after intensity normalization.

The background removal resorted to a morphological filtering. A morphological top-hat with a structuring element (square shape with 23 pixels width) is used to remove the background. The top-hat filter is an enhancement filter of bright objects on a dark background and it allows the background removal because it is the difference between the image and its opening (background estimation). As the opening is an eroding operation followed by a dilation operation, with this relatively large structing element, the dark regions (background) maintain approximately the same intensity of values but in the place of the bright regions (leaks) are less intensities, similar to the background. Thus, after subtraction, it is expected that lesion region exhibits a large contrast when compared to the remaining regions of the image. Figure 4.19 represents the different steps of the background removal algorithm. 


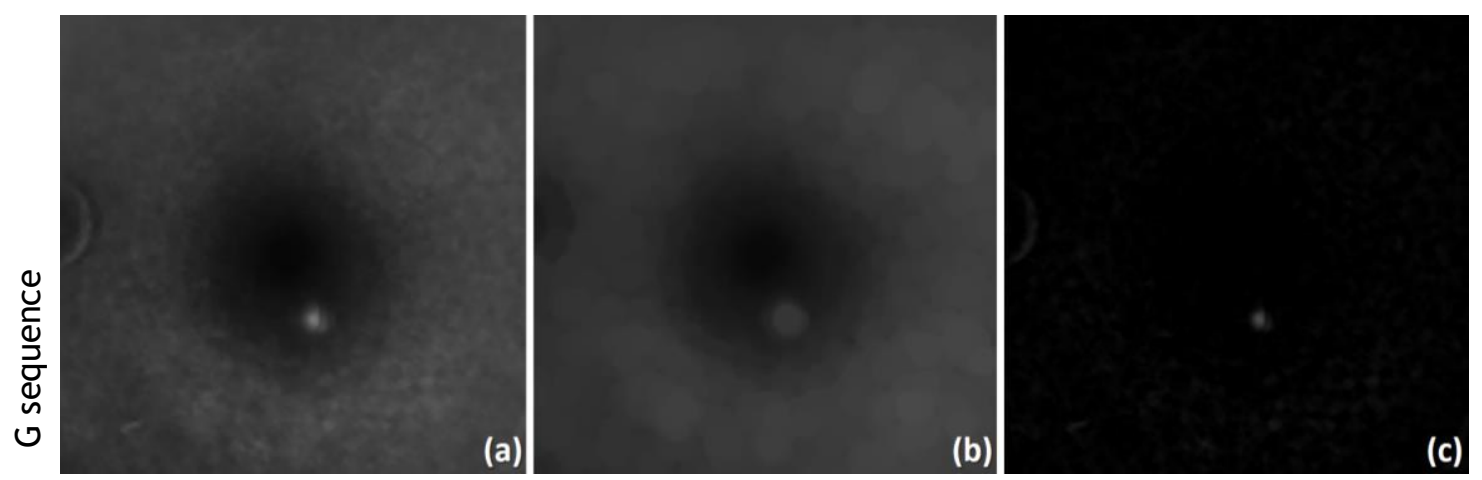

Figure 4.19 - Several steps of the background removal: image with inpainted vessel (a), background estimation with morphological opening (b), background removal (c).

\subsection{Image Subtraction}

Before image subtraction, the sequence of images needs to be divided manually into two parts: early phase and late phase. Image subtraction is performed between the mean of the late images and the mean of the early images of the sequence (figure 4.20). The used images are those in which the background and the optic were removed.

The lesion should not yet be visible or should be small in the early set. The late set of images can be larger to allow some problems that are eventually introduced by some algorithm step to be minimized.
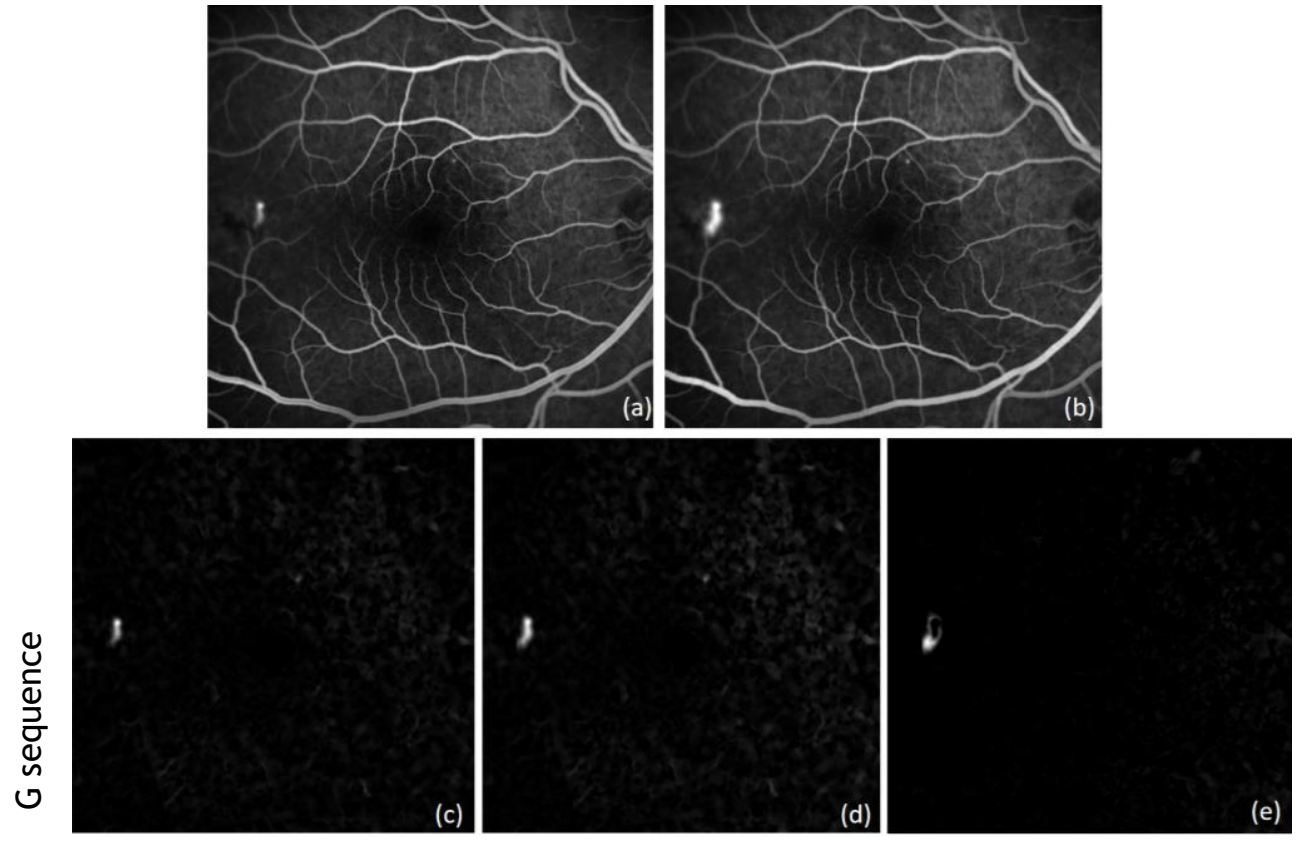

Figure 4.20 - Image subtraction steps: first image of the sequence (a), last image of the sequence (b), mean of the early frames after removal of background and optic disc (c), mean of the late frames after removal of background and optic disc (d) and subtraction between late and early processed frames (e). 


\subsection{Leakage Segmentation}

After several processing steps, an image is obtained in which the leaks correspond to the regions with high intensity. Therefore, the histogram of this type of images should be bimodal, with two predominant peaks. The first one corresponds to the background and artifacts resulting from the various processing steps, the second one corresponds to leakage regions. In this way, the Otsu method [61] can always be used for finding the threshold between these two classes. The Otsu method calculates the optimum threshold separating the two classes minimizing intraclass variance equivalent to maximizing inter-class variance.

In cases where the sequences do not present lesions, the histogram is not biomodal and therefore the Otsu method alone is insufficient. So, after the Otsu method, a white candidate region is excluded if the maximum intensity of the difference image without the backgrounds is 0.07 or the maximum difference in the difference image with the backgrounds is 0.1 , and the area is less than 150 pixels. A statistical analysis of the training data was conducted to find these parameters. Figure 4.21 demonstrates the steps of the Otsu method performed in this work.
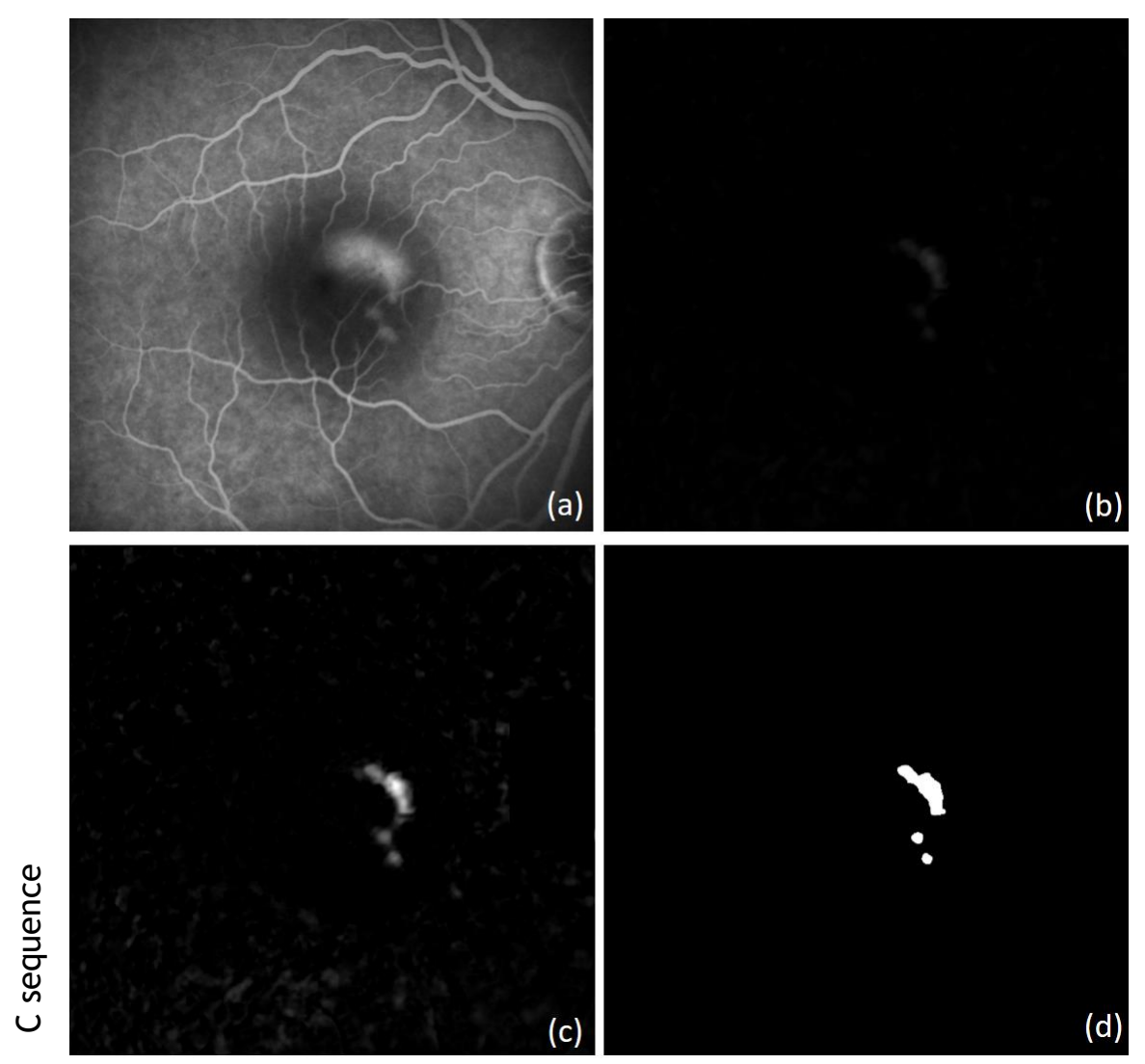

Figure 4.21 - Candidates identification with Otsu method: last frame of the sequence (a), subtraction between late and early processed frames (b), rescaled (0 to 1 ) image of the subtraction between late and early processed frames (c) and segmentation after Otsu method (d). 
The candidate regions after the Otsu method are regions that belong safely to leaks from the last image of the sequence. However, the regions do not correspond fully to the leaks in the last image of the sequence due to the processing undergone in the previous steps. Background removal and the mean of both sets (early and late images) contributed surely to a reduction of the size of the detected region. This region then needs to grow to achieve the correct leakage area. In order to determine this area, a region growing method is used (figure 4.22). The region growing algorithm is adequate when a seed point or a seed region are known and the initial region is somewhat smaller than the final segmentation. The growth is done using the last selected frame of the sequence.

The region growing algorithm iteratively examines neighboring pixels of the current segmented region. Neighbor pixels are added to the regions if they satisfy same conditions. In this region growing algorithm, these following are: (1) the initial region is the resulting region after the Otsu method; (2) the seed point is the point with the maximum intensity in the border of the initial region; (3) the growth in each iteration occurs based on equation 4.7 until no more pixels can be added.

$$
\begin{gathered}
I(x, y) \geq \min \left(R_{1}\right) \vee I(x, y) \geq \operatorname{mean}\left(R_{i}\right)-\left(\alpha *\left(\max \left(R_{1}\right)-\min \left(R_{1}\right)\right) \Rightarrow I(x, y) \in R_{i+1}\right. \\
I(x, y)<\min \left(R_{1}\right) \wedge I(x, y)<\operatorname{mean}\left(R_{i}\right)-\left(\alpha *\left(\max \left(R_{1}\right)-\min \left(R_{1}\right)\right) \Rightarrow R_{i+1}=R_{i}\right.
\end{gathered}
$$

where $I(x, y)$ is the visited pixel with coordinates $(x, y), R_{1}$ is the region after the Otsu method and $R_{i}$ is the region in iteration $\mathrm{i}$.

Since there is contrast between the lesion and the neighbor regions, all visited pixels that have at least the same intensity as the lowest intensity of the region after the Otsu method are included. The criteria should also be adapted to the growth, so in each iteration, the pixels are only added if they fulfill a conditions dependent on the average of the current segmented region. So, pixels are added that are equal to or greater than the difference between the region average and a threshold dependent on the initial region. In this work, the value selected for a was 0.35 . A cautious threshold was used so that the lesion area could not be exceeded. 

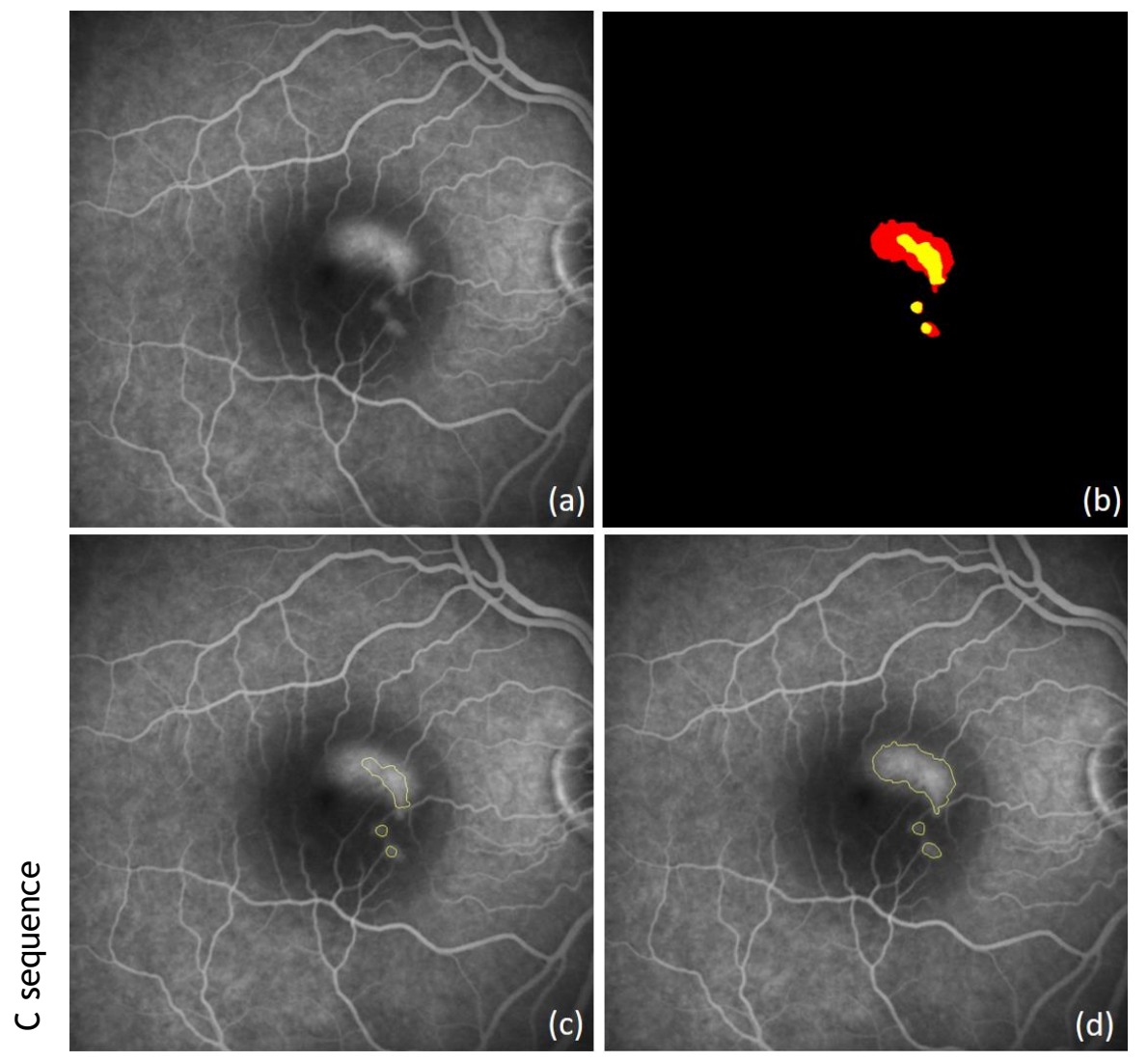

Figure 4.22 - Images of several steps until the region growing segmentation: late frame of the sequence (a), Segmentation of the lesion after the Otsu method (yellow) and segmentation of the lesion after the region growing method (yellow and red) (b), late frame of the sequence with the contour of the segmentation after the Otsu method (c) e after the region growing method $(d)$.

To obtain the growth of the lesions, it is necessary to obtain the segmentation of the remaining leaks of the angiographic sequence. Since it is expected that the lesion area does not vary greatly from frame to frame, an active contour algorithm is used. Each lesion contour is determined with the contour information of the frame immediately after. This segmentation is performed on images without blood vessels. The active contour method of Chan Vese et. al. [62] was used in this case. Like any active contour algorithm, this model allows the evolution of an initial curve in order to direct it to an image region that minimizes a cost function. Many active contour active methods are based on edge or gradient information to segment the desired boundary but Chan-Vese does not rely on gradient information, it depends on energy function. The model assumes that an image is approximated by two regions of constant and distinct intensities whose separation line is defined by the boundary (the separation curve) and the minimization of the energy term is guided by the equation 4.8 . 


$$
\begin{gathered}
F\left(c_{1}, c_{2}, C\right)=\mu \text {. Length }(C)+\vartheta \cdot \operatorname{Area}(\text { inside }(C))+\int_{\text {inside }(C)}\left|\mu_{0}(x, y)-c_{1}\right|^{2} d x d y \\
+\int_{\text {outside }(C)}\left|\mu_{0}(x, y)-c_{2}\right|^{2} d x d y
\end{gathered}
$$

where $\mathrm{C}$ is the curve, the input image is $\mu_{0}$, the smooth factor is $\mu$, the contraction bias is $\vartheta$, $F\left(c_{1}, c_{2}, C\right)$ is the function energy and the average of input image inside the curve $C$ and outside $C$ are respectively $c_{1}$ and $c_{2}$.

The smooth factor, contraction bias and contour initialization are the main factors that can be modified and improved to optimize the performance of equation 4.8. When the contraction bias is positive there is a tendency to contract and when it is negative there is tendency to expand. With a greater smooth factor, the perimeter of the region is smaller and the contour are smoother. In the algorithm, the chose smooth factor was 0.8 .

Two images with very close acquisition times may have different luminosity as already mentioned. This may lead to an image with a lower acquisition time to have an apparent larger leakage size than an image with a higher acquisition time. As the leak accumulates in the retinal space over time, the leakage region should increase gradually, it should be monotonous. This is known by comparing the mean intensity of the segmented region in the immediately following frame in the current frame, and in the immediately following inpainted vessel. Then, when the leakage region increases in intensity in the previous frame, the active contour algorithm is applied after the dilation of the segmented region of the frame immediately after. Otherwise, as there is a tendency to contract, important information about the lesion area would be possibly lost. Similarly, in the first contour determination of the last image of the sequence, after the region growing algorithm, the region is also dilated to determine a accurate segmentation. The radius size of the disk-shaped structuring element for the morphological dilation is dependent on the major axis of an ellipse containing the region. In the later image of the sequence, the radius of the structuring element used in the dilation of the region is 0.07 of the major axis of the ellipse containing the region. In all other cases, the radius is 0.03 of the major axis of the ellipse that contains the segmented region in the frame immediately after. Figure 4.23 (b) has a leakage size greater than figure 4.23 (a) although the luminosity induces an opposite idea. 

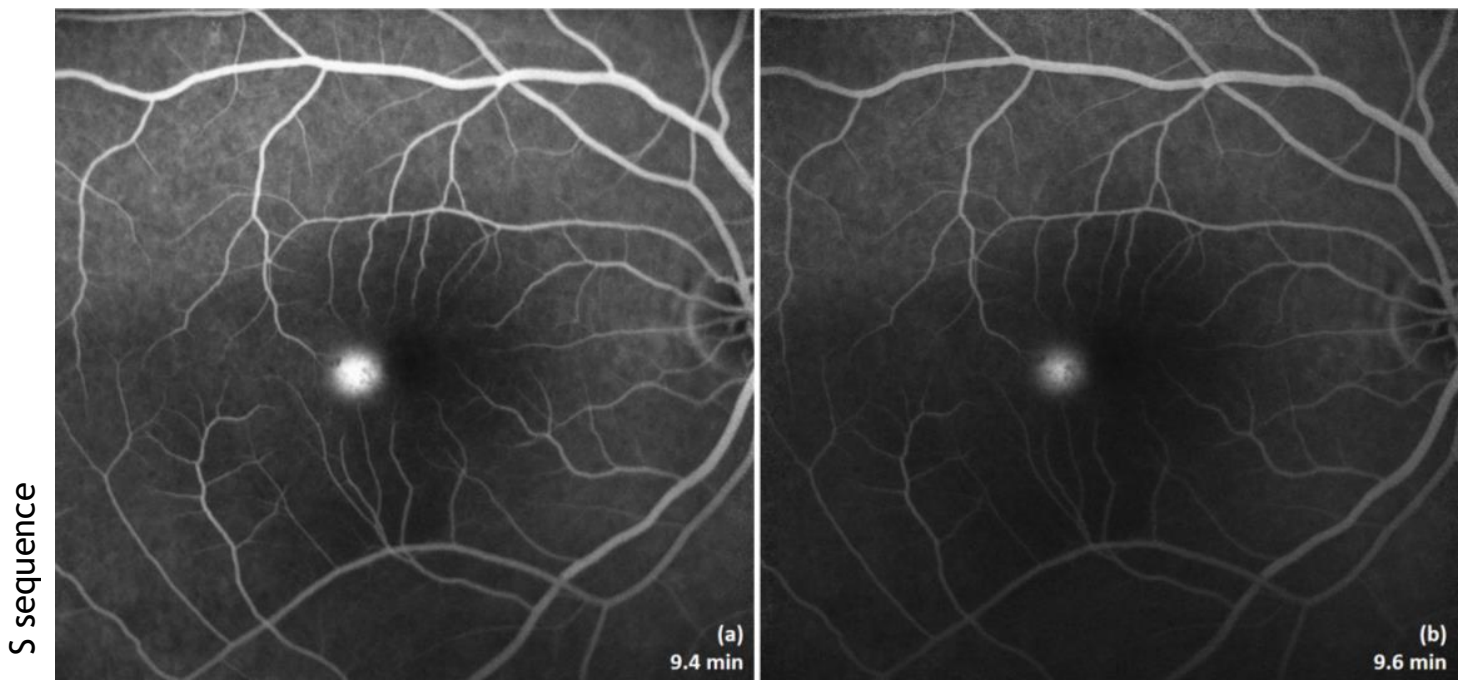

Figure 4.23 - Effect of luminosity on apparent leakage size at different times: 9.4 minutes (a) and 9.6 minutes (b).

Since the contour is as close as possible to the correct region to be segmented, it remains to find a suitable contraction bias. Between different consecutive images of the sequences, there may be very different lesion size variations. It is necessary to find an adaptive contraction bias to potentially correspond in any circumstances to variations in lesion size. If the image region of the inpainted vessels corresponding to the segmented region in the immediately after frame have a large pixel intensities dispersion, this may mean that a large contraction is required for the contour to conform to the desired shape. On the other hand, if the region has a fairly uniform pixel range, the contraction need not be too large to correspond to the limits of the leak. Therefore, the coefficient of variation was used to quantify the dispersion of pixel intensities. In fact, the coefficient of variation is a measure of spread that describes the amount of variability relative to the mean (equation 4.9 ).

$$
C V=\frac{\sigma}{\mu}
$$

where $C V$ is the coefficient of variation, $\sigma$ is the standard deviation of the segmented region of the immediately later frame and $\mu$ is the mean of the segmented region of the frame immediately later.

The same standard deviation can be considered large or small, depending on the magnitude of the mean, so the coefficient of variation is adequate in this case to compare the dispersion of the intensities of the pixels in different images. The contraction bias for each case can thus be calculated as dependent on the coefficient of variation. However, in the case where the lesion increases considerably in size throughout the sequence (and it does not appear in the early frames) a minimum contraction bias is guaranteed. These regions do not have shared points with those detected with the sliding band filter. A minimum contraction bias of 0.5 is used, if the frame contour is of the dilated segmented region of the frame immediately later, 
and 0.45 if only the contour of the segmented region of the following frame is used. In the case where the input contour of the active contour algorithm results from the dilated segmented region of the nearly later frame, the induced contraction is greater, since the dilation may lead to some distance from the region considered adequate. Figure 4.24 briefly schematizes the choice of the contraction bias.

It is expected that the time difference between frames is very close and therefore the contour is also close and the correct segmentation can be achieved. However, in very large differences between frames and therefore in the lesion size, the active contour algorithm has difficulty adapting to the desired shape. In these cases, where the difference between frames is greater than 5 minutes, the region growing algorithm is applied. The growing region is guided by equation 4.7 , where the seed region only includes the point of greatest intensity of the immediately later segmented region in the current inpainted vessel image (with $a=0.2$ ). These cases are rare or even non-existent.

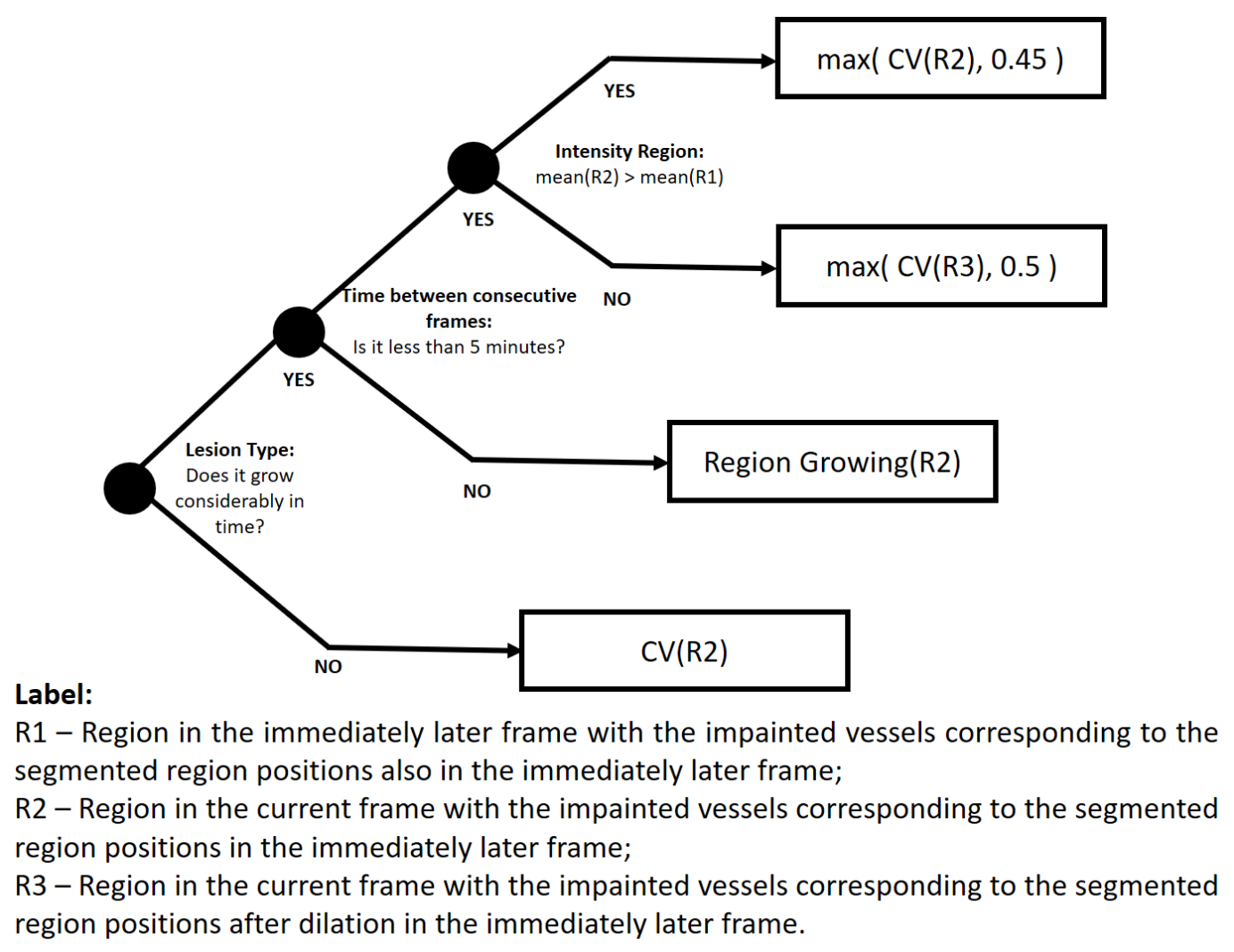

Figure 4.24 - Decision tree for the contraction bias.

The active contour algorithm allows the segmentation of the contours of the lesion in each image sequence. There is a moment when the leak still is not visible even though the algorithm can continually segment regions that do not correspond to anything. In order to turn this situation, the difference between the neighborhoods of the leak and the leak itself is evaluated. The detected region is dilated with a disk-shaped structuring element with a radius of 20 pixels. 
This new additional region that arises after the dilation is considered as the surrounding region. Though a statistical analysis with the training data, it was verified that when the difference between the region considered as leakage and the surrounding region is less than 0.03 , then the region should not be considered as having an lesion.

Figure 4.25 shows all the leakage segmentation steps of the algorithm.
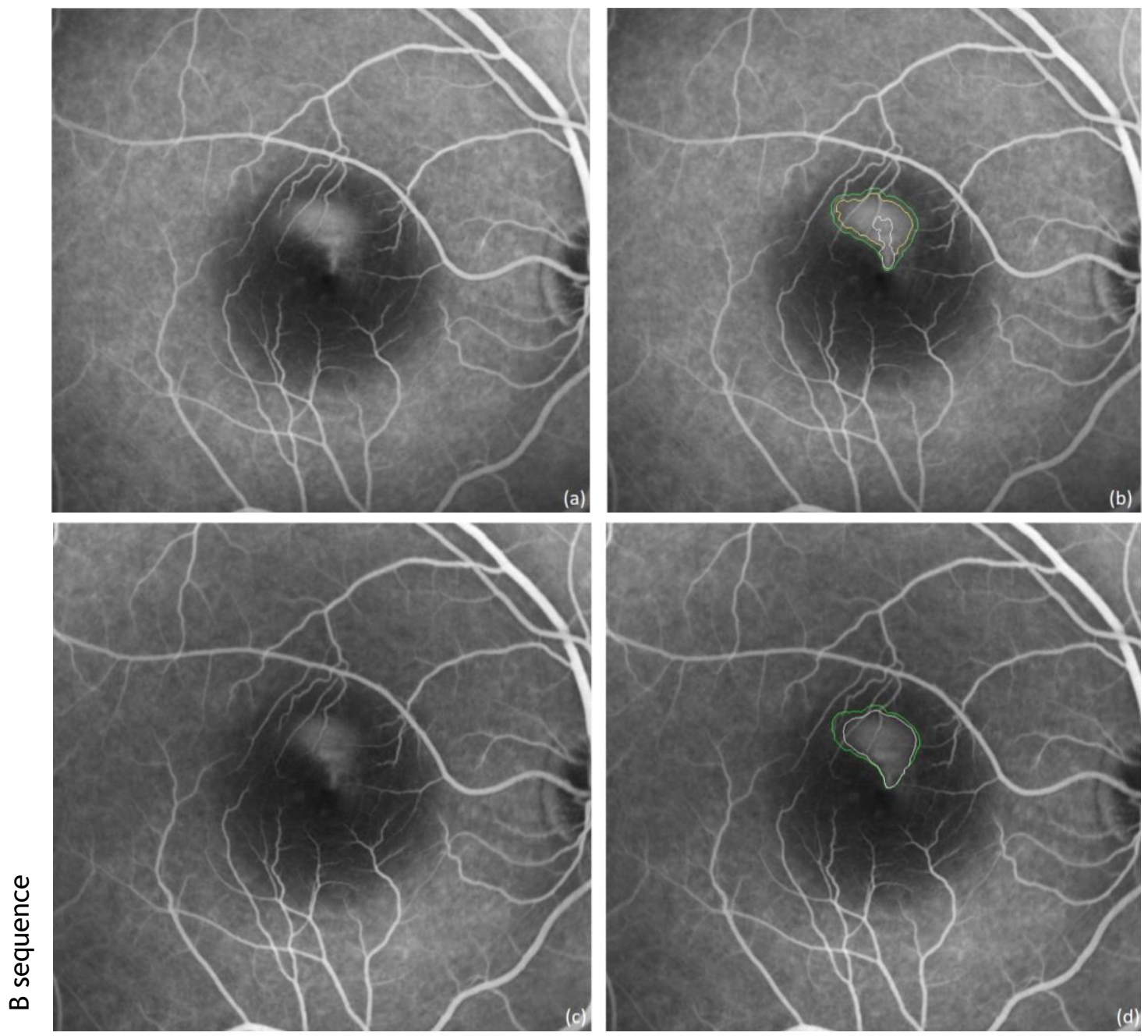

Figure 4.25 - Contour progression of the lesions in the segmentation algorithm: last frame of the sequence without contour (a) and with contour (white - contour after Otsu method, yellow - contour after region growing, green - contour after active contour algorithm) (b); second-last image of the sequence without contour (c) and with contour (green - contour of the frame immediately later, white - contour after active contour algorithm).

To ensure correct final segmentation, only the monotony of regions remains to be guaranteed. The decision if each pixel belongs to the segmented region is done by analyzing 5 images ( 2 immediately preceding frames, 2 immediately after frames and the current frame). A pixel belongs to the final segmentation region if it fulfills the criteria indicated in table 4.3. For instance, if the pixel belongs to the immediately preceding and immediately following 
frames, then it belongs to the segmentation region in the current image even if it were not after the active contour algorithm. With this approach pixels that appear only punctually in the segmentation region are not included.

The last condition of the table 4.3 is not very common, but situations were identified in training in which the algorithm had problems of detecting regions that were part of the real leakage in later frames, but the active contours could recover these regions in earlier frames. Note that the active contour algorithm is applied from the latest frame to the earliest frame. Since monotony is a characteristic of this type of leakages, if a set of pixels appears systematically in the earliest frames, it will also be surely part of the lesion area of the later frames.

Table 4.3 - Conditions to guarantee monotony of leaks growth.

\begin{tabular}{|c|c|c|c|c|c|c|}
\hline \multicolumn{5}{|c|}{ Index of the image (i) } & \multirow{3}{*}{$\begin{array}{l}\text { Decision to belong } \\
\text { to the leakage }(\mathrm{R}) \\
\text { to ensure monotony }\end{array}$} & \multirow{3}{*}{ Observations } \\
\hline$i-2$ & $\mathrm{i}-1$ & $\mathrm{i}$ & $\mathrm{i}+1$ & $i+2$ & & \\
\hline \multicolumn{5}{|c|}{ Class of the pixel I $(x, y)$} & & \\
\hline 0 or $\mathrm{X}$ & $x$ & 0 & $x$ & $\mathrm{X}$ & $I(x, y) \in R_{n, i}$ & $\begin{array}{l}\text { Situation in which there was a problem and } \\
\text { the pixel does belong to the segmented } \\
\text { region although it belongs to the surrounding } \\
\text { images }\end{array}$ \\
\hline 0 & 0 & $x$ & $x$ & $\mathrm{X}$ & $I(x, y) \in R_{n, i}$ & $\begin{array}{l}\text { Normal situation, the pixel begins to belong } \\
\text { to the region in a certain moment of time of } \\
\text { the sequence }\end{array}$ \\
\hline$x$ & $\mathrm{x}$ & 0 & 0 & 0 & $\begin{array}{l}I(x, y) \in R_{n, i} \\
I(x, y) \in R_{n, i+1} \\
I(x, y) \in R_{n, i+2}\end{array}$ & $\begin{array}{l}\text { Situation in which it is discovered that as a } \\
\text { pixel is included in more early moments in } \\
\text { the sequence, then also to be included in } \\
\text { later moments to have monotony }\end{array}$ \\
\hline
\end{tabular}

Label:

$X$ and 0 respectively represent a pixel belonging to and not belonging to a region of leakage after the active contour algorithm; $\mathrm{I}(\mathrm{x}, \mathrm{y})$ represents a pixel of the image with $(\mathrm{x}, \mathrm{y})$ coordinates; $R_{n, i}$ represents a leak region $n$ in a frame $i$ of the sequence. 


\section{Chapter 5}

\section{Results and Discussion}

\subsection{Pros and Cons of the Algorithm Steps}

Since leak detection has so many challenges, the algorithm consists of many steps. As the test set was small, machine learning techniques were not used for the detection of the lesion. Each step should be analyzed individually to understand potential problems in the final segmentation. Figure 5.1 shows all the steps of the algorithm until reaching the final segmentation. The sequence represented has 19 images but the operation is demonstrated in only 3 of them (the first selected frame, an intermediate frame and last one). In the leakage segmentation steps, with the region growing and with the active contours, the region contours are marked red.

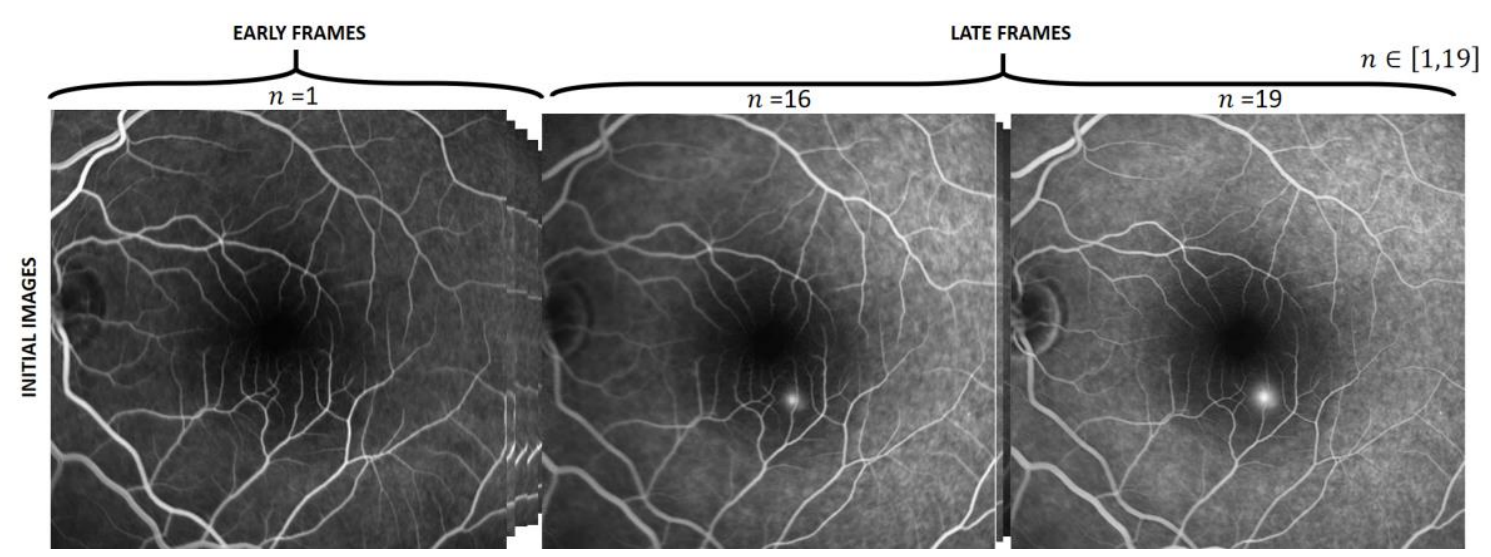




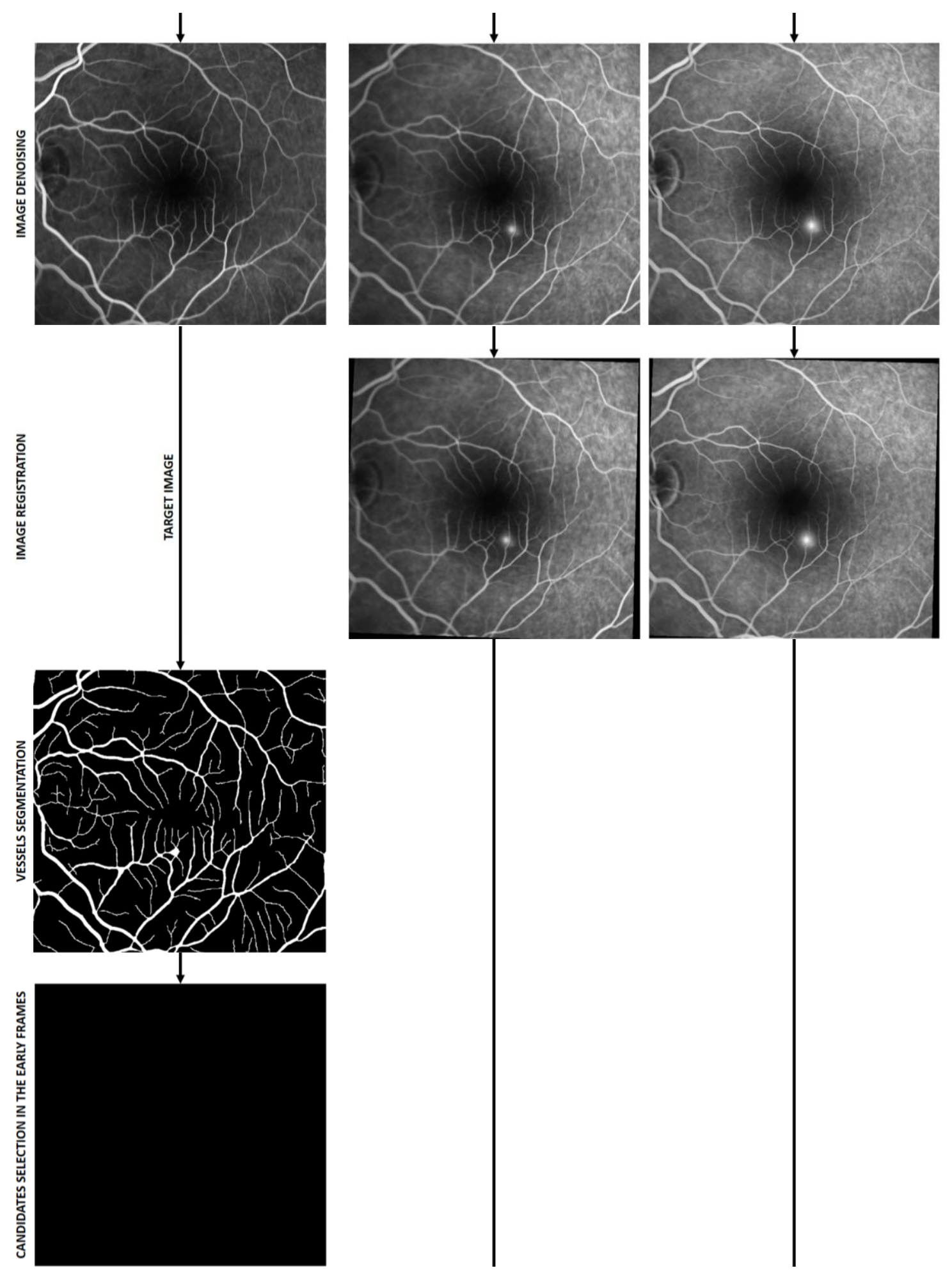




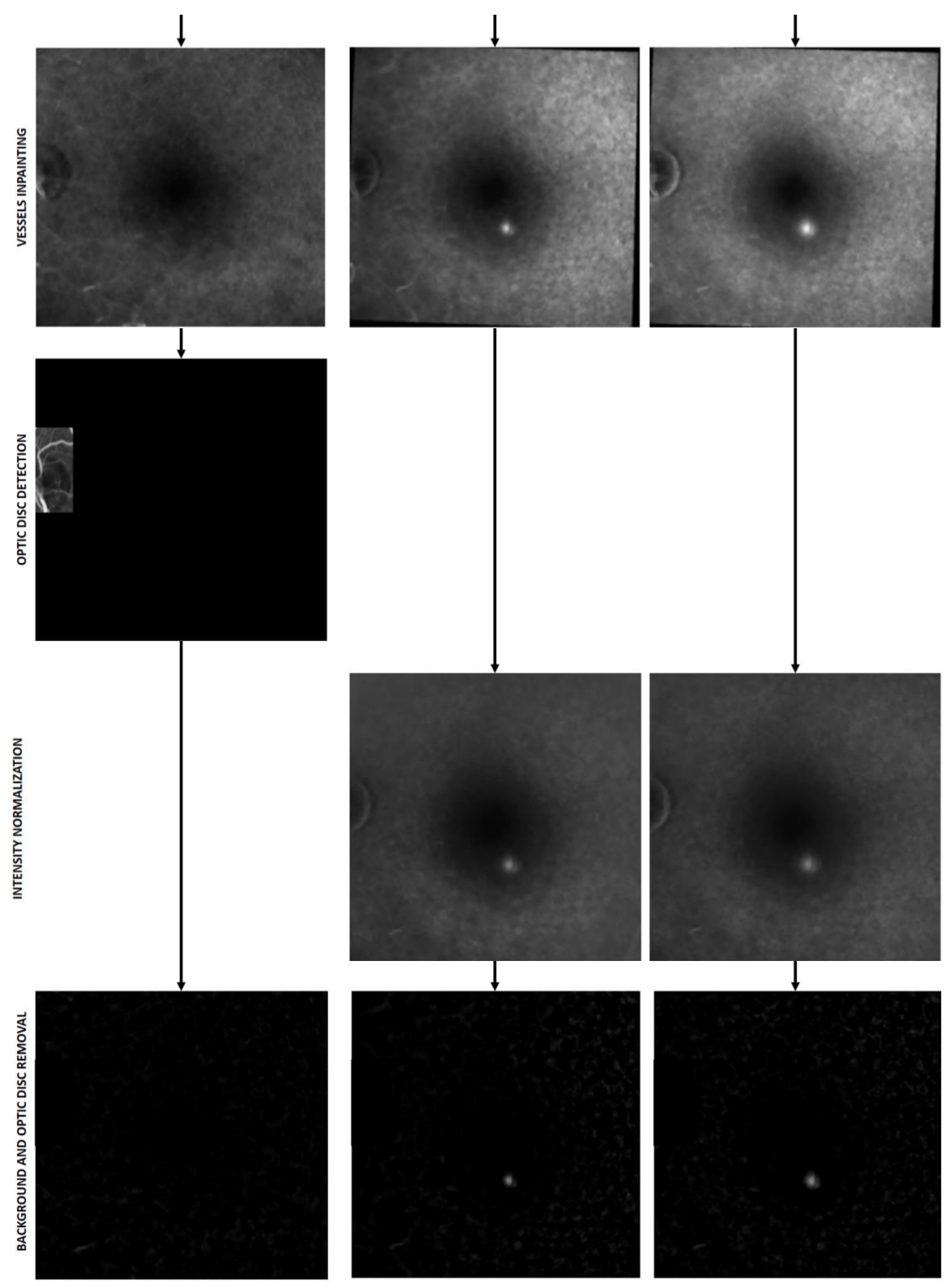




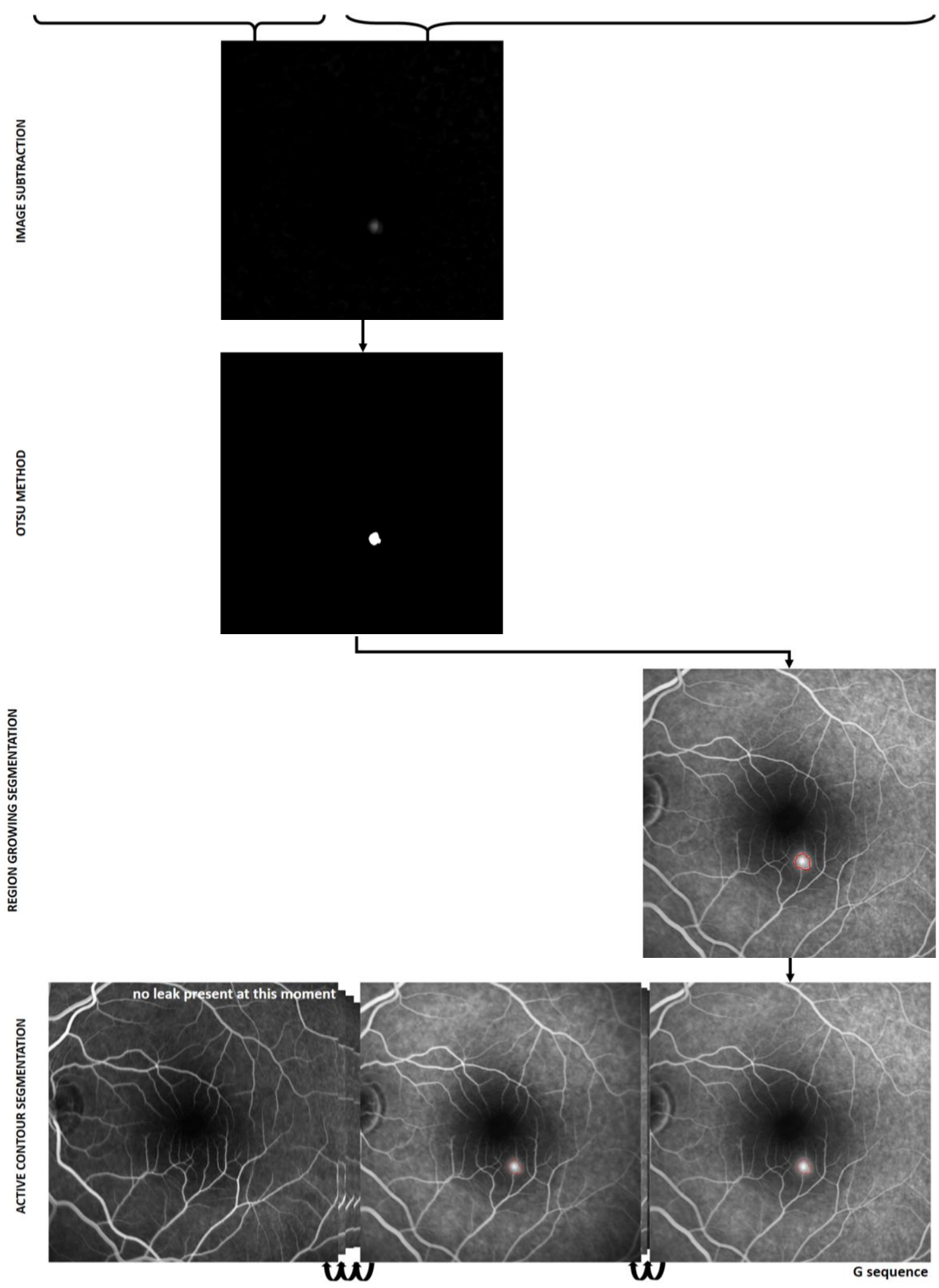

Figure 5.1 - Intermediate and final results of the developed algorithm.

The manual selection of frame should only be used for selecting the appropriate images of the angiographic and to separate the sequence correctly in early frames (no lesion or little presence) and late frames. 
The time detection algorithm works on $100 \%$ of the sequence. Indeed, it is quite robust in character recognition. It even works for different image dimensions. On the other hand, it is dependent on the position in the footer and only works for a $30^{\circ}$ field of view. A possible future solution would be automatically determining the footer region and examine all content through character recognition.

The processing begins at the denoising stages. The size of the filters is rather small but seems to solve the noise problems encountered at the start. A small filter also induces a smaller change in the intensity of the pixels which is an advantage in the case of lesions and vessels because the contours remain practically unchanged.

Registration is an essential step so improper registration can affect the leakage detection result. The registration has only been visually evaluated. In overall, the registration is efficient, although minor distortion in some vessels were verified. The main reasons for these minor distortions are the poor image quality and strong differences in image luminosity. The poor quality of an image can result in the illusion of elastic behaviour. This situation would not be expected since movements of eyes are almost uniform. As can be seen on the red rectangle of figure 5.2, there is a discontinuity of the vessel. These phenomena may be due to sudden movements of the patient's eye, inducing characteristics of images different from those expected. In figure 5.3, an example is shown in which there was a little distortion (represented by red rectangles) in the registration process.

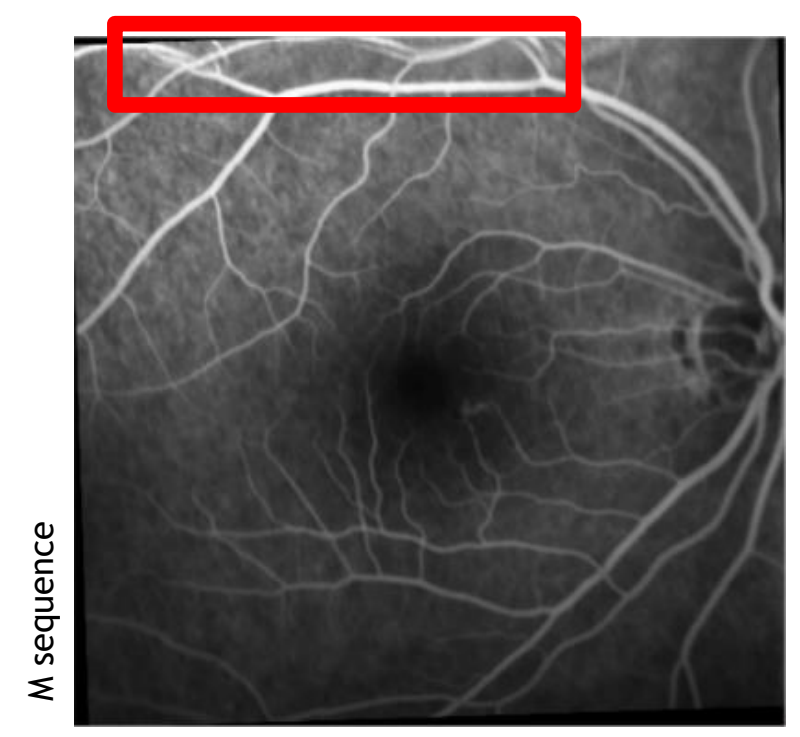

Figure 5.2 - FA image with bad acquisition quality. 


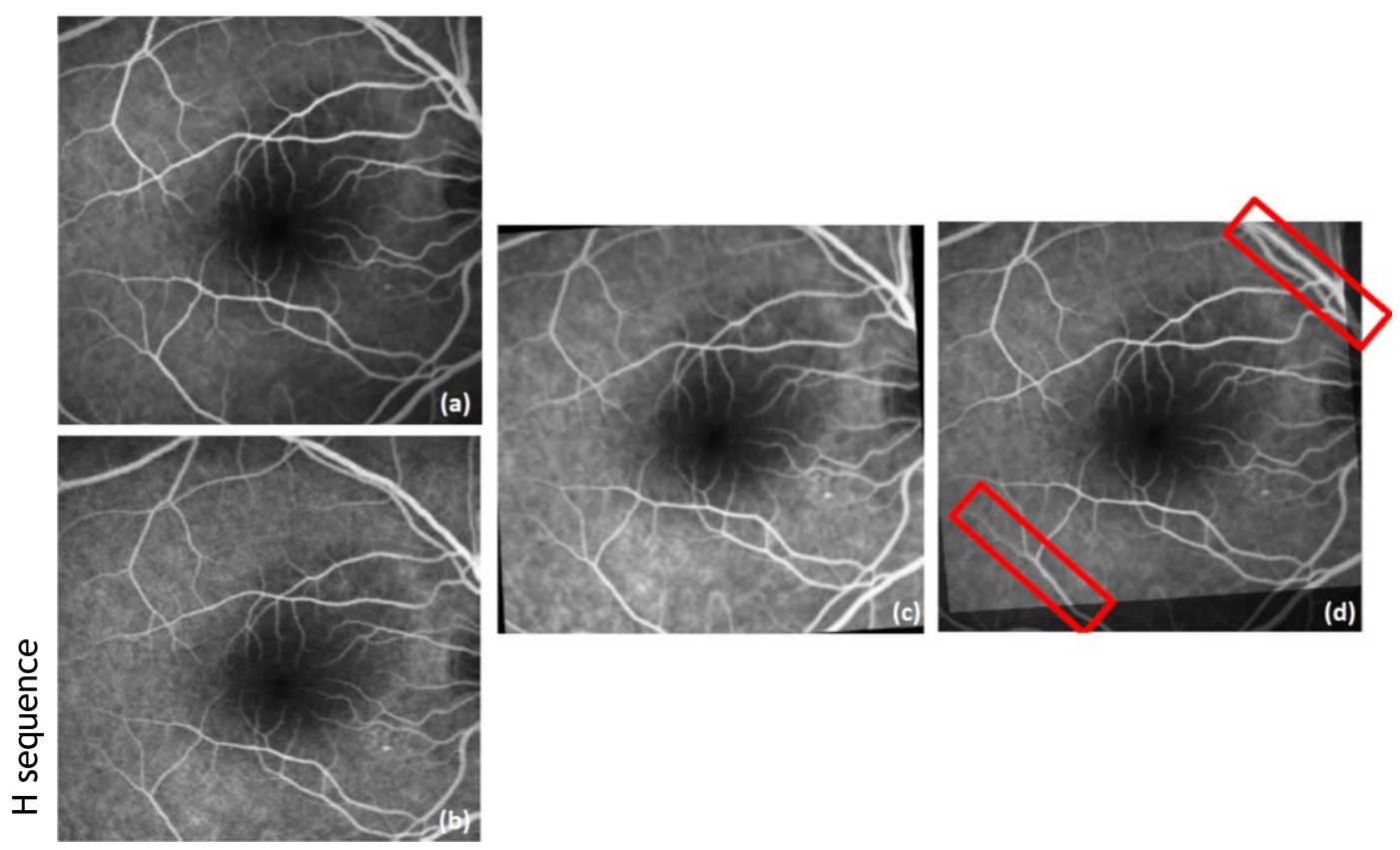

Figure 5.3 - Example of a registration that had minor distortions: target image (a), source image (b), registered image (c) and mean between the target and registered images (d).

SURF proved to be, in fact, a good methodology for detecting blobs (it catches vessel bifurcations) and it is invariant to rotation and scaling. With the optimization of parameters through pyramid techniques, many points were detected. The metric threshold, the match threshold and the maximum ratio were respectively 60,15 and 0.65 . As many points have been detected with matching and pair elimination, the required number of points to perform image registration is achieved in all images. The matching points are not usually in leakage region, as intended, because a lesion is a region with quite distinct features in the different frames.

The main problem of the blood vessel segmentation is that it normally includes leakage regions in the images that appeared in the first image of the sequence. A step was introduced in the algorithm to avoid this problem. Therefore, the main cons of this method for segmenting vessels are the nearby vessels appear connected and in areas where there is poor luminosity the vessels may not fill completely (figure 5.4). If a lesion appears at a later stage in the place where the vessels appears connected, then it would be impossible to consider this as a leaked region since in the images with the inpainting vessels it should correspond to a region with the same pixel tonality of the background. It would be a case of a false negative. In case the vessels are not fully filled and considerably increase in intensity throughout the sequence, it may turn this as a leaked region, so that there would be a case of a false positive. Despite this, the algorithm visually presents an excellent detection mainly in complicated cases of small blood vessels. 

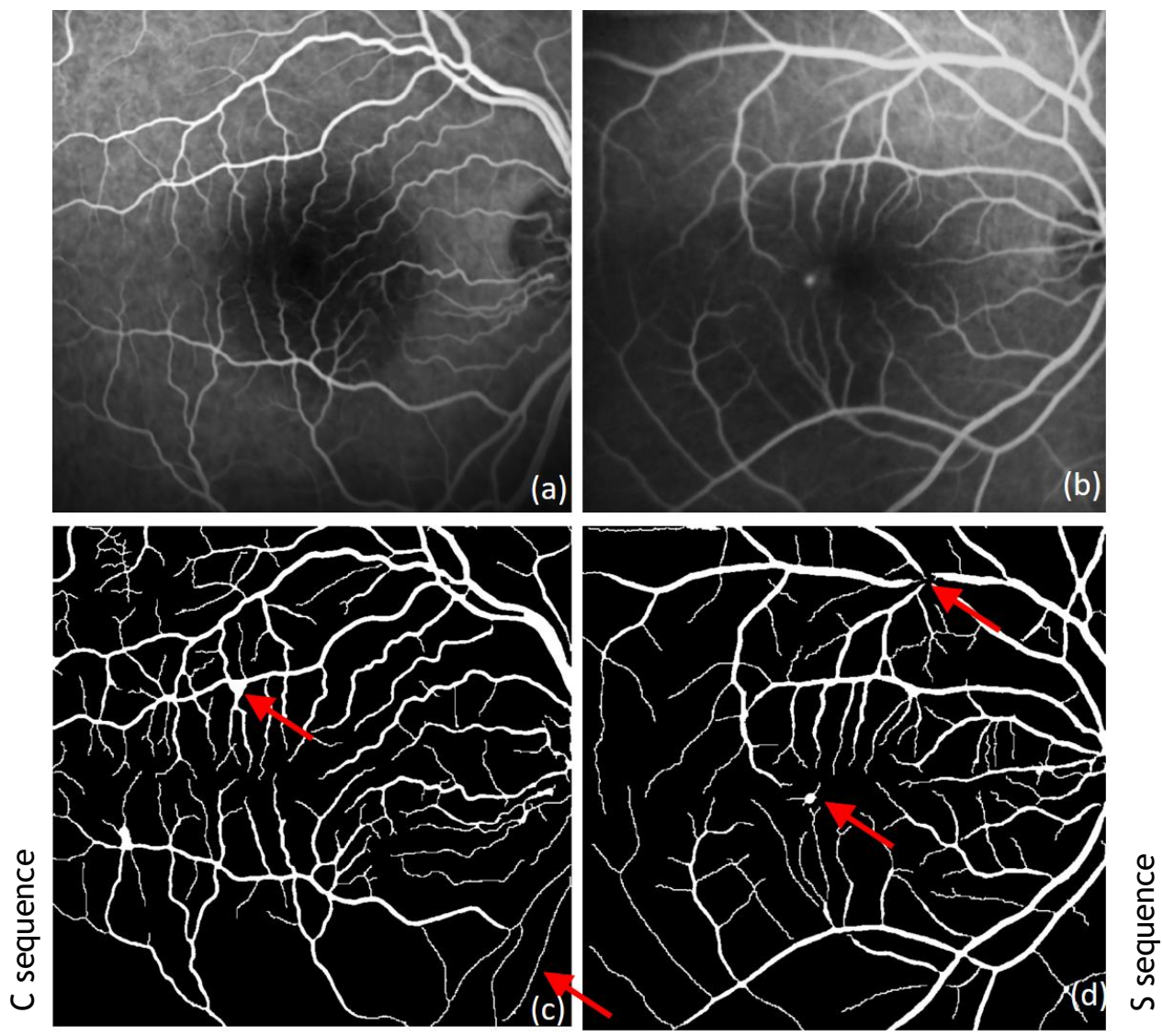

Figure 5.4 - Example of a vessel segmentation that had some problems: original image ( $a$ and b), vessel tree with connected vessels and filling problems (c) and vessel tree with leakage detection and filling problems (d).

The search for potential leakage regions in the first image of the sequence is performed because some types of lesions have early manifestations. The cons of this step are: the small leaks may not be detected and not leakage regions may be considered as potential lesions candidates. In the first case, if the vessel segmentation algorithm includes a leak region that is not detected by this step, there will be an incorrect delimitation of the contours in the early sequence images. In the second case, detection of non-lesion areas, typically vessel regions, may not be a problem if the regions do not increase in intensity throughout the sequence. In this way, the region will not be a candidate for lesion after frame subtraction. In figure 5.5 a blob is detected and it corresponds to a region of vessels bifurcation (yellow circle). 


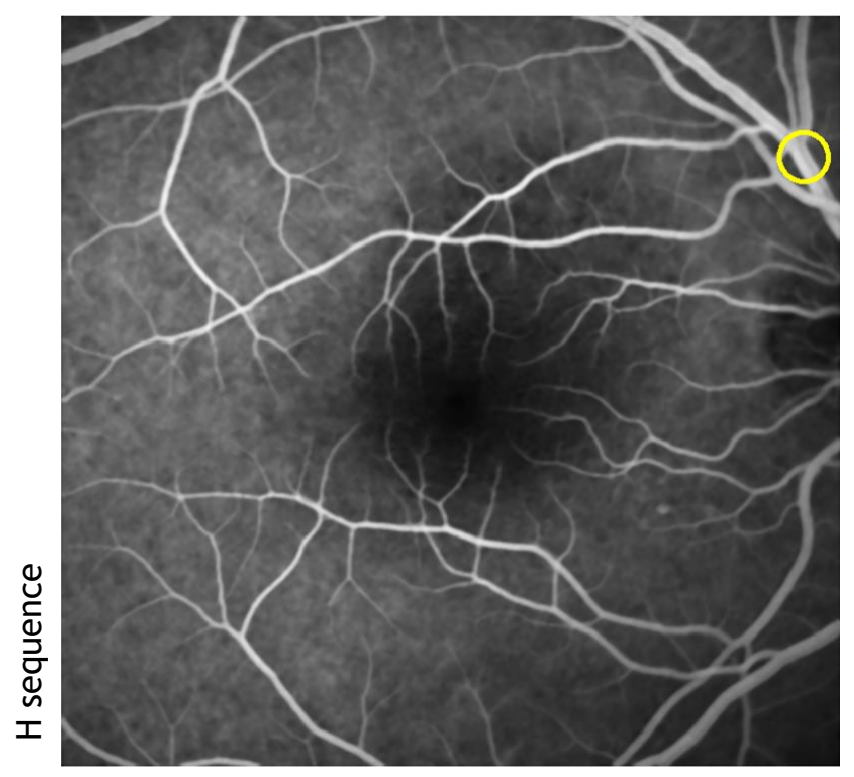

Figure 5.5 - Example of detecting a region that does not correspond to leakage (yellow circle).

In vessel inpainting the mask of the detected vessels is dilated. This consideration can sometimes be a pro or a con of the algorithm. It is a pro when the dilation allows to dissipate the small difference in the vessel registration. It is a con when the dilation next to the leakage region distort the real contours. A disk-shaped structuring element having a diameter slightly less than a quarter of the width of the vessels was used with the aim of accomplishing a balance between the potential pros and cons of dilating the vessel mask. Despite all, the method seems to be quite effective in replacing the vessels.

Optic disc detection is quite effective. The main problem of the method is, however, that it is dependent on the size and position of the optic disc. Further, as the first detection is based on the maximum number of white pixels attached to the borders, if there is a lesion near to the border of the image in the first image of the sequence, this consideration may no longer be valid. The detection of the correct area of the optic disc is only compromised in step 3, if the vessels are not well filled and therefore the inpainting of the vessels also fails to cover all the effective vessel region.

Due to luminosity phenomena, the mean intensity of the leakages may decrease over time, which was not expected. Then, as in these cases, the leakages have low intensities, the background removal and the frame subtraction made it difficult to detect these region as candidates for leakage. An intensity normalization method has applied. However, it can have some disadvantages like the reduction of the intensity of the lesions in some frames, and the blurred aspect when the adjustment cannot be completely adapted. In the first case, the background and the leakage get low contrast, but the background becomes more uniform, which is more advantageous in its removal and when subtracting. In the second case, despite 
the blurred, the intensity values are close to the background. An example of what happened is shown in figure 5.6. The red line delimits the blurred region.

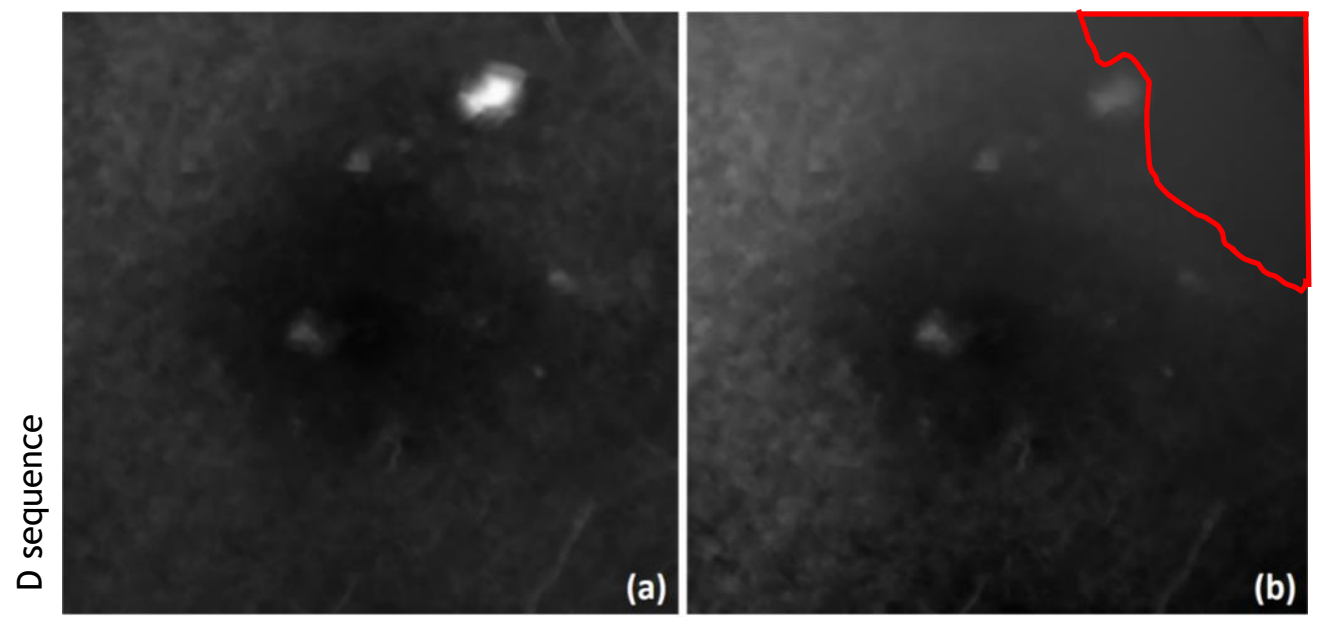

Figure 5.6 - Example of a situation where there is a region that is blurred and the leakage intensities decrease a little: vessel inpainting image (a), output image (b).

In the case of background removal, lesions with poor contrast to the surrounding area may disappear. As the normalization of intensity already provides a good fit with the background, only the top-hat filter is applied with a small radius disk-shaped structural element to remove any possible mismatches in the background intensities.

The subtraction of late frames by early frames is the most essential step. The previous steps ensure that the influence of regions similar to vessels is minimized in the final leakage segmentation, but this step allows to locate lesion regions to grow along the sequence because they increase in intensity and size during examination. A careful choice of images should be made so that there is a difference in the intensity of the lesions.

The Otsu method may fail when the lesions are very small, with no considerable peak in the histogram of the images and in case there are leakages of different intensity means, in which several peaks are expressed. With the step of intensity normalization, the mean intensity of different leakages seems to be closer as it is possible to prove in figure 5.6. There are also minimum criteria for regions to be considered as candidates. In this way, all candidate regions in situations where there are no leakages are eliminated.

In the region growing algorithm, only a suitable threshold is chosen so that is grows to the appropriate leakage dimensions. Otherwise, in an extreme situation, the region could grow in such a way that it occupies the full extent of the image. The region is still dilated so that the contour serves as input for also a determination with the active contour algorithm, thus allowing a more accurate lesion area.

The value to be used for the contraction bias is very important for the performance of the method. It is intended to have a generative algorithm, invariant to several situations that may 
appear in the FA sequences. The issue of the unexpected variation of luminosity of the lesions seems to be well overcome by using a contour greater than the contour of the immediately after leakage and the monotony also seems to be guaranteed through the implemented method of analysis of the pixels along 5 images of the sequence.

As the algorithm has so many steps, problems that may appear can be resolved by following steps. For instance, filling vessel problems can be compensated by the mask dilation or the detection of candidates can be dissipated by the subtraction of images. However, the opposite may happen. A small problem can have a large effect on leakage segmentation. In brief, the pros and cons of each step should be predicted and try to balance them so that leakage segmentation is as accurate as possible.

As a summary, the steps that can affect the correct leak detection are mainly the registration, the vessel segmentation, the search for lesion candidates in the earliest image and the Otsu method. In turn, the search for lesion candidates in the earliest image, the inpainting vessels, the region growing algorithm and the active contour algorithm used may influence the correct delineation of leakage contours.

\subsection{Segmentation Results}

To evaluate the performance of the automatic segmentation of the CSC leaks, the results of the algorithm were compared with manual annotations. These were made by an ophthalmologist who is accustomated to performing FA exams. There are 3 training sequences with manual annotations from 5 possible sequences that have leaks.

As mentioned in section 3.2.10 there are some measures that can commonly be used to evaluate segmentation performance. In that section, the accuracy, the specificity, and the sensitivity were described. However, there are two other appropriate measures for this case. They range from 0 (no overlap) to 1 (complete congruence). The Jaccard index (JI) (equation 5.1) and the Dice similarity coefficient (DSC) (equation 5.2) are overlap ratio measures, that range from 0 (no overlap) to 1 (complete overlap).

$$
\begin{gathered}
J I=\frac{T P}{T P+F N+F P} \\
D S C=\frac{2 * T P}{2 * T P+F P+F N}
\end{gathered}
$$

In the present study, the true negative values are very high, since the correct segmentation of the lesion and the proposed segmentation have much lower area than the total content of the image. For this reason, accuracy and specificity values will naturally be high because they depend on the number of true negatives. On the other hand, the sensitivity, the Jaccard index and the Dice similarity coefficient are adequate for a good analysis of the performance of the algorithm and are calculated in this work because they do not consider the true negatives for 
the calculation of similarity. Jaccard is numerically more sensitive to mismatch when there is reasonably strong overlap. Dice values look nicer because they are higher for the same pair of segmentations, since the true positive values are multiplied twice in this coefficient. Table 5.1 shows the results of the performance measurements in the sequences that have manual annotations. The sequence $B$ and $G$ have a leakage occurrence, and the sequence $C$ has three leaks. Figure 5.7 shows the best and the worst results of the algorithm.

Although not indicated, there are frames in which the segmented regions by the proposed algorithm are all contained in the segmented region through manual annotations, so the SP reaches values of 1 . In this case, it can be concluded that the used contraction bias is in general very high. Also, the opposite happens, situations in which the sensitivity is 1 , so in these situations the proposed segmented region is greater than the real one. Indeed, there was great variation: sometimes the determined region is completely contained, sometimes it contains the true region. The $3^{\text {rd }}$ lesion of $C$ sequence is clearly a situation in which the region is determined in excess because it has high sensitivity values and a low standard deviation.

The borders of the lesions are not very well defined, they are blurred. The segmented region should include the transition zone with some angiographic haze, even if the transition zone has a little bit fluorescence than the background. The last frame of $G$ is a situation in which there is inadequate segmentation because the angiographic haze region is quite large and with different intensities whereby the region growing algorithm stops before the limit of the haze zone is reached. Figure 5.8 demonstrates the segmentation achieved, the manual annotation and the original image of the last frame of the $\mathrm{G}$ sequence.

Segmentations that have a small DSC and a small $\mathrm{Jl}$ are those in which the leaks present minor dimensions. In these cases, besides the lesions being of minor dimensions they also present a very low contrast with the background. The dye has not yet reached its maximum fluorescence, nor has the leak completely reached the subretinal space. As the intensity difference is very small between the lesion and the background, the active contour algorithm does not always adapt to the desired shape. Moreover, since the number of pixels in the region is small, with a little non-coincident region, the performance values decrease a lot.

The first leakage of $\mathrm{C}$ sequence has considerable dimensions but the segmentation performed is slightly different from reality because it systematically includes a region of high intensity close to the delimitation of the macula that the manual annotations do not include.

Since the standard deviations for some of the different performance metrics are high, then the performance metrics are widely spread. This means that the segmentations can be very close to the one desired in a given frame, as well as being away from the one desired in other frames. 
Table 5.1 - Performance of the train set images of 3 sequences.

\begin{tabular}{|c|c|c|c|c|c|c|c|c|c|c|c|}
\hline \multirow{2}{*}{ Sequence } & \multirow{2}{*}{ Condition } & \multirow{2}{*}{$\begin{array}{l}\text { Lesion } \\
\text { number }\end{array}$} & \multirow{2}{*}{ SN } & & $\begin{array}{c}\text { Annotated } \\
\text { leakage size }\end{array}$ & $\begin{array}{r}\text { Determined } \\
\text { leakage size }\end{array}$ & \multirow{2}{*}{ DSC } & \multirow{2}{*}{$\mathrm{Jl}$} & \multirow[b]{2}{*}{$\begin{array}{l}\text { Frame acquisition } \\
\text { time (minutes) }\end{array}$} & $\begin{array}{c}\text { Annotated } \\
\text { leakage size }\end{array}$ & $\begin{array}{l}\text { Determined } \\
\text { leakage size }\end{array}$ \\
\hline & & & & $\begin{array}{c}\text { Frame acquisition } \\
\text { time (minutes) }\end{array}$ & \multicolumn{2}{|c|}{ Pixels } & & & & \multicolumn{2}{|c|}{ Pixels } \\
\hline \multirow{3}{*}{ B } & Mean & \multirow{3}{*}{1} & $0.8341 \pm 0.1295$ & \multicolumn{3}{|c|}{ n.a } & $0.8644 \pm 0.1090$ & $0.7750 \pm 0.1571$ & \multicolumn{3}{|c|}{ n.a. } \\
\hline & Minimum & & 0.6385 & 1.72 & 1331 & 849 & 0.5845 & 0.4129 & 1.10 & 390 & 462 \\
\hline & Maximum & & 0.9632 & 5.43 & 5740 & 6064 & 0.9368 & 0.8811 & 5.43 & 5740 & 6064 \\
\hline \multirow{12}{*}{ c } & Mean & \multirow{3}{*}{1} & $0.8093 \pm 0.1175$ & \multicolumn{3}{|c|}{ n.a. } & $0.7150 \pm 0.1091$ & $0.5663 \pm 0.1264$ & \multicolumn{3}{|c|}{ n.a. } \\
\hline & Minimum & & 0.5953 & 3.39 & 8564 & 8467 & 0.4879 & 0.3227 & 0.69 & 110 & 263 \\
\hline & Maximum & & 0.9895 & 0.87 & 191 & 434 & 0.8616 & 0.7568 & 8.46 & 8102 & 8897 \\
\hline & Mean & \multirow{3}{*}{2} & $0.7649 \pm 0.1372$ & \multicolumn{3}{|c|}{ n.a. } & $0.7865 \pm 0.1005$ & $0.6599 \pm 0.1526$ & \multicolumn{3}{|c|}{ n.a. } \\
\hline & Minimum & & 0.5528 & 8.44 & 559 & 360 & 0.6725 & 0.5066 & 8.44 & 559 & 360 \\
\hline & Maximum & & 0.8418 & 0.87 & 316 & 354 & 0.8384 & 0.7218 & 1.07 & 360 & 358 \\
\hline & Mean & \multirow[b]{3}{*}{3} & $0.9504 \pm 0.0491$ & \multicolumn{3}{|c|}{ n.a. } & $0.8409 \pm 0.0881$ & $0.6948 \pm 0.1416$ & \multicolumn{3}{|c|}{ n.a. } \\
\hline & Minimum & & 0.8740 & 3.37 & 905 & 834 & 0.5673 & 0.3960 & 0.74 & 295 & 745 \\
\hline & Maximum & & 1 & $\begin{array}{c}0.93 \\
(0.74 \text { and } 0.87 \\
\text { too }) \\
\end{array}$ & 446 & 805 & 0.9097 & 0.8344 & 3.37 & 905 & 834 \\
\hline & Mean & \multirow{3}{*}{ All } & $0.8326 \pm 0.0977$ & \multicolumn{3}{|c|}{ n.a. } & $0.7458 \pm 0.1026$ & $0.6036 \pm 0.1184$ & \multicolumn{3}{|c|}{ n.a. } \\
\hline & Minimum & & 0.6513 & 3.39 & 4910 & 3514 & 0.4979 & 0.3227 & 0.69 & 110 & 263 \\
\hline & Maximum & & 0.9461 & 0.74 & 427 & 1034 & 0.8498 & 0.7388 & 8.46 & 8919 & 10091 \\
\hline \multirow[b]{3}{*}{ G } & Mean & \multirow[b]{3}{*}{1} & $0.8940 \pm 0.1201$ & \multicolumn{3}{|c|}{ n.a. } & $0.8407 \pm 0.1125$ & $0.7401 \pm 0.1636$ & \multicolumn{3}{|c|}{ n.a. } \\
\hline & Minimum & & 0.5418 & 9.63 & 1842 & 1002 & 0.5729 & 0.4014 & 1.67 & 228 & 568 \\
\hline & Maximum & & 1 & $\begin{array}{c}2.01 \\
(1.67 \text { and } 1.79 \\
\text { too })\end{array}$ & 330 & 605 & 0.9076 & 0.8308 & 3.86 & 744 & 803 \\
\hline \multirow{3}{*}{ All } & Mean & \multirow{3}{*}{ n.a. } & $0.8607 \pm 0.1167$ & & & & $0.8281 \pm 0.1073$ & $0.7179 \pm 0.1543$ & & & \\
\hline & Minimum & & 0.5418 & & n.a. & & 0.4879 & 0.3327 & & n.a. & \\
\hline & Maximum & & 1 & & & & 0.9368 & 0.8811 & & & \\
\hline
\end{tabular}


The results of the training sequences are considered to be good (with mean $\mathrm{Jl}$ being $0.7179 \pm 0.1543)$, although in either cases low performance values are obtained.

In addition to this validation, there were conversation with the ophthalmologist who confirmed that the training results obtained were quite good and according to what was intended. Detection in the training set occurred at $100 \%$.

For the training set, in addition to the sequences, whose results are presented in the table 5.1 , there were then two sequences that showed leakages from the beginning and were always detected and segmented ( $F$ and $S$ sequence), and there were two sequences that did not detected lesion because in fact, they did not exist ( $\mathrm{H}$ and $M$ sequence). An example of a noleaks sequence is shown in figure 5.9 .

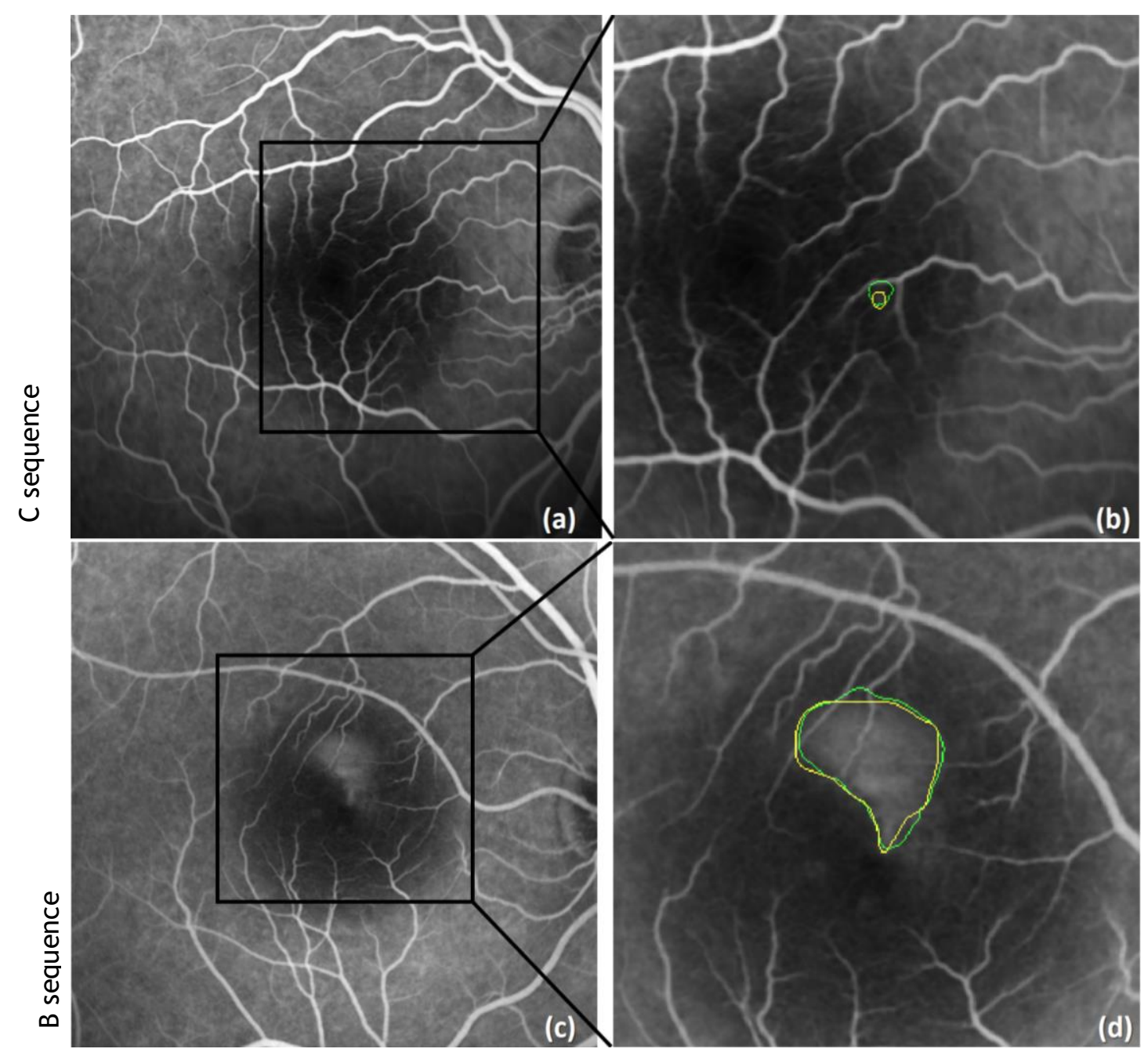

Figure 5.7 - Algorithm segmentation results: original images (a - c), worst segmentation (b) (yellow - manual annotation, green - achieved segmentation), best segmentation (d) (yellow manual annotation, green - achieved segmentation). 

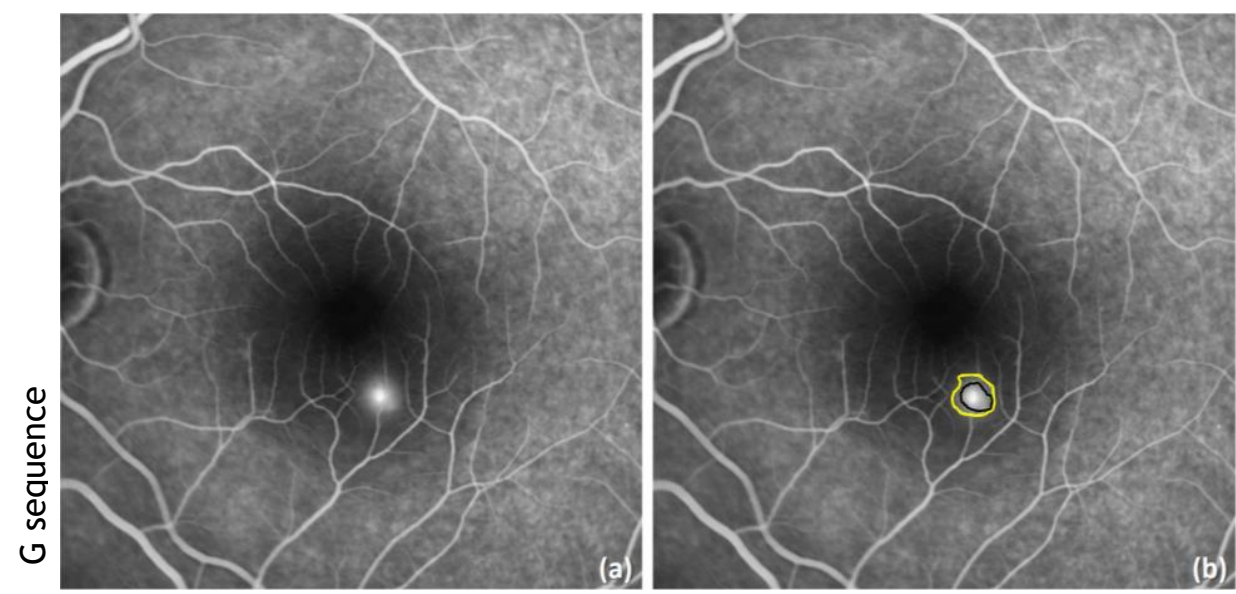

Figure 5.8 - Example of difficult determination of the region by presenting a haze region with large dimensions and different intensities: original images (a), original image with segmentation result (b) (yellow - manual annotation, black - achieved segmentation).
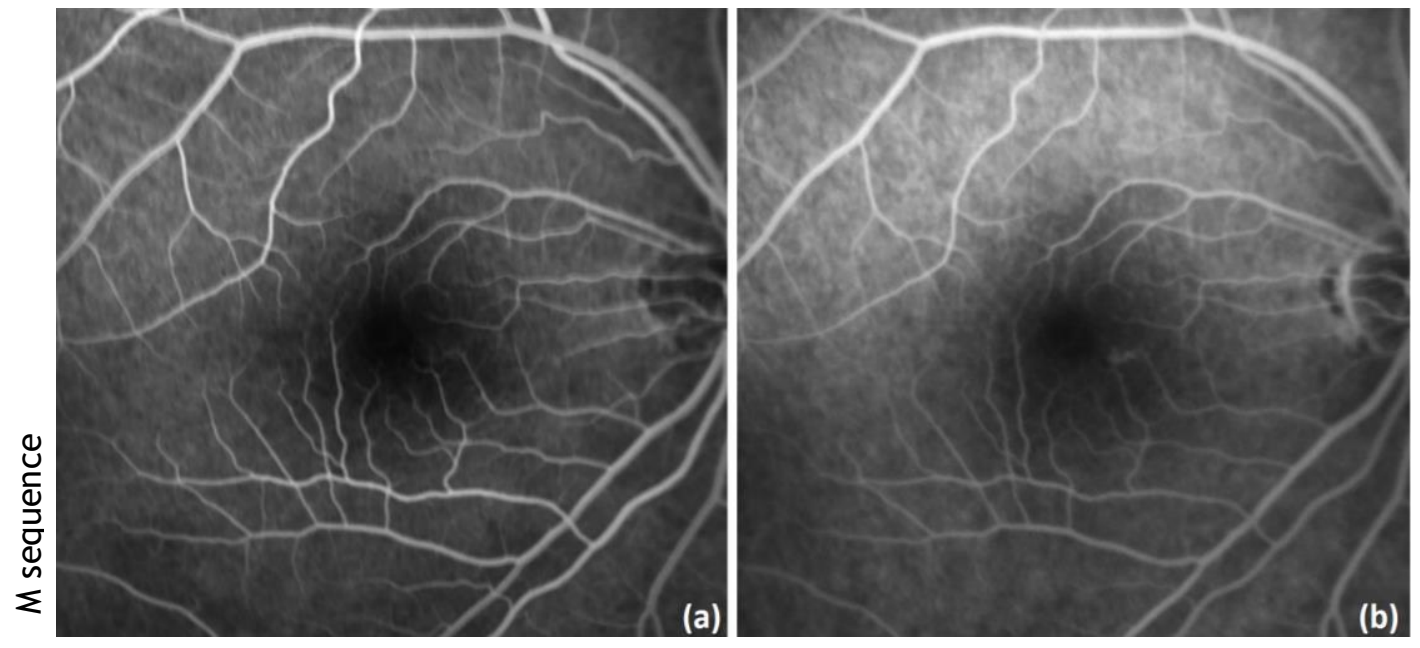

Figure 5.9 - Example of a sequence without a leakage: first frame (a), last frame (b).

The appointed explanations for a decrease in segmentation performance are: the difficulty of adapting the contour in situations of low contrast between the background and the leaks, the inpainting of the vessels partially change the contours of the leaks in the images where the segmentation is performed, and the difficulty to recover the contours of the images in case that there is considerable variation of luminosity in consecutive images of the sequence.

Manual annotations are a tedious process and are observer dependent but they provide a very safe approximation of the manual annotation. Minor distortions in the closure of the contours may have slightly lowered the performance values.

The algorithm was also experienced in the test set, but unfortunately it was not yet possible to collect all manual annotation for these cases. Only the worst cases were annotated and feedback was obtained for the detection of leakages in the sequence. Table 5.2 presents the performance metrics with the results obtained and for the existing manual annotations. 
The test set is slightly different from the type of images found in the training set. The test has strong luminosity effects, the lesions have very different intensity means, there are large time jumps between the acquired frames and the number of hyperfluorescence regions is considerable. As for the first two problems, it was expected that the intensity normalization method would solve them. For the last problem, it is only a problem in the definition of the contour. However, in very large time jumps instead of the active contour algorithm, the algorithm of region growing started to be used as can be seen in figure 4.24 because the active contours algorithm would have difficulty adapting to a region decrease so high.

Table 5.2 - Performance of the test set images that have manual annotations.

\begin{tabular}{|c|c|c|c|c|c|c|}
\hline \multirow{2}{*}{ Sequence } & $\begin{array}{c}\text { Frame } \\
\text { acquisition } \\
\text { time (min) }\end{array}$ & SN & DSC & JI & $\begin{array}{c}\text { Annotated } \\
\text { Leakage } \\
\text { Size }\end{array}$ & $\begin{array}{c}\text { Determined } \\
\text { Lesion Size }\end{array}$ \\
\cline { 3 - 7 } & 4.93 & 0.7950 & 0.8856 & 0.7947 & 7697 & 6122 \\
\hline \multirow{3}{*}{$\mathrm{D}$} & 5.67 & 0.7904 & 0.8748 & 0.7774 & 8418 & 6795 \\
\cline { 2 - 7 } & 7.11 & 0.6316 & 0.7742 & 0.6316 & 10813 & 6830 \\
\cline { 2 - 7 } & 10.30 & 0.5083 & 0.6740 & 0.5083 & 15755 & 8009 \\
\hline E & 9.96 & 0.7546 & 0.8538 & 0.7449 & 6064 & 4655 \\
\hline mean & n.a. & $0.6960 \pm 0.1240$ & $0.8125 \pm 0.0889$ & $0.6914 \pm 0.1204$ & \multicolumn{2}{|c|}{ n.a. } \\
\hline
\end{tabular}

Regarding the test set, the small and low intensity regions were not detected. The main reasons for failure were that the Otsu method had determined a higher threshold than intended. Since there were leakages of different intensities means, there were several peaks in the histogram, so the threshold determined by the Otsu method did not adequately separate leaks from the background. Small low intensity leaks also may not have a considerable peak in the histogram so the calculated threshold may not allow the inclusion of these region in the class belonging to the leaks. Also, the minimum conditions introduced by the Otsu method may also be overfitting with the training data and damaging the test results.

Regarding the $D$ test set segmentation and with the analysis of the table 5.2, it is indicated that the later the frame, the lower the value of the performance metric. The main reason is that the last frame did not grow enough because it is a very large region and with a wide range of intensities so the method of region growing failed to catch the entire leakage area. As the contours of the early frame leaks are determined, the active contour algorithm becomes closer to the one desired. Figure 5.10 is the last frame of the $D$ sequence that has only one handannotated leakage, two regions segmented by the proposed method in this dissertation and it is believed that there are still two lesions that were not detected.

The area of the largest leak of the $\mathrm{E}$ sequence do not overlap well with manual markings because as the leak has darker regions inside, the active contour algorithm tends to contract in such way that these regions are erroneously out of segmentation. Figure 5.11 has some images that allow to understand what happened in this situation. The darker regions are marked with orange in figure 5.11 (b). 
Although annotations are only available for the most deviant cases, the mean $\mathrm{Jl}$ in these cases was $0.6914 \pm 0.1204$.

In the appendix, the leaks segmentations achieved with the proposed method for the angiographic sequences of the used dataset are available.

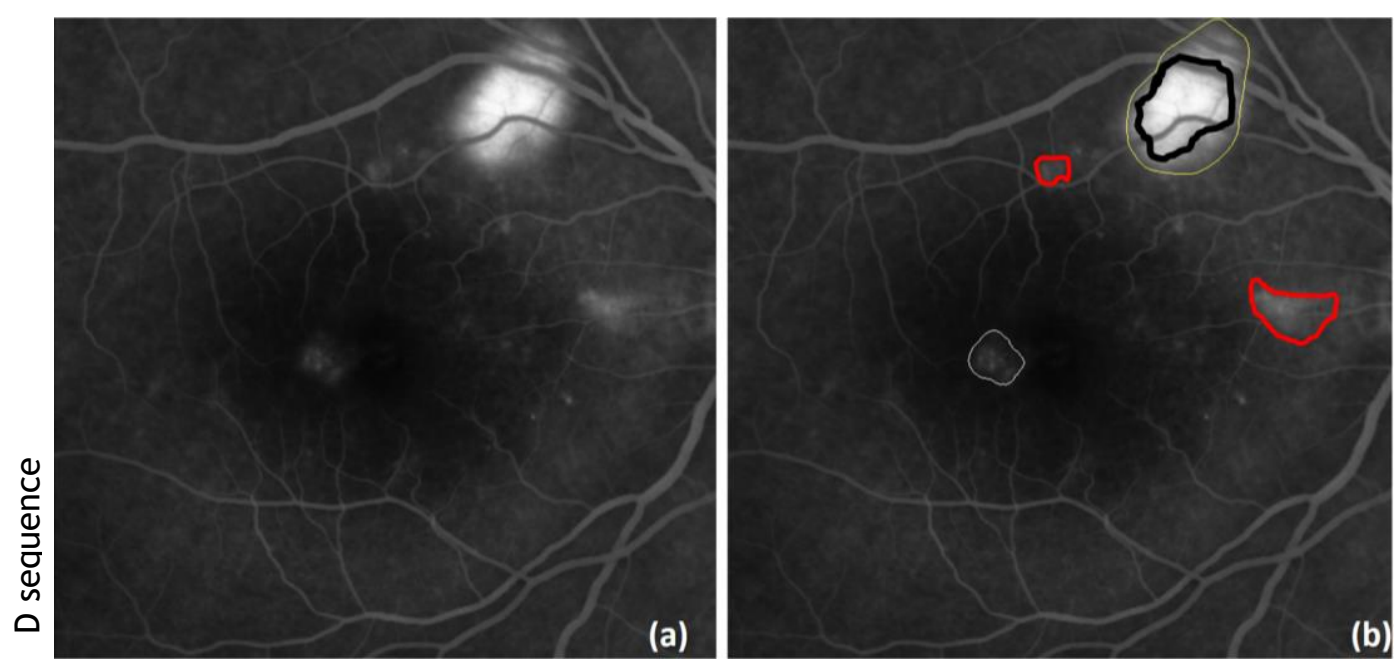

Figure 5.10 - Example of a test image: original image (a), original image with annotations (b) (yellow - manual annotation, black - estimated segmentation of the leakage 1, white estimated segmentation of the leakage 2 , red - possible missing leakage regions).

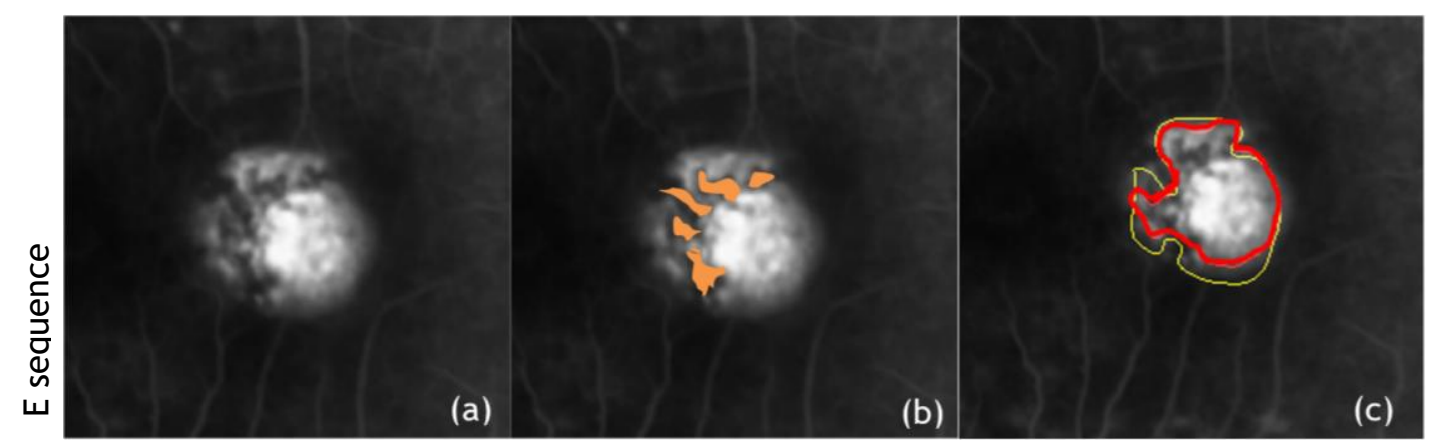

Figure 5.11 - Leakage with dark regions inside: original image (a), original image with the dark regions marked (b) and the leakage segmentation with the implemented method (red) and the manual annotation (yellow) (c).

\subsection{Size Progression of the Leakage}

The graph of size progression of the lesion allows to take some conclusions about the typology of the lesions. Firstly, it allows to know if the lesions are of an early initiation and remain with similar dimensions, or they are late occurrences and grow a lot. Second, when it is found that the area of segmented leakage does not grow over time, then fluorescein has already fully penetrated the region where the detachment occurred. Therefore, with the measured leakage 
area it is possible to predict the size of the detachment which is related to the severity of the disease.

The grow velocity graphs do not have much significance in this context, because as the distribution of images over time is not uniform, the graph would present values close to $0 \mathrm{~mm}^{2} / \mathrm{s}$ in images with very short acquisition intervals and excessively high values for very large acquisition intervals. To plot this type of graph must have a relatively constant acquisition interval.

In the representations of the size progression of the leakages in figure 5.12 , it is possible to note the leakages size can be very different. Furthermore, these types of representations appear to have two phases. A first of clear growth, and a second phase in which there is a stabilization of the size of the leak. The growth of leak in sequence $S$ is not very considerable after 5 minutes, which may mean that the fluid has completely attained the choroidal material in the detachment. The same happens with 2 leakages of the sequence $C$. The leaks that at the end of the examination have a smaller area, are also those that seem to achieve a stability of the dimensions earlier. This happen naturally because the same amount of fluorescein dye covers the entire detachment earlier for smaller lesions.

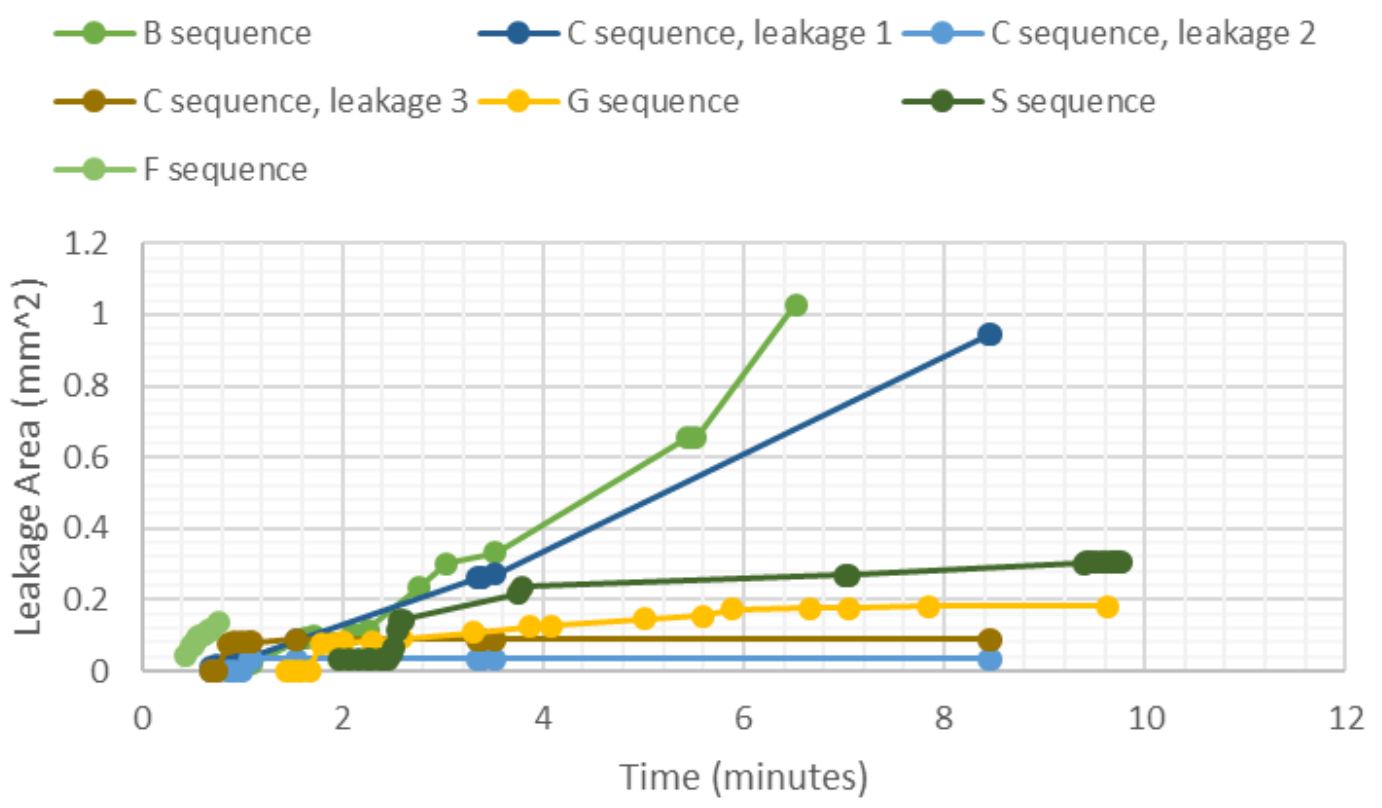

Figure $\mathbf{5 . 1 2}$ - Size progression of different leakages in different sequences.

\subsection{Intensity Progression of the Leakage}

The mean intensity of the leakage regions is, in turn, important in the analysis of the course of the FA exams. The intensity of the leaks was expected to increase gradually over time before the fluorescence loss. Then, it is possible to identify if the acquisition of the images happened 
at a time of loss of fluorescence. Furthermore, if there are problems with the image luminosity, this information can be deduced in a graph of the variation of the intensity of the lesions over time.

In figure 5.13, three examples of sequences are represented. There are clearly outdated peaks in the trend of increasing the intensity of lesion regions over time. These peaks correspond to abnormal variations in the luminosity of the image. However, it is not possible to notice a decrease in the intensity of the images and therefore the acquisition does not take place at a time of loss of fluorescence. The identification of images with considerable variations of luminosity and in which there is loss of fluorescence can be affected by the quality of the segmentations.

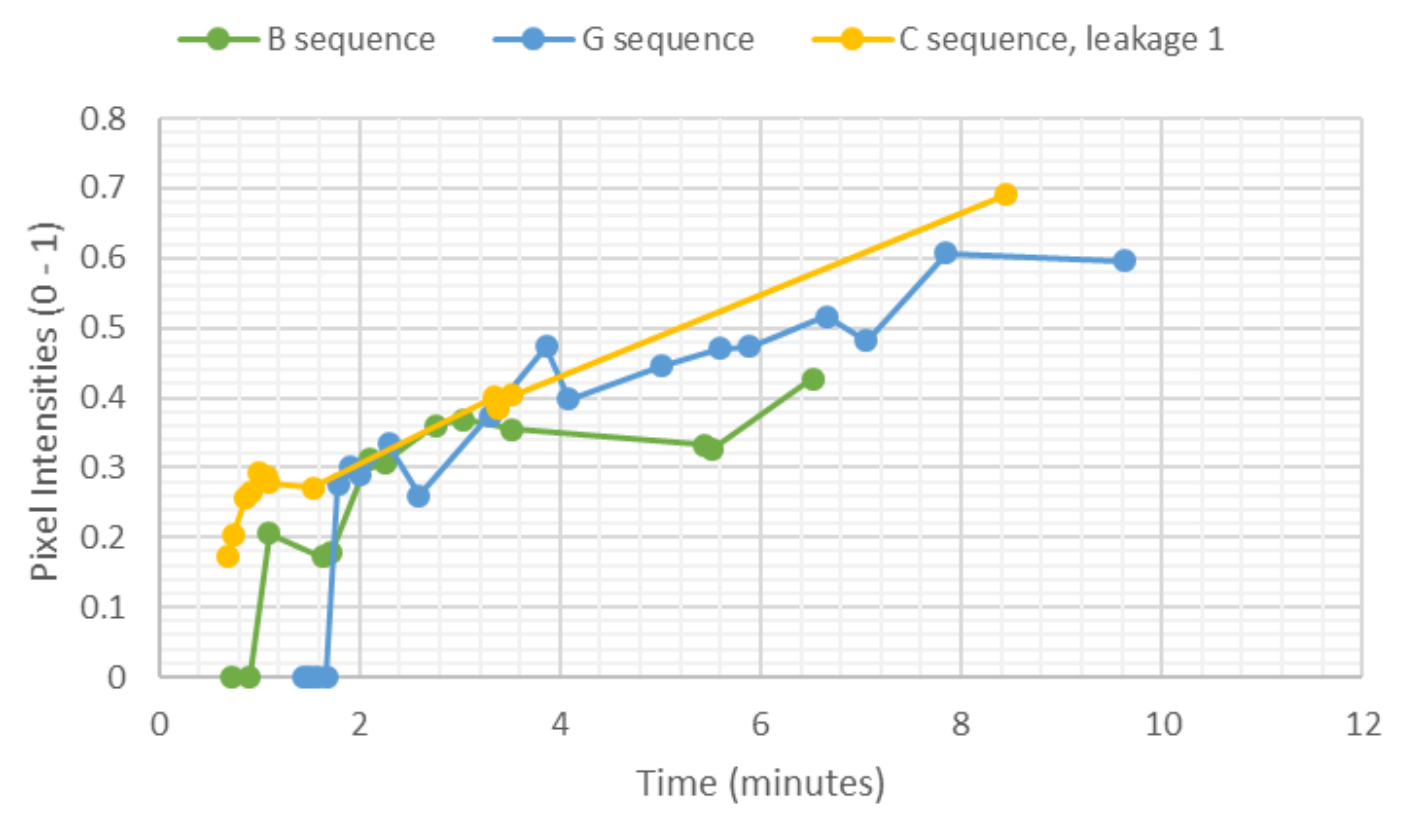

Figure 5.13 - Mean intensity progression of different leakages in different sequences.

\subsection{Computational Time of the Algorithm}

CAD tools save time and attention of health professionals in solving health problems. In this sense, the computational time of the algorithms developed to help in this purpose should be as fast as possible.

As the algorithm developed in this work is composed of many steps, it was evaluated the global computational time and the computational time of each step (table 5.3). For the calculation of these values, it was considered that the sequences are constituted in average by 15 images with one leakage. 
Table 5.3 - Computational time of the algorithm.

\begin{tabular}{|l|c|}
\cline { 2 - 2 } \multicolumn{1}{l|}{} & Time (seconds) \\
\hline Image reading & 1.4301 \\
\hline operations & 0.9380 \\
\hline Time recognition & 2.7122 \\
\hline Image denoising & 3.7169 \\
\hline Image registration & 3.2442 \\
\hline Vessel segmentation & 5.5455 \\
\hline Search for early leaks & 1.1158 \\
\hline Vessel inpainting & 0.1737 \\
\hline Optic disc detection & 5.8395 \\
\hline Intensity normalization & 0.0180 \\
\hline Background removal & 21.9716 \\
\hline Leakage segmentation & 46.7054 \\
\hline Global Time &
\end{tabular}

Although the algorithm has several steps, the global mean time seems acceptable. The step with the most time spent is the segmentation given that it is submitted to intermediate steps like the region growing segmentation and the active contour segmentation. The intensity normalization, the segmentation of blood vessels, the search for lesion candidates in the first frame and image registration are the steps following the segmentation that have a longer computational time.

The processor used was Intel (R) Core TM i7-5829K CPU @ $3.30 \mathrm{GHz}$, and the RAM was 32 GB. Computing times will naturally increase considerably on less performing processors and with less RAM. 


\section{Chapter 6}

\section{Conclusions and Future Work}

This thesis had two main objectives: the segmentation of the central serous chorioretinopathy leakages in FA images and the representation of the size progression of the leakage over time also in FA images. At the end, the objectives were fulfilled.

Indeed, central serous chorioretinopathy is a disease with a rather large prevalence. Since the algorithm allows the quantification of lesion area and then may predict the severity of visual loss, it probably may be useful to improve the treatment of the disease. No CAD tool was found for the CSC characterization in FA images and in the case of other similar leakage problems, the number of proposed methodologies is still very low. For the images of this work never before had been developed an algorithm to segment CSC leaks. The dataset is private and the images are fairly recent. There are no other approaches to this problem either with these images or with others so that the results cannot be compared. Even for other similar leaks in FA images, the segmentation is usually confined to a smaller region manually selected in the image and they have very low performances values. These methods are very dependent on the user's opinion to discern at the outset what is leakage and is therefore semi-automatic. This work is therefore a pioneer work in the way it tries to aid in the diagnosis of this disease.

A comparative method was the chosen to solve the problem. The comparison is achieved by subtracting early frames from the late frame by which it is possible to detect mainly the occurrence of changes over time. The dye leakage grows with the increase of time acquisition of the images, then it is an objective solution for leak detection.

Although subtraction is a good solution in the identification of regions that increase in intensity over time, it is also necessary to minimize the influence of elements that may hinder leakage segmentation. The vessels were segmented and the optic disc were detected because they have similar pixel intensities to the lesions. The background varies greatly in brightness so it was removed. The frames are also chosen, those in which the anatomical structures are correctly identifiable, i.e., the fluorescence has completely filled the vessels and has not yet 
lost fluorescence, and they need to be registered. By the indications received from an ophthalmologist, the detection works very well so these processing steps are appropriate.

After subtraction, the identification of candidates with the Otsu method is performed. The candidates undergo a region growing process in the image with the inpainted vessels and finally the active contour algorithm is applied to obtain the segmentation of the leaks in all images of the sequence. The results in the training set are good. In the test set, the images had very large luminosity effects, there are many leakages and they have different sizes and intensities. The results of segmentation were slightly worse and the detection failed in the smaller lesions and with little contrast with the background.

As a future work, the method of region growing should be adapted to solve the problems of the extended haze regions and regions with wide intensities distributions. Regarding the issue of detection, an alternative method to Otsu should be introduced in order to solve situations where there is some difference in intensity means of different lesions. Furthermore, an automatic method of selection of the early and frames should be introduced so that the segmentation results of the algorithm would not be dependent on the user's perception.

Although the dataset is very small, it is very diversified. It includes situation in which the optic disc is left and right, has situation without leakages, with one and with more than one leak, has situation of decrease and increase of the intensity of the images, there are situations in which the leakage appears initially and other not. With so many different situations, the algorithm is surely more robust in new images that can be provided. Moreover, it will be attempted to increase the number of manual annotations.

It is essential to have a defined protocol in the next acquisitions so that the algorithm can be adapted to the doctor's expectations, the leakage segmentation can be well defined and the maximum possible situations that can be occur in the images can be predicted. For instance, it is essential to have as many images as possible so that the graph of lesion growth progression can be traced over time, which is also one of the objective of the work. With more images, the active contours also adapt more easily since the transition between the regions considered correct is not so great.

A software application has been developed but needs to be upgraded. CAD tools are just a second opinion, power is always on the side of the doctors. As a future work, the application should have adding features such as changing the points of the contour of the segmented regions so that in the end the ideal segmentation is achieved.

Furthermore, a possible ambition is to test this algorithm in another type of disease such as macular edema. In this sense, the algorithm would be tested in public databases once used in the methods described in the state of the art.

Regarding the graphical representations developed, they seem to be useful. The size progression of the leak over time gives important information about the type of leak and it allows to identify the moment when the accumulation of fluorescein stagnates. The mean 
intensity progression of the leakage over time allows to analyze situations of strong variation of luminosity and to identify if the image acquisition happened at a moment in which the loss of fluorescence begins to be verified. 


\section{References}

[1] A. Siegel and H. Sapru, Essential Neuroscience, Baltimore, MD: Lippincott, Williams \& Wilkins, pp. 268-288, 2005.

[2] J. Marcuson and T. Riley, "Central serous chorioretinopathy," Optometry, vol. 79, pp. 241251, 2008.

[3] R. Liegl and M. W. Ulbig, "Central serous chorioretinopathy," Ophthalmologica, vol. 232, no. 2, pp. 65-76, 2014.

[4] M. B. Breukink et al., "Chronic central serous chorioretinopathy: long-term follow-up and vision-related quality of life," Clin. Ophthalmol., vol. 11, pp. 39-46, 2017.

[5] C. E. Riva, J. E. Grunwald, S. H. Sinclair and B. L. Petrig, "Blood velocity and volumetric flow rate in human retinal vessels," Invest. Ophthalmol. Vis. Sci., vol. 26, no. 8, pp. 11241132, 1985.

[6] C. P. Wilkinson, "Mysteries regarding the surgically reattached retina," Trans. Am. Ophthalmol. Soc., vol. 107, pp. 55-57, 2009.

[7] W. Tasman and E. A. Jaeger, The Wills Eye Hospital Atlas of Clinical Ophtalmology, $2^{\text {nd }}$ ed. Lippincott Williams \& Wilkins, pp. 168-175, 2001.

[8] D. Bujarborua, "Long-term follow-up of idiopathic central serous chorioretinopathy without laser," Acta Ophthalmol. Scand., vol. 79, no. 4, pp. 417-421, 2001.

[9] H. Rabbani, M. J. Allingham, P. S. Mettu, S. W. Cousins and S. Farsiu, "Fully automatic segmentation of fluorescein leakage in subjects with diabetic macular edema," Investig. Ophthalmol. Vis. Sci., vol. 56, no. 3, pp. 1482-1492, 2015.

[10] S. Veleri, C. H. Lazar, B. Chang, P. A. Sieving, E. Banin and A. Swaroop, "Biology and therapy of inherited retinal degenerative disease: insights from mouse models," Dis. Model. Mech., vol. 8, no. 2, pp. 109-129, 2015.

[11] D. L. Nickla and J. Wallman, "The multifunctional choroid," Prog. Retin. Eye Res., vol. 29, no. 2, pp. 144-168, 2010.

[12] L. Duan, Y.-J. Hong and Y. Yasuno, "Automated segmentation and characterization of choroidal vessels in high-penetration optical coherence tomography," Opt. Express, vol. 21, no. 13, pp. 15787-15808, 2013. 
[13] K. A. Toops, L. X. Tan and A. Lakkaraju, “A detailed three-step protocol for live imaging of intracellular traffic in polarized primary porcine RPE monolayers," Exp. Eye Res., vol. 124, pp. 74-85, 2014.

[14] P. Henkind and S. Hansen, "Ocular circulation," Physiology of the human eye and visual system, New York: Harper \& Row, pp. 98-155, 1979.

[15] S. A. Mehany, A. M. Shawkat, M. F. Sayed and K. M. Mourad, "Role of Avastin in management of central serous chorioretinopathy," Saudi J. Ophthalmol., vol. 24, no. 3, pp. 69-75, 2010.

[16] M. M. Teussink et al., "Oct angiography compared to fluorescein and indocyanine green angiography in chronic central serous chorioretinopathy," Investig. Ophthalmol. Vis. Sci., vol. 56, no. 9, pp. 5229-5237, 2015.

[17] P. Iacono, M. Battaglia Parodi, B. Falcomatà and F. Bandello, "Central Serous Chorioretinopathy Treatments: A Mini Review,” Ophthalmic Res., vol. 55, no. 2, pp. 76-83, 2015.

[18] R. F. Spaide, “Central serous chorioretinopathy," Medical Retina, Krieglstein GK, Weinreb RN (eds). Heidelberg, Springer, chap. 6, pp. 77-93, 2005.

[19] G. Borkhade and R. Raut, “Application of Neural Network for Diagnosing Eye Disease," Especial Issue of International Journal of Electronics Communications \& Soft Computing Science and Engineering, pp. 174-176, 2015.

[20] M. M. Shahin, "Angiographic characteristics of central serous chorioretinopathy in an Egyptian population,” Int. J. Ophthalmol., vol. 6, no. 3, pp. 342-345, 2013.

[21] J. David, A. S. Kumar and V. Viji, "Tracing of Central Serous Retinopathy from Retinal Fundus Images," IFMBE Proc., vol. 23, no. 1, pp. 331-334, 2009.

[22] N. Kitaya et al., "Features of abnormal choroidal circulation in central serous chorioretinopathy," Br. J. Ophthalmol., vol. 87, no. 6, pp. 709-712, 2003.

[23] V. Manjunath, M. Taha, J. G. Fujimoto and J. S. Duker, "Choroidal thickness in normal eyes measured using Cirrus UD optical coherence tomography,” Am. J. Ophtalmol., vol 150, no. 3, pp. 325-329, Sep. 2010.

[24] Y. Zhao, I. J. MacCormick, D. G. Parry, N. A. Beare, S. P. Harding and Y. Zheng, “Automated Detection of Vessel Abnormalities on Fluorescein Angiogram in Malarial Retinopathy," Sci. Rep., vol. 5, no. April, pp. 1-12, 2015.

[25] Y. Zhao et al., "Intensity and Compactness Enabled Saliency Estimation for Leakage Detection,” IEEE Trans. Med. Imaging, vol. 62, no. c, pp. 1-13, 2016.

[26] M. S. El-Shahawy, A. Elantably, N. Fawzy, K. Samir, M. Hunter and A. S. Fahmy, "Segmentation of Diabetic Macular Edema in fluorescein angiograms," Proc. - Int. Symp. Biomed. Imaging, pp. 661-664, 2011.

[27] A. S. Fahmy, W. M. Abdelmoula, A. E. Mahfouz and S. M. Shah, "Segmentation of Choroidal Neovascularization lesions in fluorescein angiograms using parametric modeling of the intensity variation," Proc. - Int. Symp. Biomed. Imaging, pp. 665-668, 2011.

[28] C. L. Tsai, Y. L. Yang, S. J. Chen, C. H. Chan and W. Y. Lin, “Automatic characterization and segmentation of classic choroidal neovascularization using Adaboost for supervised learning," IEEE Nucl. Sci. Symp. Conf. Rec., pp. 3610-3612, 2010.

[29] H. Bay, T. Tuytelaars and L. Van Gool, “SURF: Speeded up robust features," Lect. Notes Comput. Sci. (including Subser. Lect. Notes Artif. Intell. Lect. Notes Bioinformatics), vol. 3951 LNCS, pp. 404-417, 2006. 
[30] G. Bebis et al., Advances in Visual Computing, Second International Symposium, Part II, p. 538, 2006

[31] C. L. Tsai, C. Y. Li, G. Yang and K. S. Lin, "The edge-driven dual-bootstrap iterative closest point algorithm for registration of multimodal fluorescein angiogram sequence," IEEE Trans. Med. Imaging, vol. 29, no. 3, pp. 636-649, 2010.

[32] R. Estrada, C. Tomasi, M. T. Cabrera, D. K. Wallace, S. F. Freedman and S. Farsiu, "Exploratory Dijkstra forest based automatic vessel segmentation: applications in video indirect ophthalmoscopy (VIO)," Biomed. Opt. Express, vol. 3, no. 2, pp. 327-339, 2012.

[33] T. F. Chan, B. Y. Sandberg and L. A. Vese, "Active Contours without Edges for VectorValued Images," J. Vis. Commun. Image Represent., vol. 11, no. 2, pp. 130-141, 2000.

[34] Y. Zhao, L. Rada, K. Chen, S. Harding and Y. Zheng, "Automated Vessel Segmentation Using Infinite Perimeter Active Contour Model with Hybrid Region Information with Application to Retina Images," IEEE Trans. Med. Imaging, vol. 62, no. c, pp. 1797-1807, 2015.

[35] A. M. Mendonça, B. Dashtbozorg, and A. Campilho, "Segmentation of the vascular network of the retina", Image Analysis and Modeling in Opthalmology, pages 85-109. CRC Press, 2014.

[36] S. Wang, Y. Yin, G. Cao, B. Wei, Y. Zheng and G. Yang, "Hierarchical retinal blood vessel segmentation based on feature and ensemble learning," Neurocomputing, vol. 149, no. PB, pp. 708-717, 2015.

[37] Q. Li, J. You, L. Zhang, D. Zhang and P. Bhattacharya, "A New Approach to Automated Retinal Vessel Segmentation Using Multiscale Analysis," Pattern Recognition, 2006. ICPR 2006. 18th Int. Conf., vol. 4, pp. 77-80, 2006.

[38] A. Aquino, M. E. Gegúndez-Arias and D. Marín, "Detecting the Optic Disc Boundary in Digital Fundus Feature Extraction Techniques," IEEE Trans. Med. Imaging, vol. 29, no. 11, pp. 1860-1869, 2010.

[39] J. Cheng et al., "Superpixel classification based optic cup segmentation," Lect. Notes Comput. Sci. (including Subser. Lect. Notes Artif. Intell. Lect. Notes Bioinformatics), vol. 8151 LNCS, no. PART 3, pp. 421-428, 2013.

[40] C. T. Kelley, "Iterative Methods for Optimization," Siam, pp. 56-58, 1999.

[41] Z. Rahman, D. J. Jobson and G. A. Woodell, "Retinex processing for automatic image enhancement," J. Electron. Imaging, vol. 13, no. 1, pp. 100, 2004.

[42] Z. K. Huang and K. W. Chau, "A new image thresholding method based on Gaussian mixture model," Appl. Math. Comput., vol. 205, no. 2, pp. 899-907, 2008.

[43] C. A. Lupascu, D. Tegolo and E. Trucco, "FABC: Retinal Vessel Segmentation Using AdaBoost," IEEE Trans. Inf. Technol. Biomed., vol. 14, no. 5, pp. 1267-1274, 2010.

[44] M. MeilPa and J. Shi, "Learning segmentation by random walks," Neural Inf. Process. Syst., pp. 1-7, 2001.

[45] R. Smith, "An Overview of the Tesseract OCR Engine", Ninth International Conference on Document Analysis and Recognition, vol. 2, pp. 629-633, 2007.

[46] R. Smith, "Hybrid Page Layout Analysis via Tab-Stop Detection", Tenth International Conference on Document Analysis and Recognition", pp. 241-245, 2009.

[47] T. Esteves, P. Quelhas, A. M. Mendonça, and A. Campilho, "Cell nuclei and cytoplasm joint segmentation using Sliding Band Filter", IEEE Transactions on Medical Imaging, vol. 29, no. 8, pp. 1463-1473, 2010. 
[48] S. Berchtold, C. Böhm, D. A. Keim, and H. P. Kriegel, "A cost model for model for nearest neighbor search in high-dimensional data space, $16^{\text {th }}$ ACM SIGACT-SIGMOD-SIGART Symposium on PODS, pp. 78-86,1997.

[49] V. Argyriou, J. M. Rincon, B. Villarini and A. Roche, Image, Video \& 3D Data Registration, Wiley, pp. 163, 2015.

[50] R. Pires, H. F. Jelinek, J. Wainer, E. Valle, and A. Rocha, "Advancing bag-of-visual-words representations for lesion classification in retinal images," PLoS One, vol. 9, no. 6, pp. 112, 2014.

[51] J. Svab, T. Krajnk, J. Faigl and L. Preucil, "FPGA Based Speeded Up Robust Features," 2009 IEEE International Conference on Technologies for Practical Robot Applications, pp. 35-41, 2009.

[52] A. M. M. Madbouly, M. Wafy and M. M. Mostafa, "Performane Assessment of Feature Detector-Descriptor Combination," IJCSI International Journal of Computer Science Issues, vol. 12, pp. 87-94, 2015.

[53] T. B. Terriberry, L. M. French and J. Helmsen, "GPU Accelerating Speeded-Up Robust Features," Conf. on Technologies for Practical Robot Applications, IEEE, pp. 35-41, 2009.

[54] R. Subbarao, "Robust Statistics Over Riemannian Manifolds for Computer Vision," M.S. thesis, Graduate School - New Brunswick Rutgers, New Jersey, pp. 66-68, 2008.

[55] R. Raguram, J. Frahm and M. Pollefeys, "A comparative analysis of RANSAC Techniques leading to adaptive real-time random sample consensus," Computer Vision, vol. 5303, pp. 500-513, 2008.

[56] Y. Chi, J. Ho and M. Yang, "A Direct Method for Estimating Planar Projective Transform," Computer Vision - ACCV 2010, $10^{\text {th }}$ Asian Conference on Computer Vision, Part II, pp. 268281, 2011.

[57] W. R. Chum, T. Hartkens and D. L. G. Hill, "Non-rigid image registration: theory and practice," The British Journal of Radiology, 77, pp. 140-153, 2004.

[58] B. Dashtbozorg, A. M. Mendonça, and A. Campilho, "Optic disc segmentation using the sliding band fi lter," Comput. Biol. Med., vol. 56, pp. 1-12, 2015.

[59] L. Lam, S. Lee, and C. Suen, "Thinning Methodologies - A comprehensive Survey", IEEE Transactions on Pattern Analysis and Machine Intelligence”, vol. 14, no. 9, pp. 870, 1992.

[60] A. Gupta, A. Cecen, S. Goyal, A. K. Singh, and S. R. Kalidindi, "ScienceDirect Structure property linkages using a data science approach: Application to a non-metallic inclusion / steel composite system," ACTA Mater., vol. 91, pp. 239-254, 2015.

[61] N. Otsu, "A Threshold Selection Method from Gray-Level Histograms", IEEE Transactions on Systems, Man and Cybernetics, vol. 9, no. 1, pp. 62-66, 1979.

[62] T. F. Chan, and L. A. Vese, "Active contours without edges", IEEE Transactions on Image Processing, vol. 10, no. 1, pp. 266-277, 2001. 


\section{Appendix}

\section{Complementary Results}

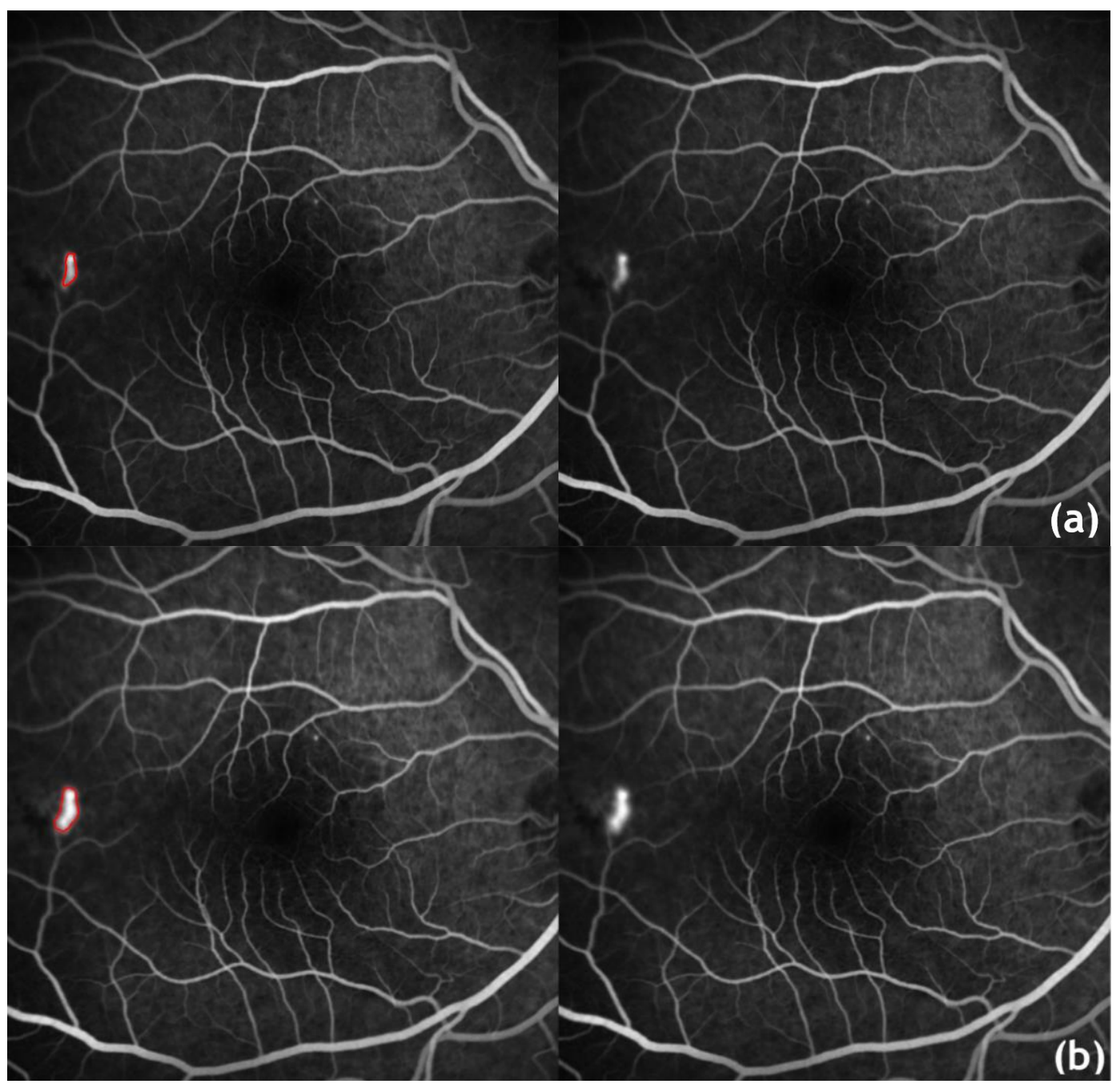

Figure A.1 - F sequence: original image with the segmentation achieved (in red) (left) and without (right); early frame (a), late frame (b). 


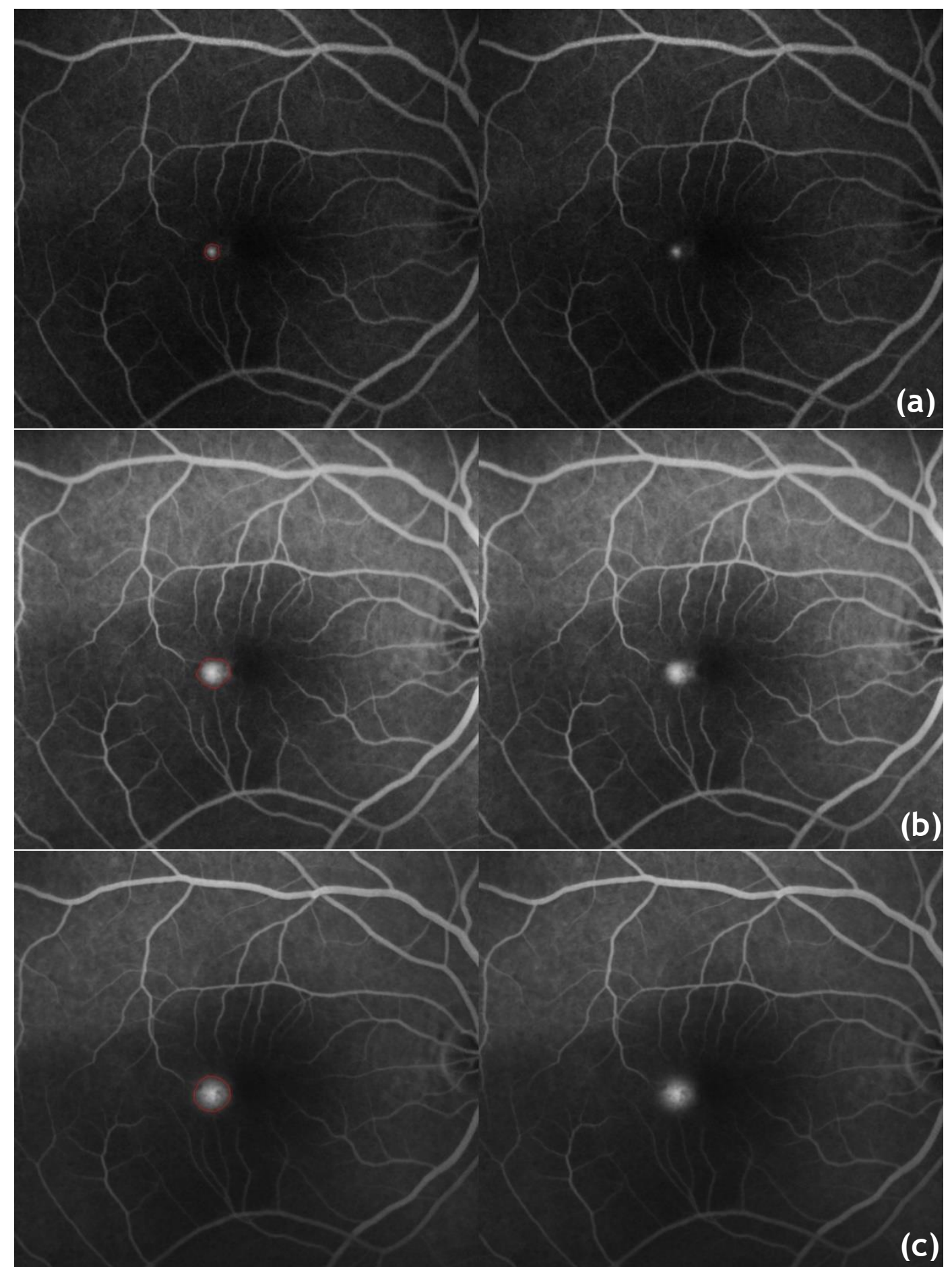

Figure A.2 - S sequence: original image with the segmentation achieved (in red) (left) and without (right); early frame (a), mid-time frame (b) and late frame (c). 


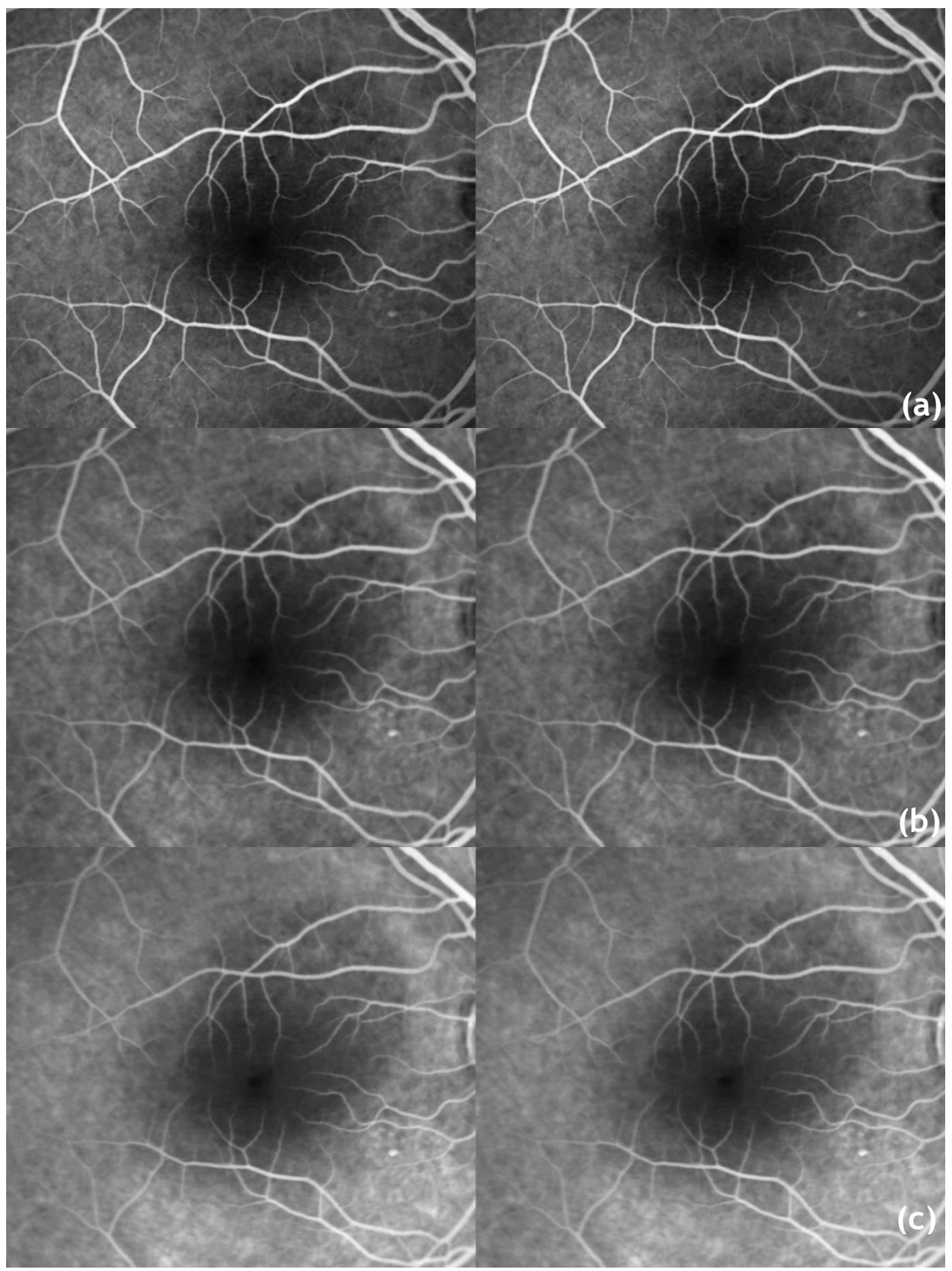

Figure A.3 - H sequence: original image with the segmentation achieved (in red) (left) and without (right); early frame (a), mid-time frame (b) and late frame (c).

As no leakage was found the left and right images are the same. 


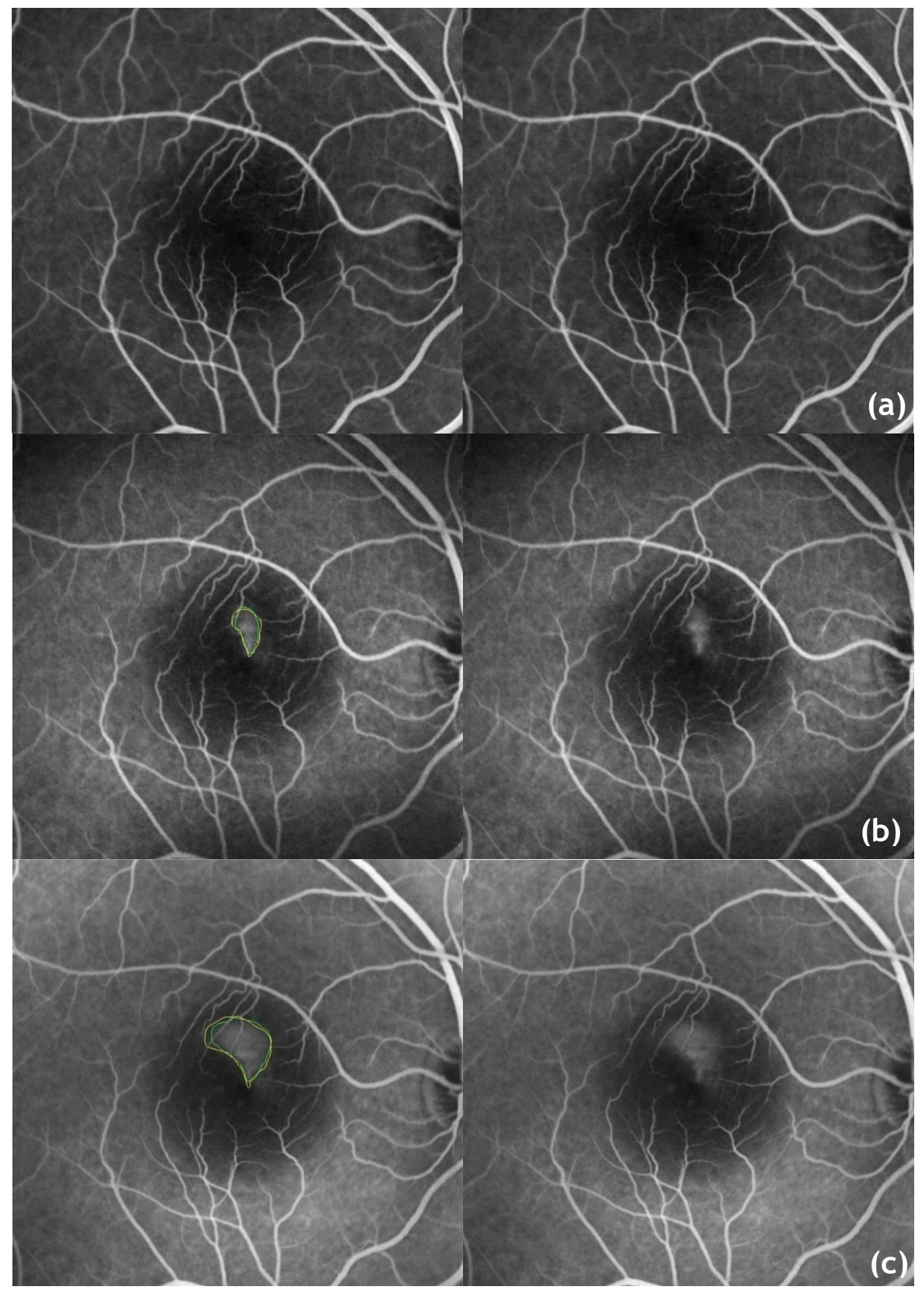

Figure A.4 - B sequence: original image with the segmentation achieved (in green) and annotated manually (in yellow) (left), and without (right); early frame (a), mid-time frame (b) and late frame (c). 


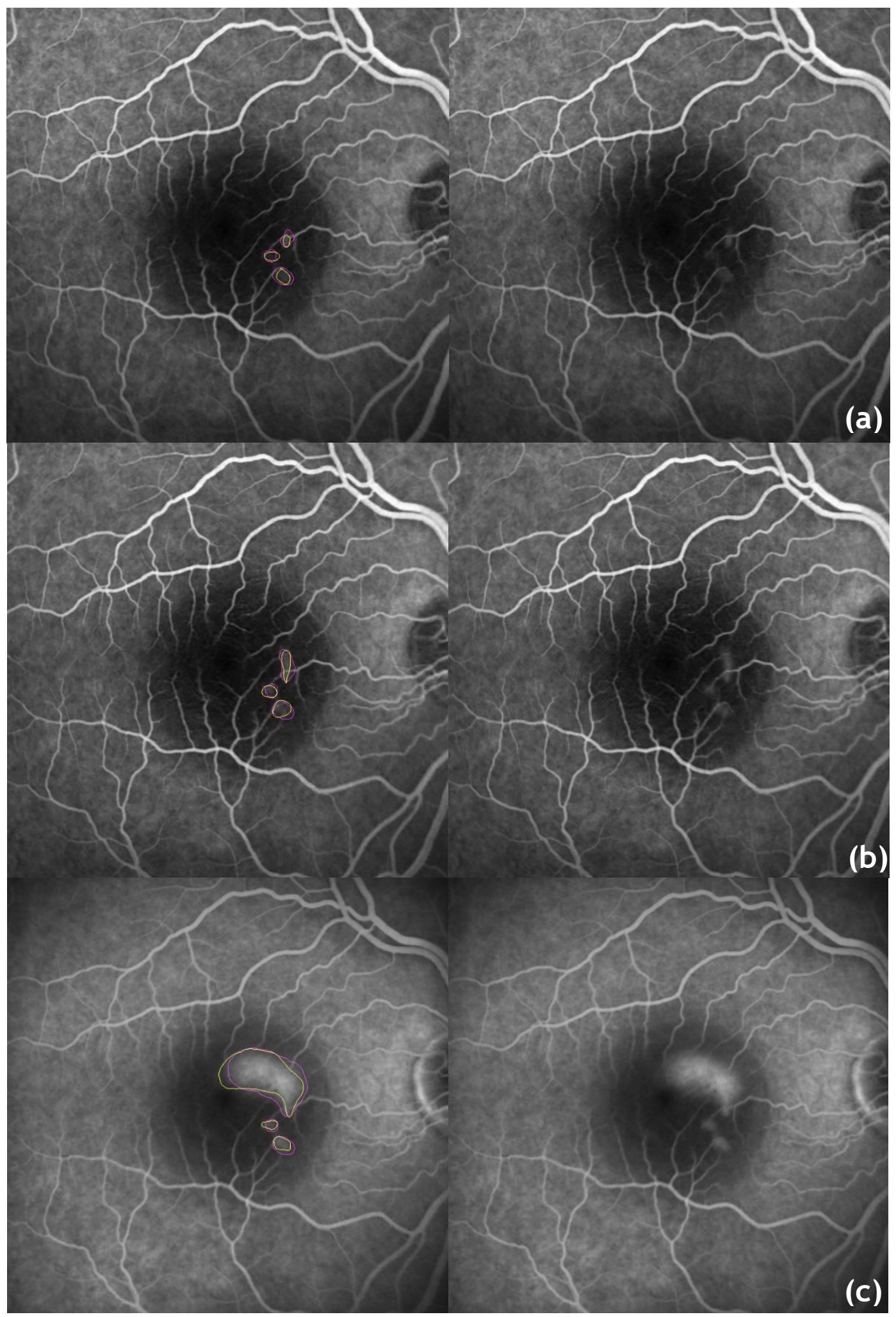

Figure A.5 - C sequence: original image with the segmentation achieved (in purple) and annotated manually (in yellow) (left), and without (right); early frame (a), mid-time frame (b) and late frame (c). 


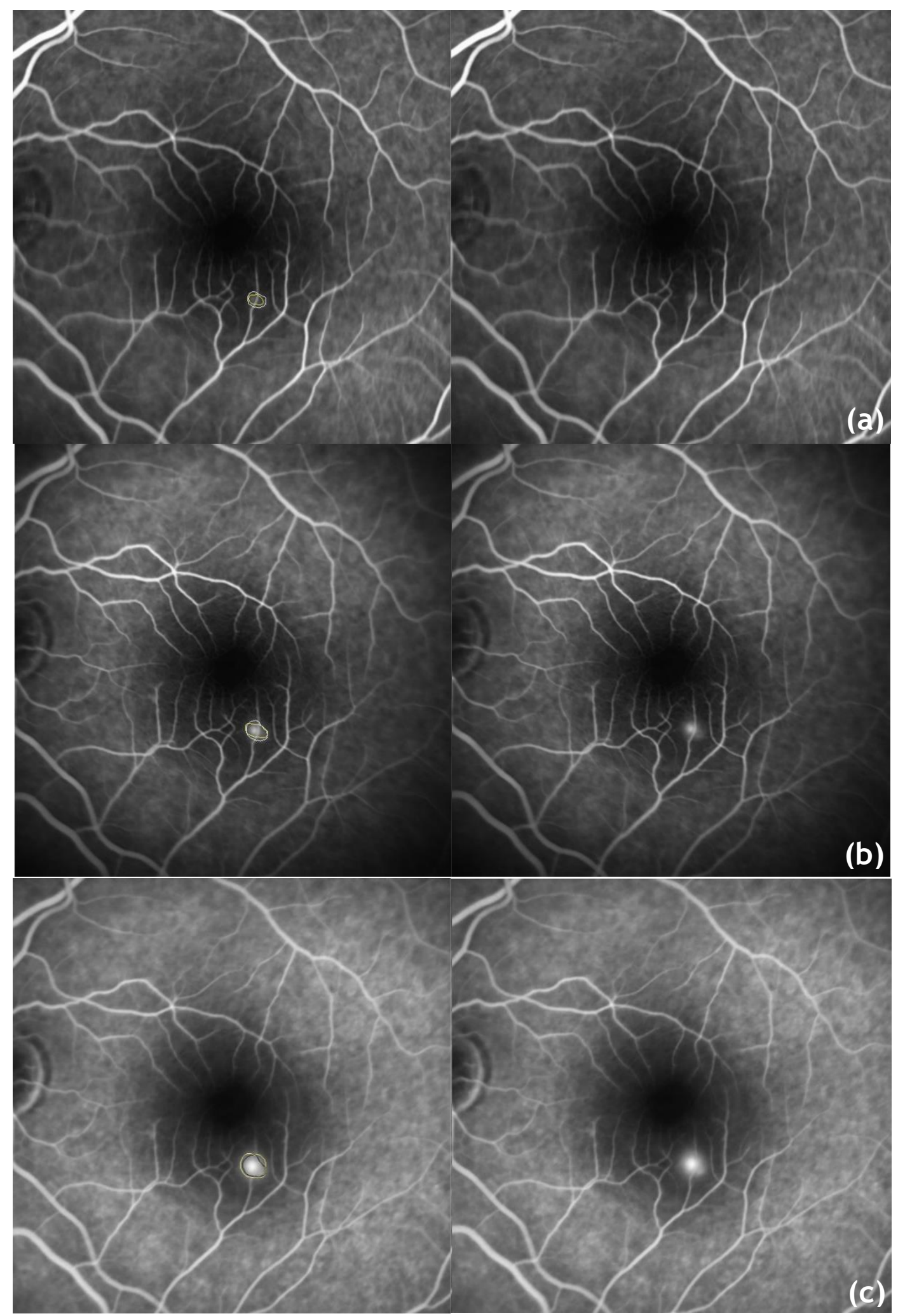

Figure A.6 -G sequence: original image with the segmentation achieved (with white in (a) and (b), and black in (c)) and annotated manually (in yellow) (left), and without (right); early frame (a), mid-time frame (b) and late frame (c). 


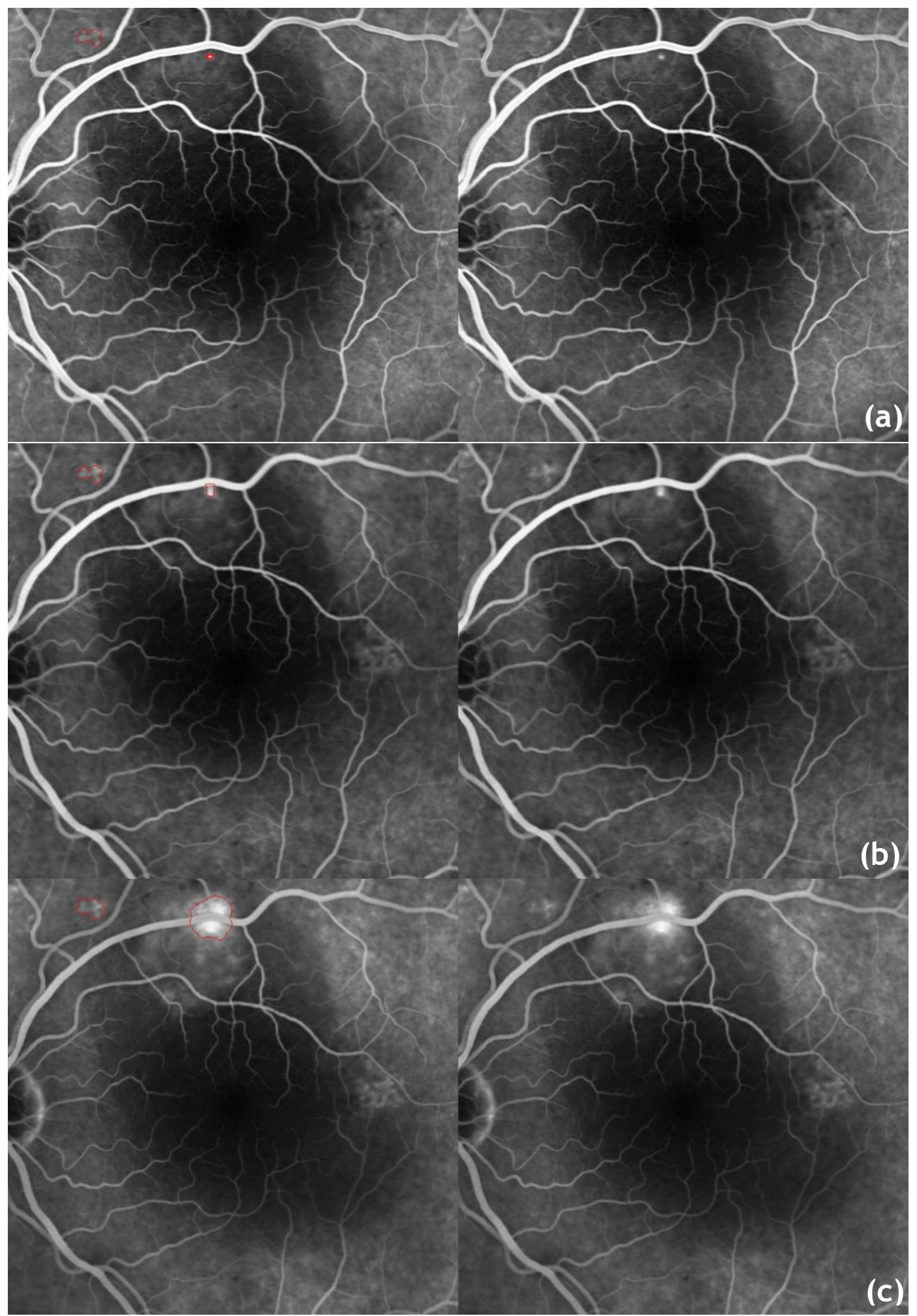

Figure A.7 - A sequence: original image with the segmentation achieved (in red) (left) and without (right); early frame (a), mid-time frame (b) and late frame (c). 


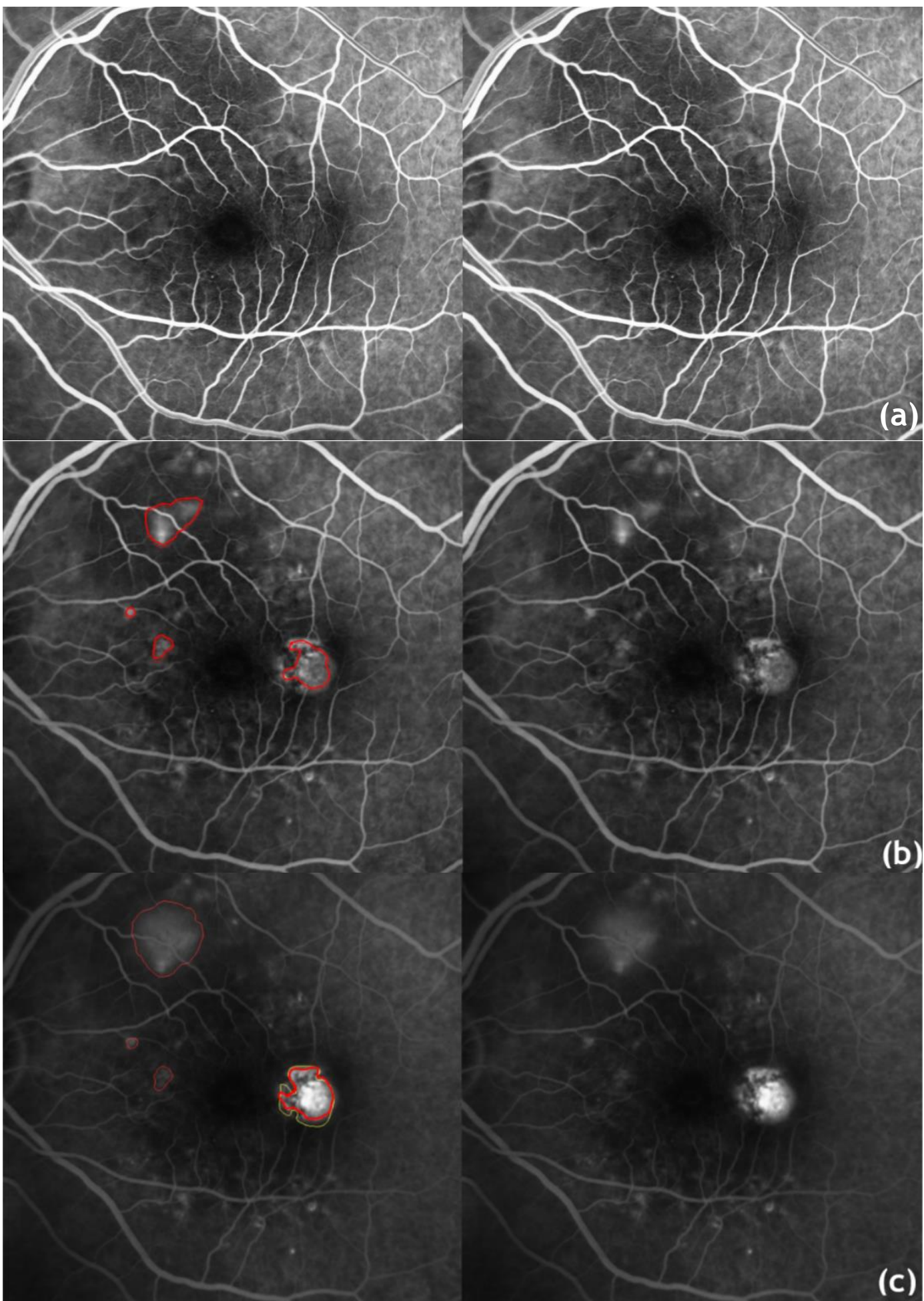

Figure A.8 - E sequence: original image with the segmentation achieved (in red) and annotated manually (in yellow) (left), and without (right); early frame (a), mid-time frame (b) and late frame (c).

Only the leakage of the last image of the sequence that was more deviated from reality was annotated manually. 

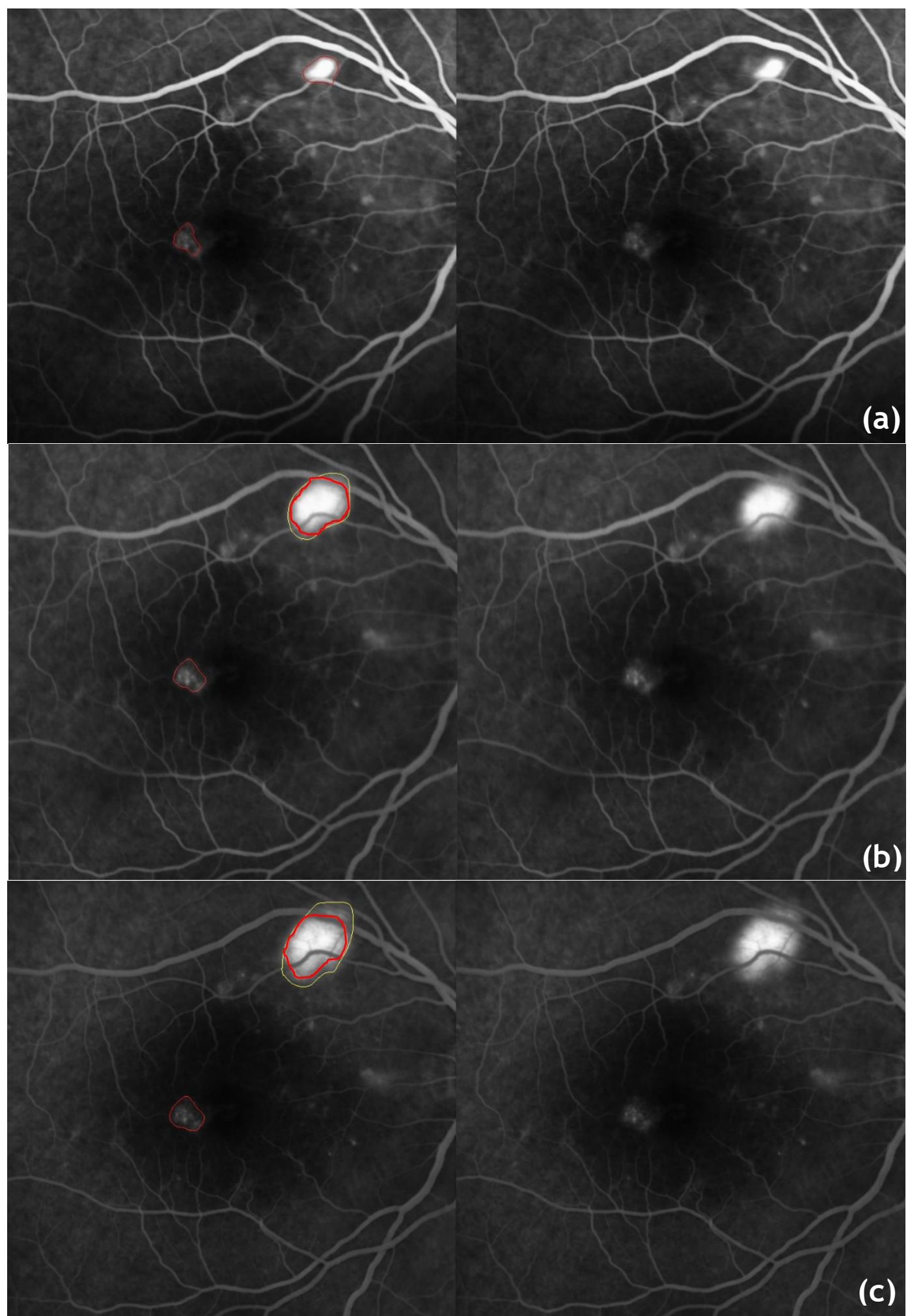

Figure A.9 - D sequence: original image with the segmentation achieved (in red) and annotated manually (in yellow) (left), and without (right); early frame (a), mid-time frame (b) and late frame (c). 